\title{
Hfq CLASH uncovers sRNA-target interaction networks involved in adaptation to nutrient availability
}

\begin{abstract}
Ira A. Iosub ${ }^{1}$, Marta Marchioretto ${ }^{2}$, Brandon Sy ${ }^{4}$, Stuart McKellar ${ }^{1}$, Karen J. Nieken ${ }^{3}$, Rob W.
\end{abstract} van Nues ${ }^{3}$, Jai J. Tree ${ }^{4}$, Gabriella Viero ${ }^{2}$ and Sander Granneman ${ }^{1 *}$

\section{Affiliations:}

${ }^{1}$ Centre for Synthetic and Systems Biology, University of Edinburgh, Edinburgh EH9 3BF, UK.

${ }^{2}$ Institute of Biophysics, CNR Unit at Trento, Italy.

${ }^{3}$ Institute of Cell Biology, University of Edinburgh, Edinburgh EH9 3FF, UK.

${ }^{4}$ School of Biotechnology and Biomolecular Sciences, University of New South Wales, Sydney 2052, NSW, Australia.

*To whom correspondence should be addressed:

Sander Granneman

e-mail: sgrannem@ed.ac.uk

Tel: +441316519082 


\section{Abstract}

By shaping gene expression profiles, small RNAs (sRNAs) enable bacteria to very

29 efficiently adapt to constant changes in their environment. To better understand how

30 Escherichia coli acclimatizes to changes in nutrient availability, we performed UV cross-

31 linking, ligation and sequencing of hybrids (CLASH) to uncover sRNA-target interactions.

32 Strikingly, we uncovered hundreds of novel Hfq-mediated sRNA-target interactions at specific

33 growth stages, involving many novel 3'UTR-derived sRNAs and a plethora of sRNA-sRNA

34 interactions. We discovered sRNA-target interaction networks that play a role in adaptation to

35 changes in nutrient availability. We characterized a novel 3'UTR-derived SRNA (MdoR), which

36 is part of a regulatory cascade that enhances maltose uptake by (a) inactivating repressive

37 pathways that block the accumulation of maltose transporters and (b) by reducing the flux of

38 general porins to the outer membrane. Our work provides striking examples of how bacteria

39 utilize sRNAs to integrate multiple regulatory pathways to enhance nutrient stress adaptation. 
Microorganisms are renowned for their ability to adapt to environmental changes by rapidly rewiring their gene expression program. These responses are mediated through integrated transcriptional and post-transcriptional networks. Control at the transcriptional level dictates which genes are expressed (Balleza et al., 2009; Martínez-Antonio et al., 2008) and is well-characterised in Escherichia coli. Post-transcriptional regulation is key for controlling adaptive responses. By using riboregulators and RNA-binding proteins (RBPs), cells can efficiently integrate multiple pathways and incorporate additional signals into regulatory circuits. E. coli employs many post-transcriptional regulators, including small regulatory RNAs (sRNAs (Waters and Storz, 2009)), cis-acting RNAs (Kortmann and Narberhaus, 2012), and RNA binding proteins (RBPs) (Holmqvist and Vogel, 2018). The sRNAs are the largest class of bacterial regulators, which work in tandem with RBPs to regulate their RNA targets (Storz et al., 2011; Waters and Storz, 2009). The base-pairing interactions are often mediated by RNA chaperones such as Hfq and ProQ, which help to anneal or stabilize the sRNA and sRNA-target duplex (Smirnov et al., 2017, 2016; Updegrove et al., 2016). Small RNAs can repress or stimulate translation and transcription, as well as control mRNA stability (Sedlyarova et al., 2016; Updegrove et al., 2016; Vogel and Luisi, 2011; Waters and Storz, 2009).

During growth in rich media, E. coli are exposed to continuously changing conditions, such as fluctuations in nutrient availability, $\mathrm{pH}$ and osmolarity. Consequently, E. coli elicit complex responses that result in physiological and behavioural changes such as envelope composition remodelling, quorum sensing, nutrient scavenging, swarming and biofilm formation. Even subtle changes in the growth conditions can trigger rapid adaptive responses. Accordingly, each stage of the growth curve is characterised by different physiological states driven by activation of different transcriptional and post-transcriptional networks. Moreover, growth phase dependency of virulence and pathogenic behaviour has been demonstrated in both Gram-positive and Gram-negative bacteria. In some cases a particular growth stage is non-permissive for the induction of virulence (Mäder et al., 2016; Mouali et al., 2018). Although the exponential and stationary phases have been characterised in detail(Navarro Llorens et al., 2010; Pletnev et al., 2015), little is known about the transition between these two phases. During this transition, the cell population starts to scavenge alternative carbon sources, which requires rapid remodelling of their transcriptome (Baev et al., 2006a, 2006b; Sezonov et al., 2007).

To understand sRNA-mediated adaptive responses, detailed knowledge of the underlying post-transcriptional circuits is required. In E. coli, hundreds of sRNAs have been discovered, but only a fraction of these have been characterised. A key step to unravel the roles of sRNAs in regulating adaptive responses is to identify their targets. To tackle this 
sRNA-target interactions, many more than previously anticipated (Han et al., 2016; Hör et al., 2018; Hör and Vogel, 2017; Lalaouna et al., 2015; Melamed et al., 2016; Waters et al., 2016).

To uncover sRNA-target RNA interaction dynamics that take place during the entry into stationary phase, we applied UV cross-linking, ligation and sequencing of hybrids (CLASH) (Helwak et al., 2013; Kudla et al., 2011) on E. coli. First, we demonstrate that the highly stringent purification steps make CLASH a robust method for direct mapping of Hfqmediated sRNA-target interactions in E. coli. This enabled us to significantly expand on the sRNA-target interaction repertoire found by RNase E CLASH (Waters et al., 2016) and RILseq (Melamed et al., 2016), and we show that Hfq CLASH can generate very reliable results. Using CLASH we identified many potentially novel 3'UTR-derived SRNAs, confirming that this class of sRNAs (Chao et al., 2012, 2017; Chao and Vogel, 2016; Miyakoshi et al., 2015a) is highly prevalent.

Next, we focussed our analyses on interactions that were specifically recovered during the transition phase where we identified a surprisingly large number of interactions, including sRNA-sRNA interactions. Our data suggests that during the transition stage, ArcZ represses CyaR levels, thereby indirectly controlling genes involving nutrient uptake during the transition phase. We also characterized a novel 3'UTR-derived sRNA, which we refer to as MdoR (maldependent OMP repressor). Unlike the majority of bacterial sRNAs, MdoR is transiently expressed during the transition phase. We demonstrate that MdoR is a degradation intermediate of the malG 3'UTR, the last transcript of the malEFG polycistron that encodes components of the maltose transport system. We show that MdoR directly downregulates several mRNAs encoding major porins and suppresses the envelope stress response controlled by $\sigma^{\mathrm{E}}$. We propose that MdoR is part of a regulatory network that, during the transition phase, promotes accumulation of high affinity maltose transporters in the outer membrane by repressing competing pathways.

\section{Results}

\section{Hfq CLASH in E. coli.}

To unravel the post-transcriptional networks that underlie the transition between exponential and stationary growth phases in E. coli, we performed CLASH (Helwak et al., 2013; Kudla et al., 2011) using Hfq as bait (Figure 1A). To generate high quality Hfq CLASH data made a number of improvements to the original protocol used for RNase E CLASH (Waters et al., 2016). 
A

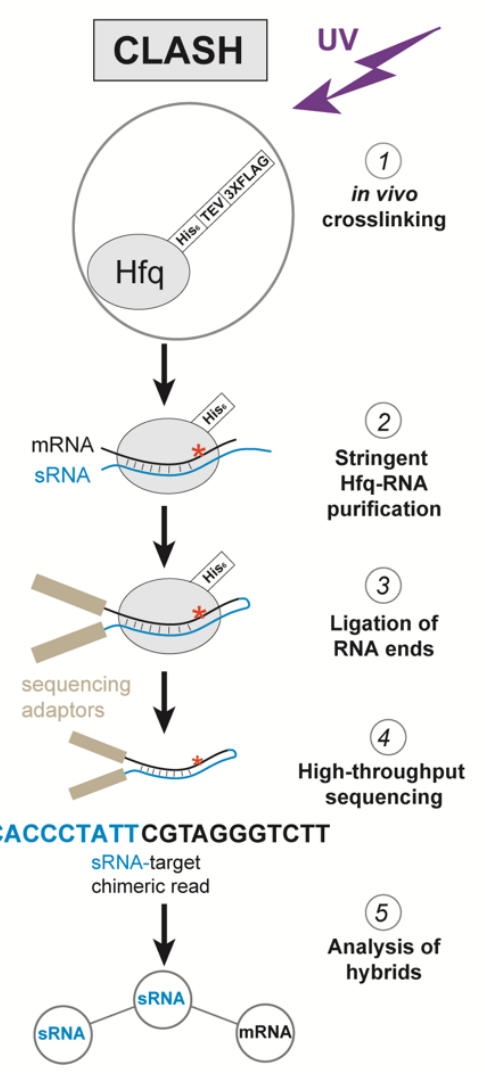

B

B Exponentiall $\stackrel{\text { Traition }}{\text { S }}$

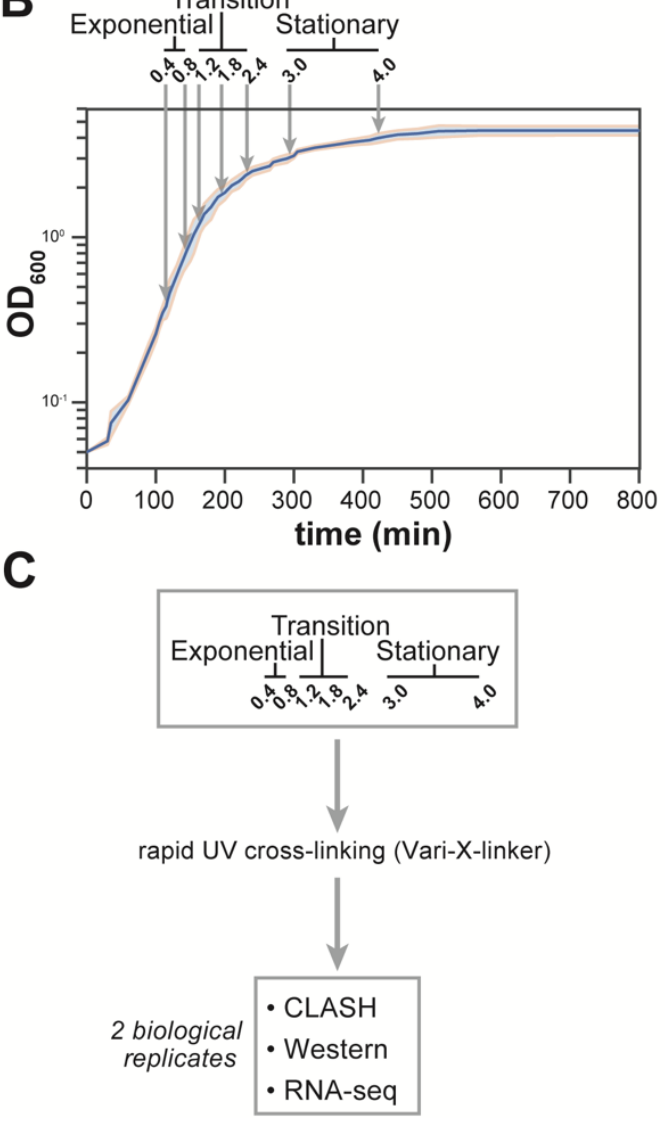

Figure 1. Hfq CLASH experiments at different growth phases in E. coli.

113 (A) Overview of the critical experimental steps for obtaining the Hfq CLASH data. E. coli cells expressing 114 an HTF (His6-TEV-3xFLAG)-tagged Hfq(Jai J. Tree et al., 2014) were grown in LB and an equal number 115 of cells were harvested at different optical densities $\left(\mathrm{OD}_{600}\right)$. Hfq binds to sRNA-target RNA duplexes, 116 and RNA ends that are in close proximity are ligated together. After removal of the protein, cDNA 117 libraries are prepared and sequenced. The single reads can be used to map Hfq-RNA interactions, 118 whereas the chimeric reads can be traced to sRNA-target interactions. (B) A growth curve of the 119 cultures used for the Hfq CLASH experiments, with $\mathrm{OD}_{600}$ at which cells were cross-linked indicated by 120 circles, and each growth stage is indicated above the plot. The results show the mean and standard 121 deviations of two biological replicates. Source data are provided as a Source Data file. (C) Cultures at 122 the same $\mathrm{OD}_{600}$ cross-linked and harvested by filtration were analysed by Hfq CLASH, RNA-seq and 123 Western blotting to detect Hfq.

Our Hfq CLASH protocol has several advantages over the related RIL-seq method 126 (see Materials and Methods and Discussion). As negative controls, replicate CLASH 127 experiments were performed on the untagged parental strain. When combined, the control 128 samples had $\sim 10$ times lower number of single-mapping reads and contained only 297 unique chimeric reads, compared to the over 70.000 chimeras identified in the tagged Hfq data. This demonstrates that the purification method produced very low background levels. 
131 Cell samples from seven different optical densities were subjected to Hfq CLASH.

132 Based on the growth curve analysis shown in Figure $1 \mathrm{~B}$, we categorized $\mathrm{OD}_{600}$ densities 0.4

133 and 0.8 as exponential growth phase, 1.2, 1.8, 2.4 as the transition phase from exponential to

134 stationary, and 3.0 and 4.0 as early stationary phase. To complement the CLASH data, RNA-

135 seq and Western blot analysis was performed on UV-irradiated cells to quantify steady state

136 RNA and Hfq protein levels, respectively (Figure 1C, Figure 1-figure supplement 1,

137 Supplementary Table 1). Western blot analyses revealed that Hfq levels gradually increased

138 during growth, however, when normalized to the levels of the chaperone GroEL, the increase

139 was modest (Figure 1-figure supplement 1A-B). To determine the cross-linking efficiency, Hfq-

140 RNA complexes immobilized on nickel beads were radiolabelled, resolved on NuPAGE gels

141 and detected by autoradiography. The data show that the recovery of Hfq and radioactive

142 signal was comparable at each optical density studied (Figure 1-figure supplement 1C).

143 Comparison of normalized read counts of replicate CLASH and RNA-seq experiments showed

144 that the results were highly reproducible (Figure 1-figure supplement 2).

145

Hfq binds to the transcriptome in a growth-stage dependent manner.

147 Meta-analyses of the $\mathrm{Hfq}$ CLASH sequencing data revealed that the distribution of $\mathrm{Hfq}$ 148 binding across mRNAs was very similar at each growth stage. We observed the expected Hfq 149 enrichment at the 5'UTRs and at the 3'UTRs at each growth stage (see Figure 1-figure 150 supplement 3A and 3B for examples). After identifying significantly enriched Hfq binding peaks

151 (FDR < = 0.05; see Methods for details) we used the genomic coordinates of these peaks to 152 search for Hfq binding motifs in mRNAs. The most enriched k-mer included poly-U stretches 153 (Figure 1-figure supplement 3C) that resemble the poly-U tracts characteristic to Rho154 independent terminators found at the end of many bacterial transcripts (Wilson and Hippel, 155 1995), and confirms the motif uncovered in CLIP-seq studies in Salmonella (Holmqvist et al., 156 2016). 
A
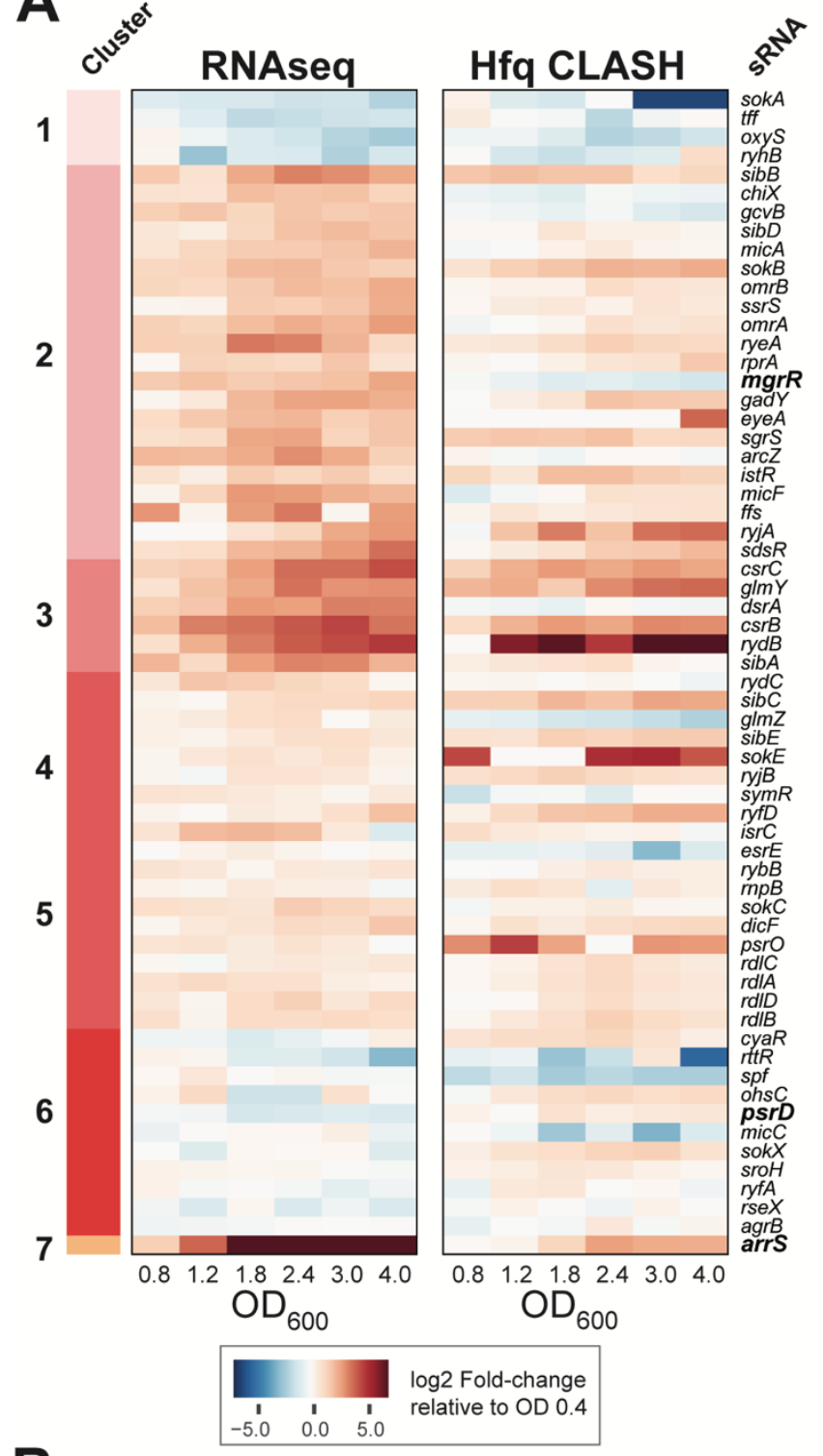

B

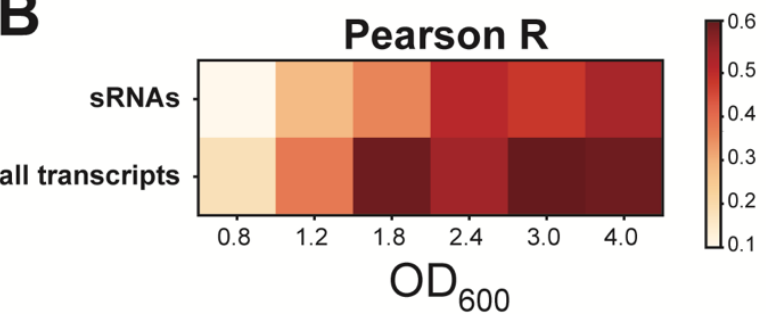

C

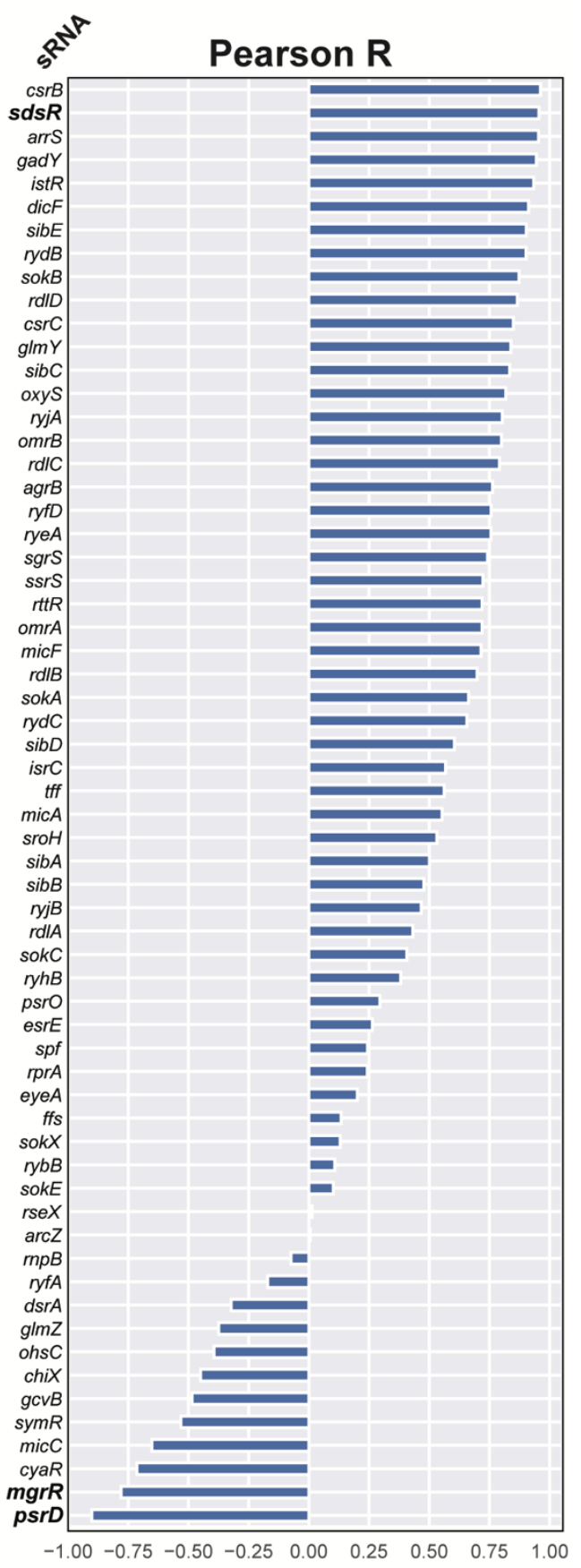

Figure 2. Hfq binding to sRNAs and sRNA steady state levels in $E$. coli do not always correlate highly.

161 (A) Heatmaps showing the changes in sRNA steady-states (Left) and Hfq cross-linking (Right) relative

162 to $\mathrm{OD}_{600}$ 0.4. The clustered RNA-seq data were generated by k-means clustering using the foldchanges of normalized counts ( $\log _{2}$ of transcripts per million (TPM)) relative to $\mathrm{OD}_{600} 0.4$. A blue shade indicates a reduction in levels compared to $\mathrm{OD}_{600} 0.4$, whereas a dark orange shade indicates an increase. The cluster assignment is indicated at the left, and the growth stage is indicated as $\mathrm{OD}_{600}$ units below each heatmap. Note that ssrS and ffs encode the cytoplasmic RNAs $6 S$, a regulator of RNA polymerase, and $4.5 \mathrm{~S}$, the signal recognition particle RNA. EyeA is an uncharacterized sRNA mapped by(Sætrom et al., 2005). (B) Global correlation of between Hfq binding and steady state RNA levels 
increases at higher cell densities. The heatmap shows the changes in Pearson R correlation between Hfq binding and RNA expression (normalised as in (a)), for sRNAs (top) and all transcripts (bottom). (C) Assessment of correlation between changes in expression and Hfq binding profiles for individual sRNAs. The y-axis shows the gene name of the sRNAs and the Pearson coefficient indicating the correlation between $\mathrm{Hfq}$ binding and steady state levels for each sRNA is shown on the x-axis.

Given the established role of Hfq in sRNA stabilization and mediating sRNA-target interactions, it was logical to assume that changes in Hfq binding would also be reflected in changes in sRNA steady-state levels. This would imply that the Hfq binding data would show a strong correlation with the RNA-seq data. To test this, we compared the Hfq cross-linking data to the RNA-seq data. K-means clustering of the normalized data revealed 7 different patterns of changes in normalised read counts in the Hfq cross-linking and total RNA-seq data (Figure 2A). Reminiscent of recent work performed in Salmonella (Chao et al., 2012), most sRNAs in E. coli appear to be preferentially expressed when the cells reach the transition and stationary phase (Figure 2A). However, much to our surprise, the Hfq cross-linking profile did not always follow the same trend (Figure 2A-C, Figure 2-figure supplement 1A). Globally, changes in sRNA expression did not correlate strongly with the Hfq-binding profile (and vice versa) (Fig 2B-C). The correlation between changes in sRNA expression levels versus changes in Hfq cross-linking was particularly poor at lower cell density $\left(\mathrm{OD}_{600} 0.8: r=0.10\right)$ but gradually improved as the cells approach stationary phase $\left(\mathrm{OD}_{600} 4.0 \mathrm{r}=0.54\right.$; Figure $2 \mathrm{~B}$ and Figure 2-figure supplement $1 \mathrm{~A}$ ). A very similar result was obtained when comparing all Hfq-bound RNAs, including mRNAs (Figure 2B and Figure 2-figure supplement 1B). Striking examples are MgrR and PsrD, which showed a strong anti-correlation between Hfq CLASH and RNA-seq counts (Figure $2 \mathrm{C}$ ) In the case of PsrD, Hfq binding showed a modest increase during the growth phase (Figure 2A; right heat map, cluster 6 ), whereas sRNA levels steadily decreased (Figure 2A; left heat map; cluster 2). PsrD/SraB has also been shown to bind ProQ (Smirnov et al., 2017), which may explain why its accumulation does not correlate with Hfq binding. On the other side of the spectrum, SdsR showed a very high positive Pearson correlation (Figure 2), suggesting that its accumulation heavily relies on $\mathrm{Hfq}$ binding.

We conclude that the dynamics of sRNA expression and binding to Hfq are not always highly correlated.

\section{Hfq CLASH robustly detects RNA-RNA interactions.}

To get a complete overview of the RNA-RNA interactions captured by $\mathrm{Hfq} \mathrm{CLASH}$, we merged the data from the two biological replicates of CLASH growth phase experiments (Supplementary Table 2.1). Overlapping paired-end reads were merged and unique chimeric 

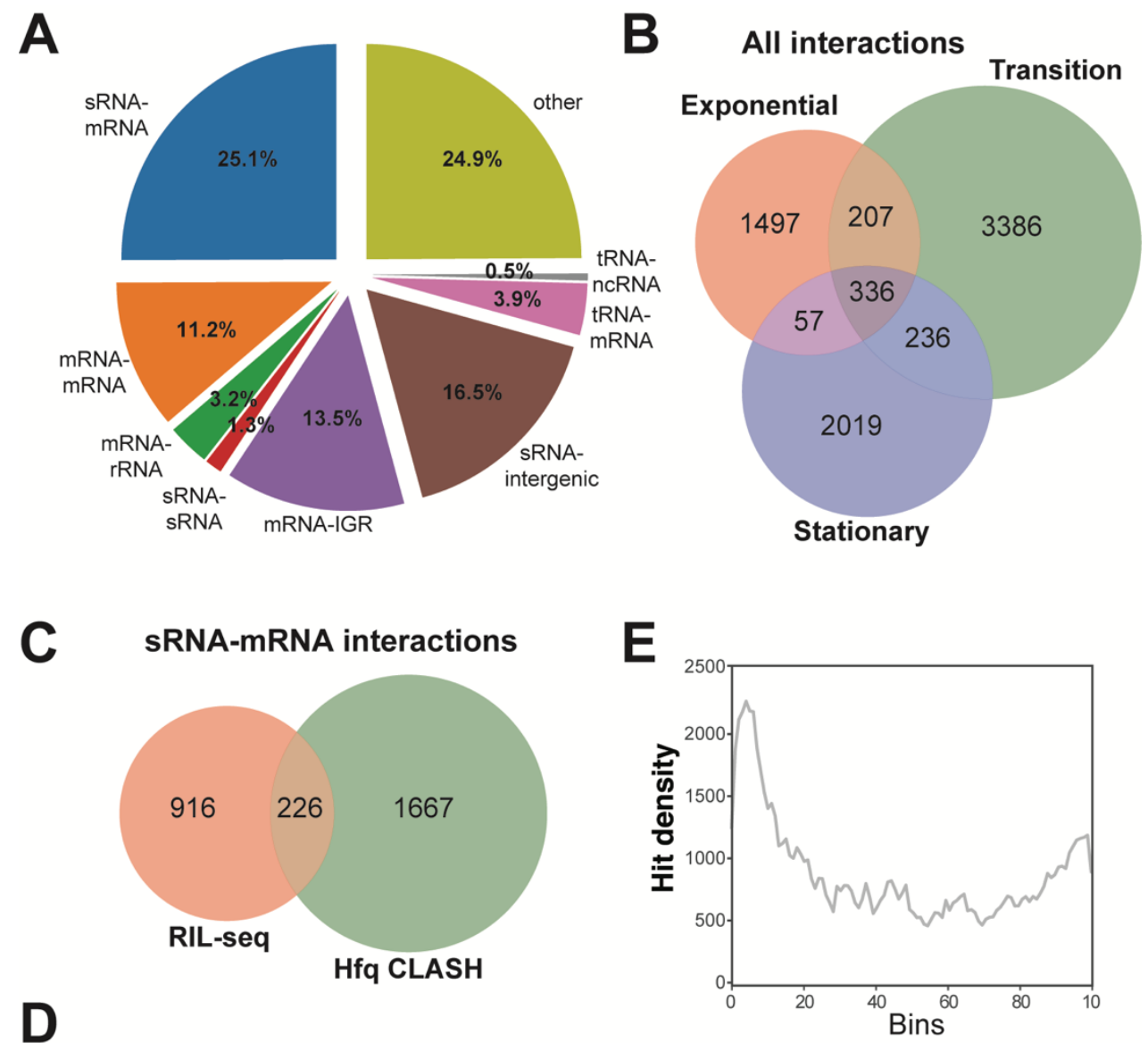

D

\section{SRNA-mRNA interactions}
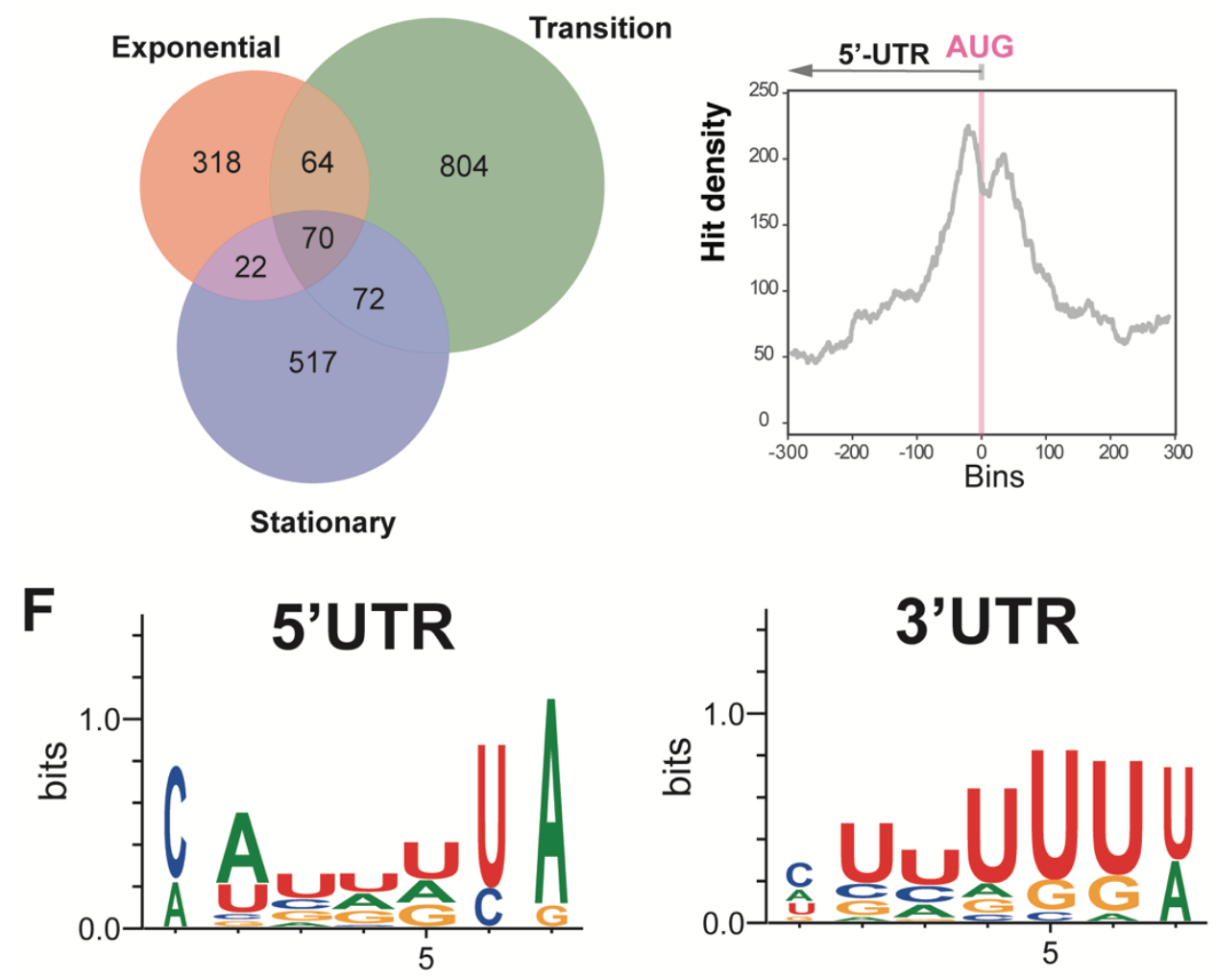

Figure 3. Hfq CLASH detects RNA-RNA interactions in E. coli. 
(A) Intermolecular transcript combinations found in interactions captured by Hfq CLASH. Combination count of all uniquely annotated hybrids on genomic features. ${ }^{*}$ tRNA-tRNA and rRNA-rRNA chimeras originating from different coding regions were removed. (B) Venn diagram showing the intersection between interactions from statistically filtered CLASH data from two biological replicates, recovered at three main growth stages: exponential ( $\mathrm{OD}_{600} 0.4$ and 0.8$)$, transition $\left(\mathrm{OD}_{600} 1.2,1.8,2.4\right)$ and early stationary (OD600 3.0 and 4.0). (C) Comparison of sRNA-mRNA interactions found in RIL-seq S-chimera data and Hfq CLASH data. (D) Same as in (B) but for sRNA-mRNA interactions. (E) (Top) Distribution of chimeras representing statistically filtered interactions, which uniquely map to mRNA genes. Overlapping fragments of the two individual parts of the chimeras were collapsed into single clusters followed by generation of distribution plots. Each gene was divided in 100 bins the number of clusters that map to each bin (hit density; y-axis) was calculated; (Bottom) For the distribution plot around the AUG, the gene length was normalized in 601 bins (x-axis) 5 '-end overlap $(-300)$ before the start of the coding sequence, and 300 bins downstream AUG (+300); the bins corresponding to the start codon are indicated with a pink line. (F) Enriched motifs in chimeras that uniquely overlap 5'UTRs and 3'UTRs; the logos were drawn using the top $20 \mathrm{~K}$-mers.

222

reads were identified using the hyb pipeline (Travis et al., 2013). To select RNA-RNA interactions for further analysis, we applied a probabilistic analysis pipeline previously used for the analysis of RNA-RNA interactions in human cells (Sharma et al., 2016) and adapted it for the analyses of RNase E CLASH data (Waters et al., 2016). This pipeline tests the likelihood that observed interactions could have formed spuriously. Strikingly, $87 \%$ of the chimeric reads had a Benjamini-Hochberg adjusted p-value of 0.05 or less, indicating that it is highly unlikely that these interactions were generated by random ligation of RNA molecules. These analyses demonstrate the robustness of Hfq CLASH protocol. A complete overview of statistically significantly enriched chimeras is provided in Supplementary Table 2.2.

The distribution of combinations of transcript classes found in the statistically filtered chimeric reads indicates the sRNA-mRNA interactions as the most frequent recovered Hfqmediated interaction type ( $25 \%$; Figure $3 A$ ). We suspect that this number might be higher, as about $16 \%$ of the chimeras contained SRNA and fragments that mapped to intergenic regions (Figure $3 \mathrm{~A}$ ). Manual inspection of several of these indicated that some of the intergenic sequences were located near genes for which the UTRs were unannotated or short. The vast majority of these interactions were growth stage-specific (Figure 3B). Hfq CLASH identified almost 2000 sRNA-mRNA interactions (Figure 3C; Supplementary Table 2). Around 20\% of

240 the interactions found with RIL-seq and $\sim 21 \%$ (27 out of 126) of the experimentally verified interactions present in sRNATarbase3 (Supplementary Table 2.7) were recovered. These results suggest that while the CLASH data contained known and many novel interactions, the analyses clearly were not exhaustive. 
Meta-analysis revealed that the majority of interactions were identified in the transition phase (Figure 3D) and that the mRNA fragments found in chimeric reads were strongly enriched in 5'UTRs peaking near the translational start codon (Figure 3E). The latter is consistent with the canonical mode of translational inhibition by sRNAs (Bouvier et al., 2008) and demonstrates the robustness of the data. Enrichment was also found in 3'UTRs of mRNAs (Figure 3F). Motif analyses revealed a distinct sequence preference in 5'UTR and 3'UTR fragments are more consistent with Hfq binding to Shine Dalgarno-like (ARN) $)_{n}$ sequences (Jai J. Tree et al., 2014) and U-tracts, whereas the 3'UTR-containing chimera consensus motif corresponds to poly-U transcription termination sites (Figure 3F and Supplementary Table 3).

\section{Hfq CLASH predicts sRNA-sRNA interactions as a widespread layer of post-} transcriptional regulation.

We also uncovered a surprisingly large number of SRNA-sRNA interactions (Supplementary Table 2.4), many of which were uniquely found in our Hfq CLASH data (Figure 4A). Many interactions were growth-stage specific and the sRNA-sRNA networks show extensive rewiring across the exponential, transition and stationary phases (Figure 4-figure supplement 1). The sRNA-sRNA network is dominated by several abundant sRNAs that appear to act as hubs that have many interacting partners: ChiX, Spot42 (spf), ArcZ and GcvB. In many cases the experimentally-validated sRNA seed sequences were found in the chimeric reads, for both established and novel interactions. For example, the vast majority of ArcZ sRNA-sRNA chimeras contained the known and well conserved seed sequence (Figure 4B, Figure 4-figure supplement 2).

The sRNA-sRNA chimeras containing CyaR fragments were of particular interest, as the sRNA is primarily expressed during the transition from late exponential to stationary phase (De Lay and Gottesman, 2009). In the case of CyaR, the known seed sequence (De Lay and Gottesman, 2009; Papenfort et al., 2008) as well as a conserved $\sim 25 \mathrm{nt}$ fragment in the 5' region was found in chimeras (Figure 4-C; Figure 4-figure supplement 2). Similar seed sequences were identified in CLASH experiments using RNase $\mathrm{E}$ as a bait (Waters et al., 2016), suggesting that this region represents a bona fide interaction site. Notably, we identified ArcZ-CyaR chimeras containing the seed sequence from both sRNAs (Figure 4-figure supplement 2) and these were detected specifically in the transition phase (Figure 4B-C), suggesting that these sRNAs could influence each other's activity. To validate these findings, we used an E. coli plasmid-based assay that is routinely used to monitor sRNA-sRNA interactions and expression of their target mRNAs (Melamed et al., 2016; Miyakoshi et al., 2015b; Jai J. Tree et al., 2014). An advantage of this system is that each sRNA would be uncoupled from the chromosomally encoded regulatory networks (that were thought to act 
largely in a 1:1 stoichiometry) and to allow the specific effects of the sRNA-target RNA to be assessed (Miyakoshi et al., 2015b). Importantly, these sRNAs were induced during early exponential growth phase when the endogenous (processed) ArcZ and CyaR sRNAs are detectable at only very low levels (Figure 4-figure supplement 3B, lanes 1, 2, 5, 7). The qPCR data were subsequently normalized to the results obtained with a control scrambled SRNA to calculate fold changes in expression levels. Since it is difficult to predict directly from the CLASH data which sRNA in each pair acts as the decoy/sponge, we tested both directions. ArcZ overexpression not only decreased the expression of its mRNA targets (tpx, sdaC) by more than $50 \%$ but also that of CyaR (Figure 5D, panel I). Concomitantly, we observed a substantial increase in CyaR targets nadE and yqaE (Figure 4D, panel I). CyaR overexpression reduced the level of a direct mRNA target (nadE) by $\sim 40 \%$ but it did not significantly alter the level of ArcZ or ArcZ mRNA targets (tpx and sdaC; Figure 4D, panel II). Notably, in this two-plasmid assay CyaR was not expressed at levels higher than ArcZ (Figure 4-figure supplement 3A, panel II). Therefore, it is possible that under the tested conditions the CyaR overexpression was not sufficient to see an effect on ArcZ. We find this unlikely as overexpression of CyaR also did not significantly affect endogenous ArcZ levels, which was 80-fold less abundant than CyaR in this experiment (Figure 4-figure supplement 3A, panel III). The qPCR results were also confirmed by Northern blot analyses (Figure 4-figure supplement 3B, lanes 1-8), which also demonstrate that ArcZ processing was not affected upon CyaR overexpression. These results suggest that the regulation is unidirectional, reminiscent of what has been described Qrr3 in Vibrio harveyi (Feng et al., 2015). We conclude that ArcZ acts as a CyaR anti-sRNA and can trigger its degradation.

To provide additional support for direct interactions between these sRNAs, we generated mutations in the seed sequences of the sRNAs analysed here (Figure 4C). We found that two $G$ to $C$ nucleotide substitutions in ArcZ was sufficient to disrupt ArcZ downregulation of CyaR (Figure 4C-D; ArcZ 70-71 + CyaR). This regulation, however, was almost fully restored when complementary mutations were introduced in the CyaR region (Figure 5C-D; ArcZ 70-71 + CyaR 38-39). These data also demonstrate that it is very unlikely that the observed changes in CyaR levels were be the result of Hfq redistribution due to overexpression of ArcZ over-expression (Moon and Gottesman, 2011; Papenfort et al., 2009), as the ArcZ seed mutant stably accumulated (and therefore effectively binds $\mathrm{Hfq}$ ), but did not affect CyaR levels. Unexpectedly, the wild-type ArcZ was also able to effectively suppress the CyaR seed mutant (Figure 5D; ArcZ + CyaR 38-39), possibly because ArcZ can still form stable base-pairing interactions with the CyaR mutant.

These results, together with the CLASH data, strongly support the notion that ArcZ and CyaR base-pair in vivo, resulting in degradation of CyaR but not vice versa. 
A

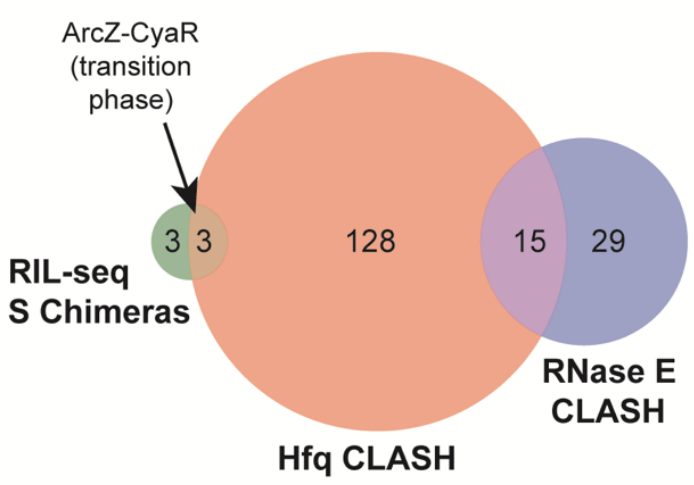

B

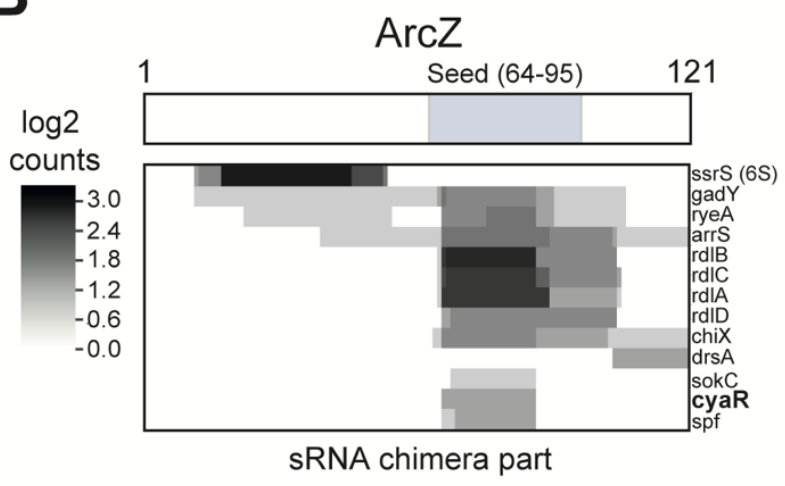

CyaR

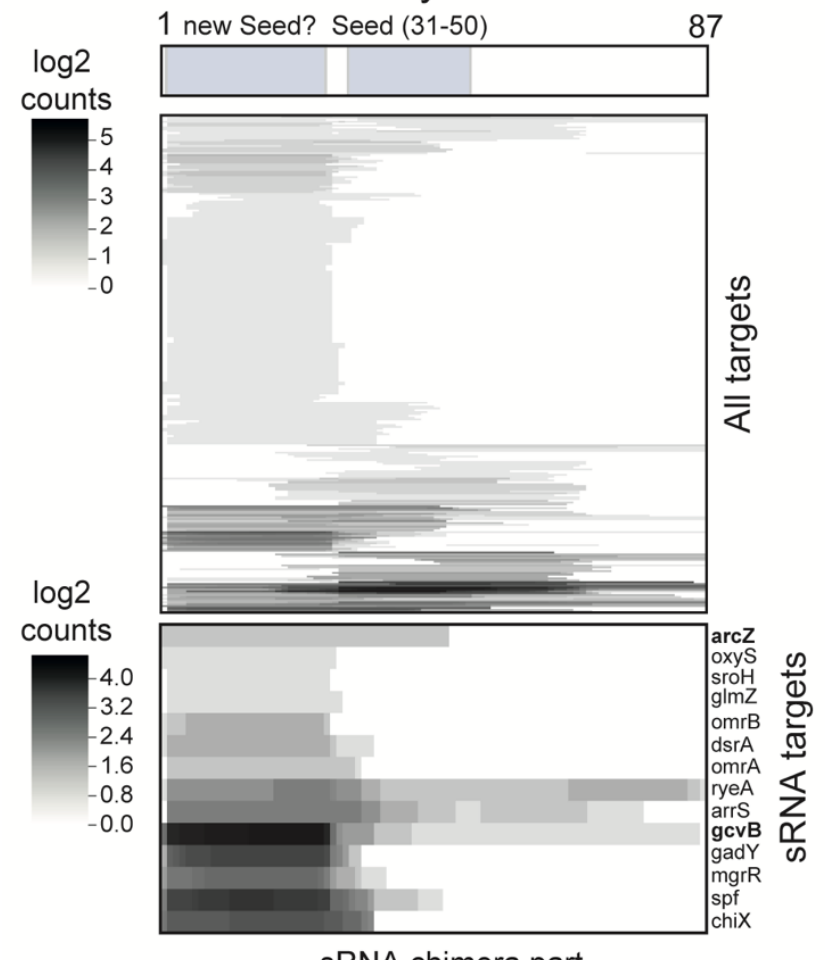

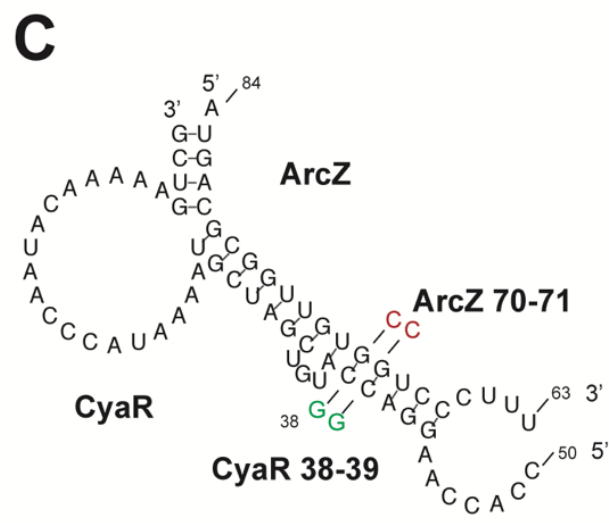

D
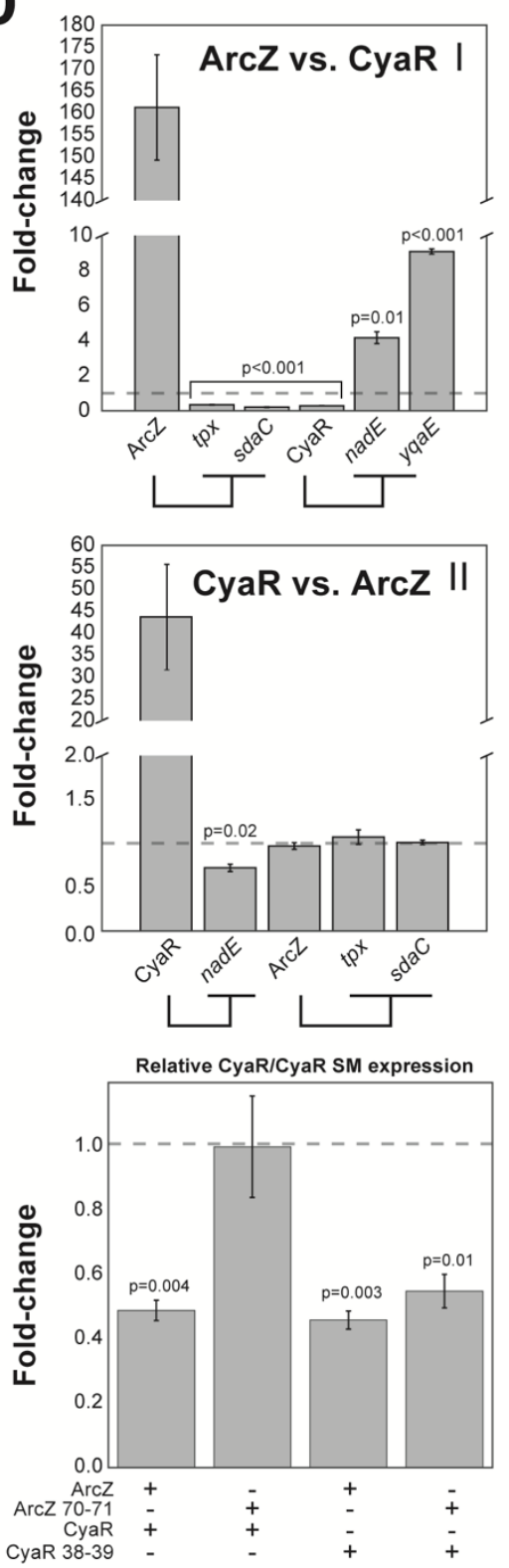

Figure 4. sRNA-RNA interactions identified by CLASH are growth-stage specific.

(A) Hfq CLASH uncovers sRNA-sRNA networks: comparison between statistically filtered sRNA-sRNA interactions in the Hfq CLASH data, RIL-seq S-chimeras (Melamed et al., 2016) (log and stationary) 
and RNase E CLASH (Waters et al., 2016). Only core genome sRNAs were considered. Red coloured sRNA-sRNA interactions have been characterized in more detail. (B) Heatmaps showing the read density ( $\log _{2}$ (chimera count)) of chimeric fragments mapping to ArcZ and CyaR. (Top) ArcZ regions involved in sRNA-sRNA interactions. The location of the known ArcZ seed sequence is indicated above. (Bottom) CyaR heatmaps that show all CyaR chimeras and CyaR-sRNA chimeras, respectively. The location of the known CyaR seed sequence, as well as a new seed, is indicated above. (C) Base-pairing interactions predicted from the ArcZ-CyaR chimeras using RNAcofold. The nucleotide substitutions for experimental validation of direct base-pairing are shown as red or green residues. (D) SRNA-sRNA interactions coordinate nutritional stress responses. ArcZ, CyaR were overexpressed and the levels of their targets were monitored by RT-qPCR. The tpx and sdaC mRNAs are ArcZ mRNA targets. The nadE and yqaE mRNAs are CyaR targets. The dppA mRNA is a GcvB target. Experiments were performed in biological and technical triplicates; Error bars indicate the standard error of the mean (SEM) of the three biological replicates. The dashed horizontal line indicates the level of the overexpressed scrambled RNA. (E) ArcZ and CyaR directly interact. The sRNAs and mutants as in (C) were ectopically co-expressed in E. coli and CyaR and CyaR 38-39 levels were quantified by RT-qPCR.

\section{Hfq CLASH identifies novel sRNAs in untranslated regions}

Two lines of evidence from our data indicate that many more mRNAs may be harbouring sRNAs in their UTRs or be involved in base-pairing among themselves. First, around $11 \%$ of the intermolecular chimeras mapped to mRNA-mRNA interactions (Figure $3 \mathrm{~A}$ ). Secondly, we observed extensive binding of Hfq in 3'UTRs near transcriptional terminators (Figure 1-figure supplement 3A, C), indicating that like in Salmonella, E. coli 3'UTRs may harbour many functional sRNAs (Chao et al., 2017). We identified 122 3'UTR-containing mRNA fragments that were involved in 550 interactions. Sixty-five of these interactions were also identified in the RIL-seq S-chimeras data (Melamed et al., 2016). Eighteen of the 3'UTRs were found as part of chimeras in the RIL-seq data, while 10 appeared stabilised upon transient inactivation of RNase E performed in Salmonella (TIER-seq data (Chao et al., 2017)); Figure 6A, Supplementary Tables 2.5 and 2.6). Out of the 550 3'UTR-mRNA chimeric reads, 79 were 3'UTRs fused to 5'UTRs of mRNAs, suggesting that these may represent 3'UTRderived sRNAs that base-pair with 5'UTRs of mRNAs, a region frequently targeted by sRNAs (Supplementary Table 2.6). Strikingly, 223 interactions contained 3'UTR fragment of $c p x P, 51$ of which were also found in the RIL-Seq data (Supplementary Table 2.6). In Salmonella cpxP harbours the CpxQ sRNA (Chao and Vogel, 2016). Our analyses greatly increased the number of potential $C p x Q$ mRNA targets and show that the vast majority of $C p x Q$ interactions take place during the transition and stationary phases (Supplementary Table 2.6). 
bioRxiv preprint doi: https://doi.org/10.1101/481986; this version posted August 7, 2019. The copyright holder for this preprint (which was

not certified by peer review) is the author/funder, who has granted bioRxiv a license to display the preprint in perpetuity. It is made available under aCC-BY-NC-ND 4.0 International license.

A malG (MdoR), malM, sucD (SdhX), cutC (MicL)

\section{E. coli RIL-seq 3'UTR gltI (SroC)}

12

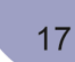

B
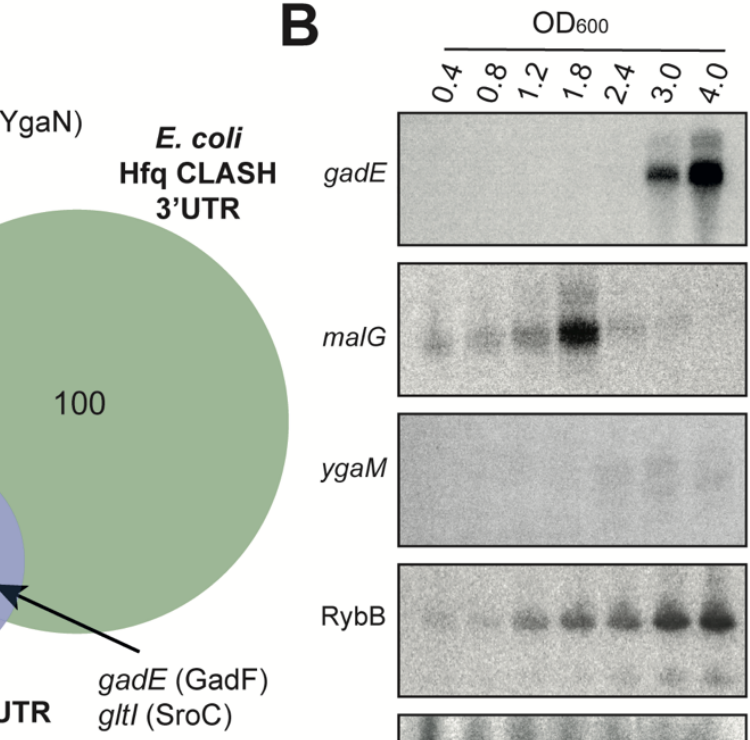

RybB

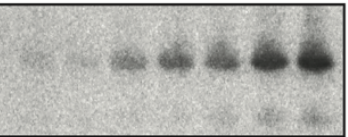

C
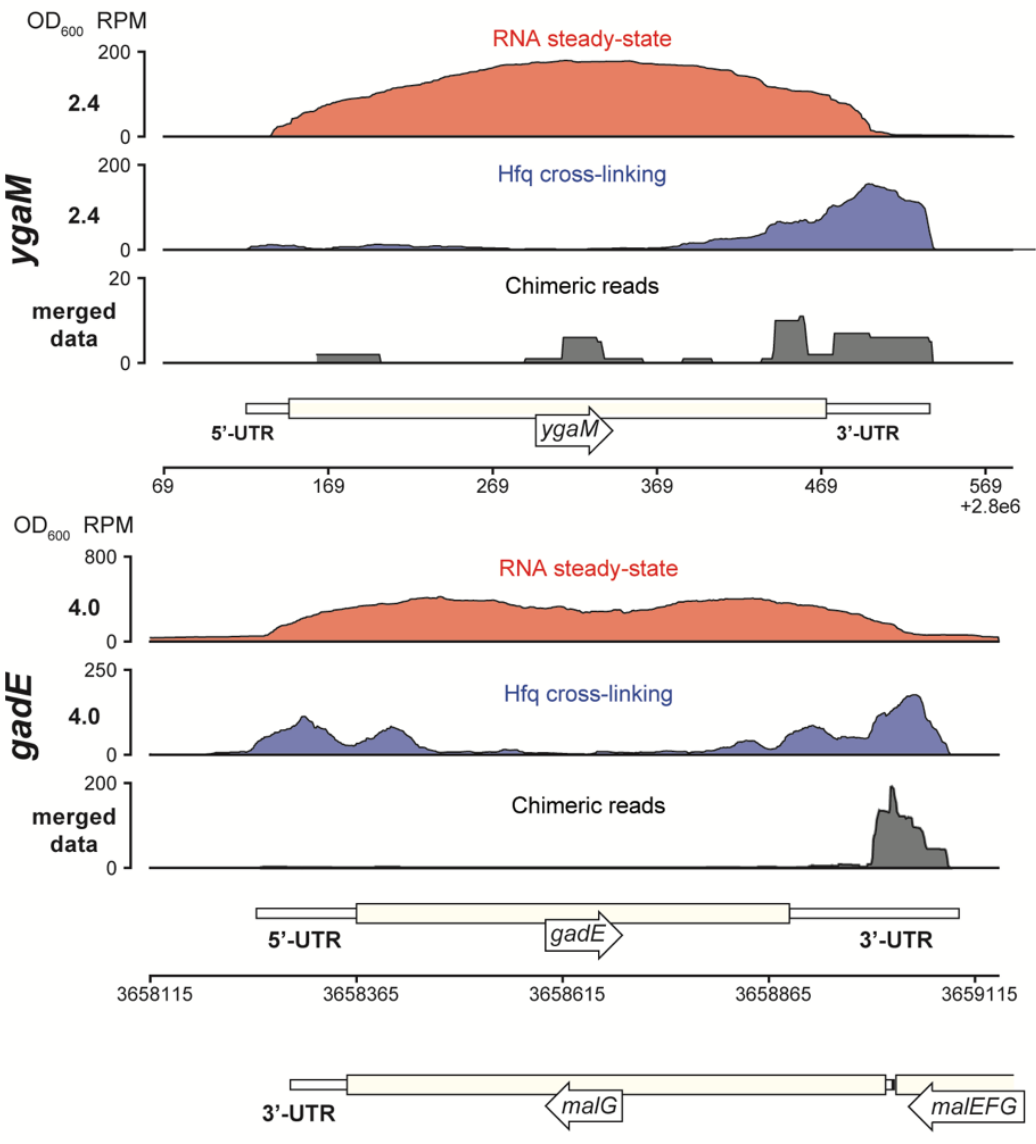

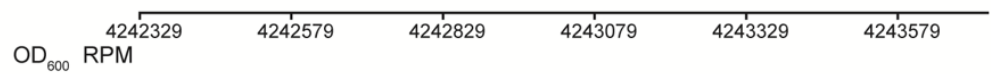
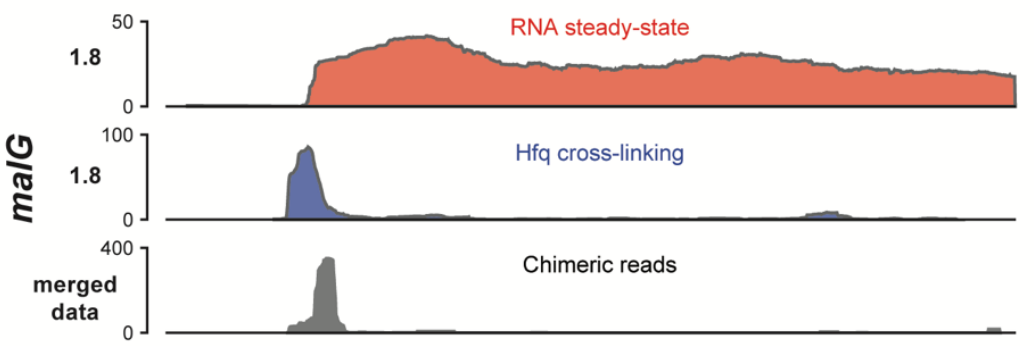
Figure 5. Hfq CLASH uncovers novel 3'UTR-derived sRNAs.

361 (A) Genes of which the 3'UTRs were found fused to mRNAs, were selected from the statistically filtered

362 CLASH data and RIL-seq S-chimera data. The RIL-seq RNA-RNA interaction set (Melamed et al., 2016)

363 S-chimeras for Log and Stationary phases of growth was filtered for the 3'UTR/EST3UTR annotations

364 on either orientation of the mRNA-mRNA pairs. Both were intersected with the set of mRNAs that were

365 predicted by TIER-seq studies (Chao et al., 2017) to harbour sRNAs that get released from 3'UTRs by

366 RNase E processing. Known (CpxQ, SdhX, MicL, GadF and SroC) and novel 3'UTR derived sRNAs

367 (MdoR, figL 3'UTR and YgaN) are indicated. (B) MdoR is transiently expressed during the transition

368 from exponential to stationary phase. RybB was probed as a sRNA positive control and 5S rRNA as

369 the loading control. See Figure 6-figure supplement 1 for full-size blots. Source data are provided as a

370 Source Data file. (C) Genome-browser snapshots of several regions containing candidate sRNAs for

371 optical densities at which the RNA steady-state was maximal for each candidate; the candidate names

372 and $\mathrm{OD}_{600}$ are indicated at the left side of the $y$-axes; the y-axis shows the normalized reads (RPM:

373 reads per million); red: RPM of RNA steady-states from an RNA-seq experiment, blue: Hfq cross-linking

374 from a CLASH experiment; black: unique chimeric reads found in this region.

375

We identified six mRNA 3'UTRs that were uncovered in all three (Hfq CLASH, RIL-seq and TIER-seq) datasets (Figure $5 \mathrm{~A}$ ), suggesting these likely contain sRNAs released from 3'UTRs by RNase E processing. Northern blot analyses confirmed the presence of sRNAs in malG and gadE 3'UTRs (Figure 5B, Figure 5-figure supplement 1A). The latter was also recently experimentally confirmed in the RIL-seq data and was annotated as GadF (Melamed et al., 2016). Furthermore, significant Hfq cross-linking could be detected in the 3'UTRs of these transcripts (Figure 5C). In addition, we could show that the 3'UTR of ygaM, which was found in chimeric reads in our data, also likely harbours a $\sim 100$ nt sRNA (hereafter referred to as YgaN; Figure 5-figure supplement $1 \mathrm{~A}$ ) and robust Hfq cross-linking could be detected in this region (Figure $5 \mathrm{~B}-\mathrm{C}$ ).

To substantiate these results, we analysed RNA-seq data from a study that used Terminator 5'-Phosphate Dependent Exonuclease (TEX) to map transcription start sites (TSS) of coding and non-coding RNAs in E. coli (Thomason et al., 2015). TEX degrades processed transcripts that have 5' monophosphates, but not primary transcripts with 5' triphosphates. Therefore, these data enabled us to determine whether (a) a TSS was detected in the 3'UTR and whether these were generated by RNase-dependent processing (TEX sensitive) or originated from an independent promoter (TEX insensitive). For 47 of the 122 predicted 3'UTR-derived SRNAs TEX data provided strong evidence for the presence of sRNAs (Figure 5-figure supplement 1B-C, Supplementary Table 2.5 and see Data and Code availability). The TEX data indicate that ygaM has (at least) two promoters, one of which is located near the 3' end of the gene that we predict is the TSS for YgaN (Figure 5-figure supplement 1B). Furthermore, we speculate that $\mathrm{YgaN}$ is processed by RNases. This is based 
on the observation that multiple YgaN species were detected in the Northern blot analyses

399 (Figure 5-figure supplement 1A) and the TEX data indicate that shorter YgaN RNAs are 400 sensitive to TEX treatment (Figure 5-figure supplement 1B).

401 The majority of the sRNAs we analysed are more abundant at higher cell densities 402 (including GadF, YgaN and RybB; see Figure 5B, 2A). In sharp contrast, 3' UTR malG sRNA 403 was expressed very transiently and peaked at an $\mathrm{OD}_{600}$ of 1.8 (Figure $5 \mathrm{~B}$ ). We envisage that 404 the particularly transient expression of this sRNA may be correlated with a role in the adaptive 405 responses triggered during transition from exponential to stationary phases of growth. We named it MdoR (mal-dependent OMP repressor) and characterized it in detail.

The steady state levels of malG and malEFG transcripts recapitulate the same 408 expression profile: both peak at $\mathrm{OD}_{600}$ of 1.8 and drop to very low levels at $\mathrm{OD}_{600} 2.4$ (Figure 409 6A). Additionally, we identified shorter malG 3'UTR-containing fragments of intermediate 410 length between malG and MdoR that could be degradation intermediates (Figure 6B). The 411 detection of these intermediate species suggests that the malEFG primary transcript is 412 undergoing serial ribonucleolytic cleavage steps.

413 MdoR is a 104 nt sRNA that contains part of the malG coding sequence, including the 414 stop codon and the Rho-independent terminator (Figure 5-figure supplement $1 A-B$ ). Two lines 415 of evidence suggest that MdoR is generated via endonucleolytic cleavage: RNase E cleavage 416 was detected in the 3'UTR of malG in the Salmonella TIER-seq data (Chao et al., 2017). 417 Secondly, the TEX RNA-seq data supported the existence of a short RNA in the same region 418 that has a 5' monophosphate (Figure 5-figure supplement 1B-C). We verified the MdoR TEX 419 data by Northern blot analyses (Figure 6C). Consistent with the TEX RNA-seq data, MdoR 420 could not be detected in our RNA samples treated with TEX (Figure 6C, lanes 2 and 4), 421 confirming it bears a 5' monophosphate. Like MdoR, the positive control CpxQ (Chao and 422 Vogel, 2016) was degraded in the presence of TEX (Figure 6C). In contrast, RybB, an sRNA 423 with a 5' triphosphate transcribed from an independent promoter (Johansen et al., 2006; 424 Papenfort et al., 2006) was a poor substrate for the exonuclease (Figure 6C). 


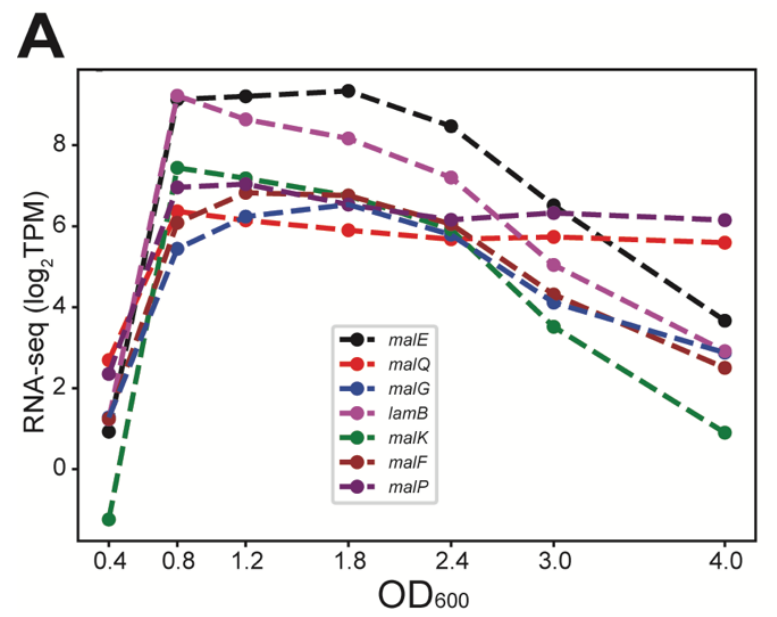

C
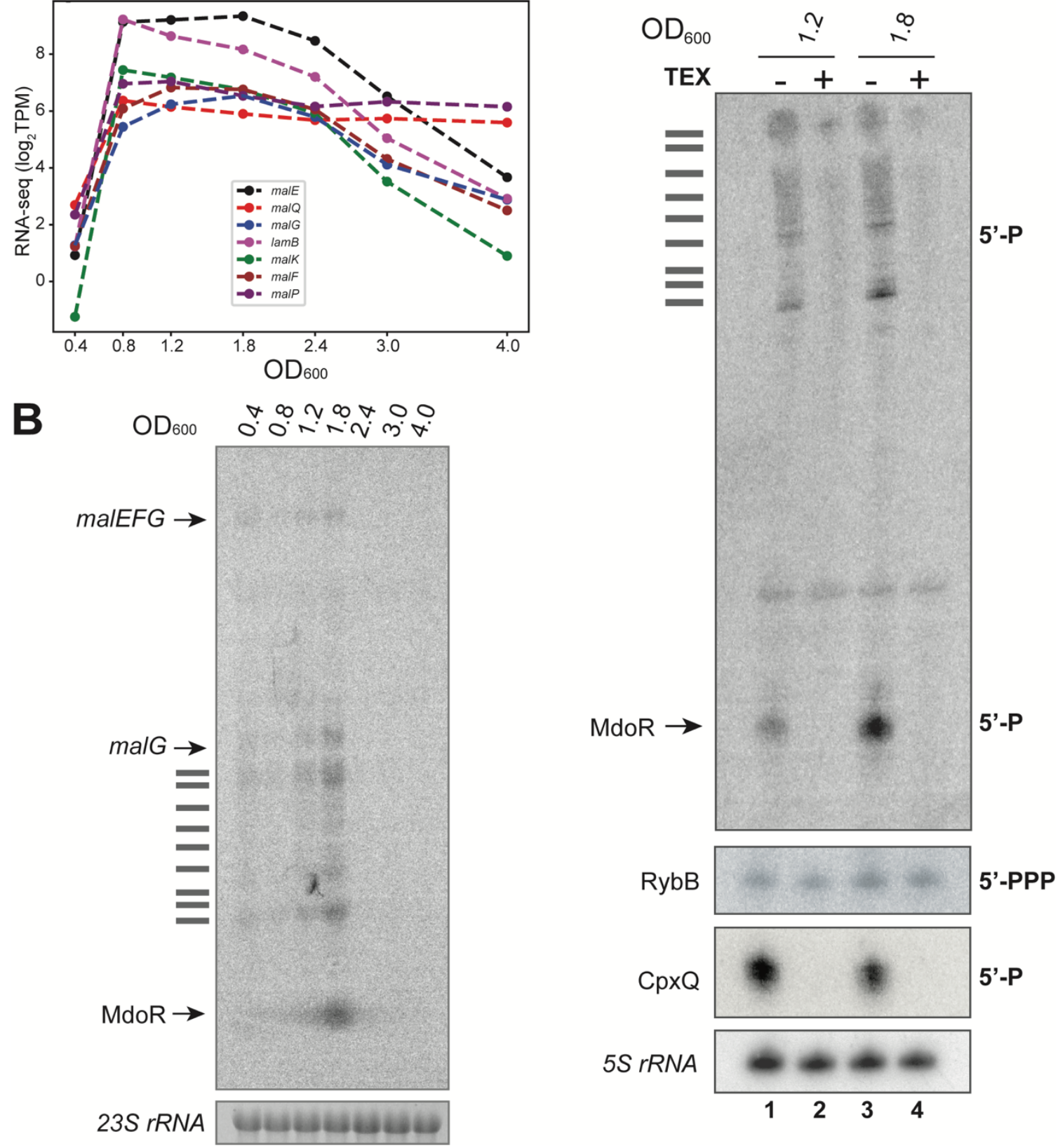

Figure 6. MdoR is a degradation product that emerges at the transition between exponential and stationary phase of growth.

429 (A) The mal regulon gene expression peaks at the transition between exponential and stationary phases of growth: the plot shows averages of $\log _{2}$ (TPM) normalized RNA steady-state levels ( $y$-axis); the $x$-axis indicates the cell densities $\left(\mathrm{OD}_{600}\right)$ at which samples were taken. (B) Northern blot using total RNA from $E$. coli harvested at different cell densities $\left(\mathrm{OD}_{600}\right)$ probed with an oligo antisense to malG 3'UTR; 23 S rRNA was used as the loading control; the identity of the bands is indicated at the left of the panel; horizontal bars indicate malEFG degradation intermediates. (C) MdoR is a degradation product: Northern blot using total RNA from cells at indicated OD 600 with (+; lanes 2 and 4) or without (; lanes 1 and 3) 5'-Phosphate-Dependent Exonuclease (TEX) treatment. The sRNAs RybB (5'ppp) and $\mathrm{CpxQ}\left(5^{\prime} p\right)$ were used as negative and positive controls, respectively. The 5S rRNA is a loading control. 
The text on the right of the blot indicates the phosphorylation state of the 5'-termini for each sRNA.

TEX treatment of the total RNA also reduced the levels of the longer intermediate species as well as the full-length $m a / G$. These data support a mechanism by which the fulllength polycistronic RNA undergoes decay that is initiated at a site in the upstream malEFG region. The distal gene malE is clipped off by the degradosome and selectively stabilized, allowing it to be expressed at higher levels than other members of the operon (Newbury et al., 1987). The malG 3'UTR, however, would be less susceptible to degradation as it is stabilized by Hfq binding.

MdoR directly regulates the expression of major outer membrane porins and represses

The MdoR chimeras frequently contained 5'UTR fragments of two mRNAs encoding major porins, ompC and ompA (Figure $7 \mathrm{~A}-\mathrm{C}$ ), which were also significantly enriched in the RIL-seq data (Figure 8B). The Hfq CLASH data, however, also contained MdoR fragments fused to a cis-encoded sRNA, OhsC (Figure 7B-C), suggesting that either MdoR controls its expression by sponging/degradation, or vice versa). The most abundant and favourable interactions of MdoR with mRNAs (ompC and ompA) appear to be utilizing roughly the same region of the malG 3'UTR for base-pairing (Figure 7C), suggesting that the corresponding site on the predicted sRNA may be a main, functional seed. The predicted interaction between MdoR and ompC is unusually long and consists of two stems interrupted by a bulge, suggesting that these two RNAs form a stable complex. Conservation analyses and in silico target predictions (CopraRNA (Wright et al., 2014, 2013)) indicate that the seed sequence predicted by CLASH is relatively well-conserved (Figure 7-figure supplement $1 \mathrm{~A}$ ), and could be utilized for the regulation of multiple targets (Figure 7-figure supplement $1 \mathrm{~B}$ ).

To verify the MdoR CLASH data we pulse-overexpressed the sRNA from a plasmidborne arabinose inducible promoter followed by RNA sequencing (Figure 7D-E). To minimize secondary changes in gene expression, cells were harvested after only 15 minutes of MdoR induction. Note that induction was performed at $\mathrm{OD}_{600}=0.4$, when endogenous levels of $\mathrm{MdoR}$ are very low. As a control we used cells harbouring an empty vector. Differential gene expression analysis (DESeq2 (Love et al., 2014)), identified $\sim 20$ transcripts that were significantly enriched in the control data compared to the MdoR overexpression data (Figure 7E-F; Supplementary Table 4). Thus, these transcripts are likely downregulated by MdoR in vivo. This set of transcripts included the sigma factor $r p o E\left(\sigma^{\mathrm{E}}\right)$, which plays an important role in controlling gene expression during stress responses, including envelope stress (Alba and

473 Gross, 2004; Bossi et al., 2008; De Las Peñas et al., 1997; Rhodius et al., 2006). The 
475 that $\sigma^{\mathrm{E}}$ and RseA are encoded by the same operon.

476

A

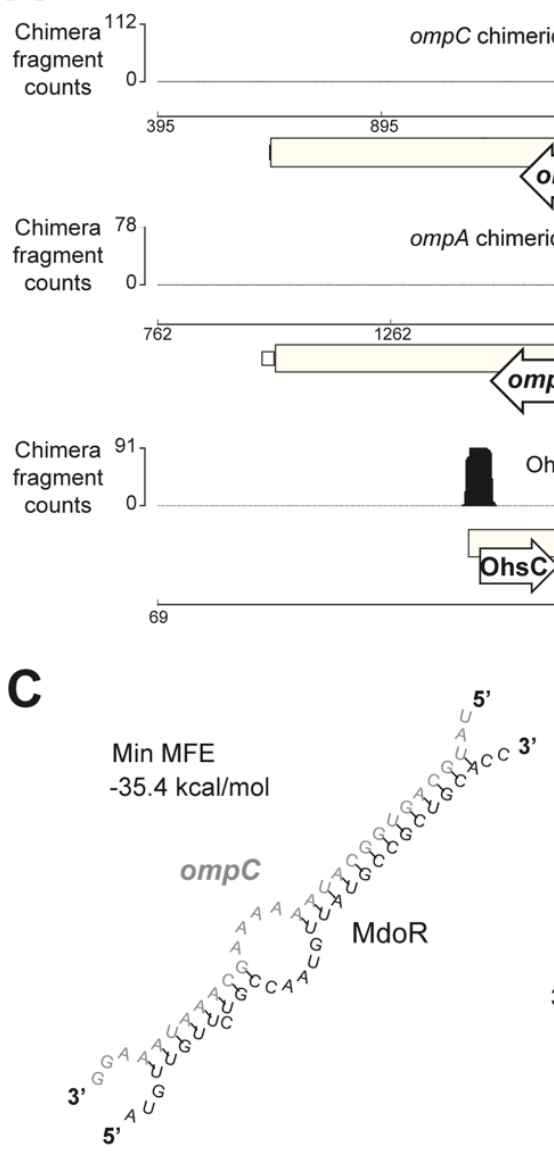

D

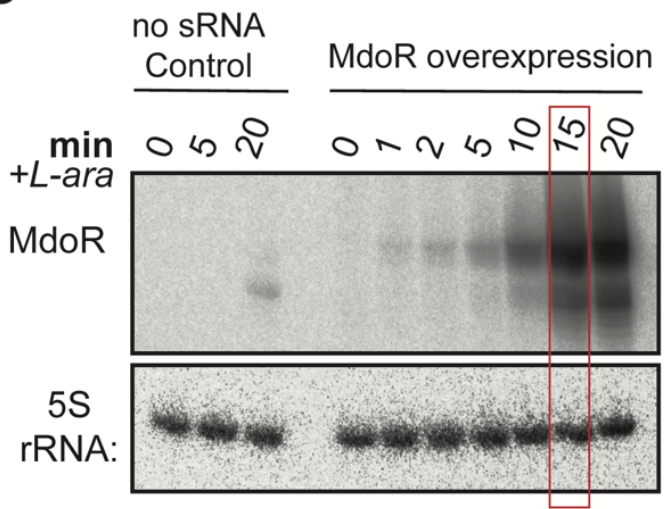

Min MFE

$-36.4 \mathrm{kcal} / \mathrm{mol}$

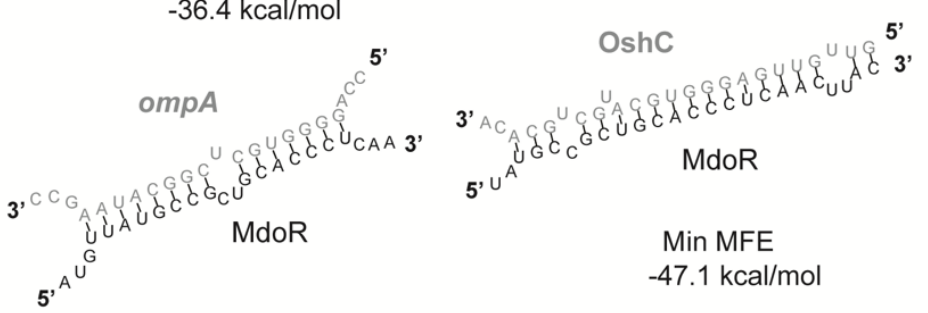

F

E

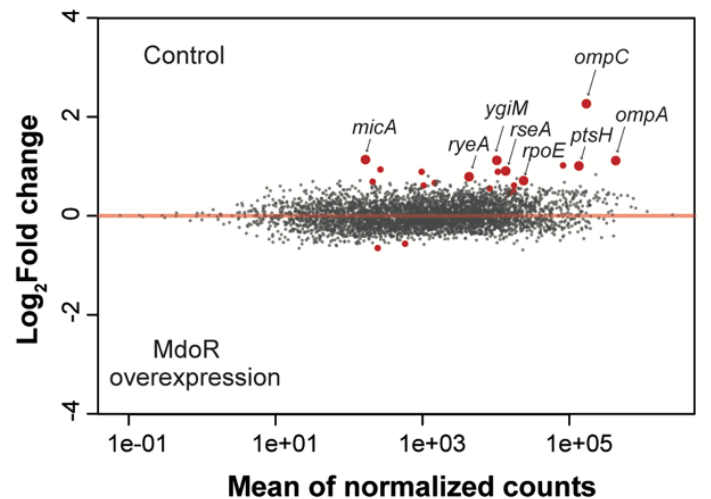

bacA-MdoR ompA-MdoR ompC-MdoR

ptsl-MdoR OhsC-MdoR ptsH-MdoR mutL-malG

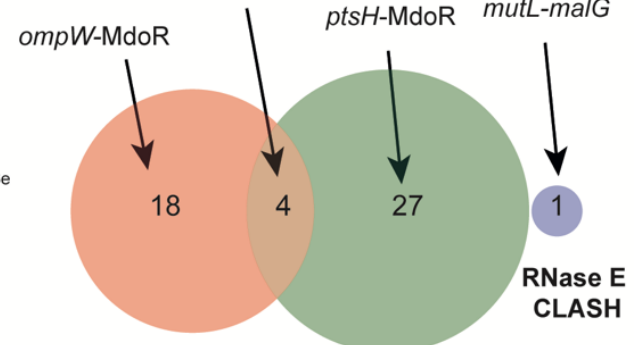

RIL-seq Hfq CLASH CLASH

B 
480 (A) The three genome browser tracks show the distribution of target mRNA and sRNA fragments

481 (ompC, ompA and OhsC) that were fused to MdoR fragments in chimeric reads. (B) MdoR-target 482 interactions found in Hfq CLASH, RIL-seq S-chimera data (log and stationary phase) and RNase E 483 CLASH data. (C) MdoR forms stable duplexes with the 5'UTR of porin-encoding mRNAs and the OhsC sRNA. In silico prediction (RNAcofold (Lorenz et al., 2011)) of hybrid structures derived from the most abundant MdoR chimeric reads with the indicated transcripts. The min. MFE is the minimum folding energy assigned by RNAcofold. (D) Pulse-overexpression of MdoR using L-arabinose. The empty pBAD plasmid served as a negative control. Samples were harvested 15-minutes after induction. (E) MdoR regulates expression of various mRNAs. DESeq2 analyses were performed on RNA-seq data from three biological replicates. Red points indicate differentially expressed transcripts. Transcripts with a $\log _{2}$ fold-change $>0$ were enriched in the Control data; those with a $\log _{2}$ fold-change $<0$ were enriched in the MdoR overexpression data. The annotated, enlarged red data points indicate several differentially expressed transcripts discussed in the text. $(F)$ The MdoR seed region is important for target regulation. RT-qPCR analysis of several differentially expressed transcripts (gene names shown at the top of the plots), in the presence of sRNAs. The plasmid-borne sRNAs were induced for 15 minutes using Larabinose. The sRNA names are indicated below each bar; the 'no sRNA' sample contains the empty plasmid as reference for fold-change calculations; recA was used as the internal reference gene; experiments were performed in technical triplicates; the standard error of the mean (SEM) of two biological replicates are reported as error bars.

499

Intriguingly, MdoR overexpression also reduced the levels of sRNAs RyeA and MicA, the latter of which depends on $\sigma^{\mathrm{E}}$ for its expression (Udekwu and Wagner, 2007). In Salmonella, MicA downregulates LamB, a high affinity maltose/maltodextrin transporter (Bossi and Figueroa-Bossi, 2007). Fragments of three mRNAs (ompC, ompA and $p t s H$ ) that were found in MdoR chimeric reads were also differentially expressed in the MdoR overexpression RNA-seq data, providing strong evidence that these are direct MdoR targets.

All the available data suggest that MdoR is part of a mixed coherent feed forward regulatory network (FFL) that enhances the uptake of maltose/maltodextrin by maltose 508 transporters (see Discussion). By base-pairing with the 5'UTRs of the mRNAs it firstly reduces 509 the flux of the more general porins such as OmpC and OmpA to the outer membrane. 510 Secondly, we propose that MdoR stimulates the accumulation of the high-affinity maltose porin 511 LamB in the OMP by suppressing the inhibitory $\sigma^{\mathrm{E}}$ pathway and MicA. To further test this 512 model, we performed additional validation experiments. First, we confirmed the DESeq2 513 results for a number of the regulated genes (ompC, rpoE, micA and rye A) by RT-qPCR (Figure 514 7F). We included an MdoR mutant in which seed sequence in stem 1 was changed into its 515 complementary sequence (Figure 8-figure supplement $1 \mathrm{~A}$ ). As control we also included the 516 RybB sRNA, which regulates rpoE and ompC expression (Gogol et al., 2011; Papenfort et al., 517 2006; Thompson et al., 2007). In all cases, the MdoR SM mutations reduced the negative 
regulatory effect on target expression (Figure 7F).

To demonstrate direct target regulation in vivo, we employed a well-established reporter system where an sRNA is co-expressed with a construct containing the mRNA target region fused to the coding sequence of superfolder green fluorescent protein (sfGFP) (Corcoran et al., 2012; Urban and Vogel, 2007) (Figure 8-figure supplement 1B). Fusions for ompC, ompA and $\sigma^{\mathrm{E}}$ were constructed, but only the OmpC and OmpA-sfGFP reporters produced stable fusions that could be analysed. We also included an MdoR sRNA seed sequence mutant (MdoR SM) and an ompC mutant containing compensatory mutations (OmpC SM; Figure 8-figure supplement 1A). Unfortunately, our OmpA-GFP reporter construct that contained compensatory mutations in the target region did not generate a stable fusion. Therefore, we were unable to use this reporter system to verify the MdoR-ompA interaction. As positive controls we used the MicC and RybB sRNAs as they both regulate E. coli ompC expression (Chen et al., 2004; Gogol et al., 2011). Fluorescence measurements confirmed that levels of OmpC-sfGFP and OmpA-sfGFP fusions were significantly lower in cells expressing MdoR (Figure 8-figure supplement 1C). Importantly, MdoR overexpression did not change the expression of the GFP reporter itself (Figure 8-figure supplement $1 \mathrm{C}$ ). Mutating the MdoR seed region largely restored OmpA- and OmpC-sfGFP reporter levels to the levels of the no sRNA negative control. The MdoR SM mutant was still able to partially suppress the expression of the OmpC SM-sfGFP mutant, suggesting that base-pairing interactions between these two mutants is less stable compared to the wild-type (Figure 8-figure supplement $1 \mathrm{C}$ ). The wild-type MdoR was also able to partially suppress the expression of the ompC SM mutant. We suggest that the predicted base-pairing interactions between MdoR and ompC in the second stem (Figure 8-figure supplement 1A) might be sufficient to partially suppress ompC expression. Regardless, the data strongly imply that MdoR directly regulates ompC expression.

To determine whether the changes in fluorescence signal correlate with changes in reporter mRNA levels, we measured the expression of the GFP reporters by RT-qPCR. The results were essentially identical to the GFP fluorescence measurements (Figure 8A); Overexpression of the wild-type MdoR, but not seed mutant, reduced ompC-sfGFP mRNA levels. The ompC seed mutation (SM) did not fully disrupt regulation by wild-type MdoR. However, the MdoR mutant containing compensatory mutations (SM mutant) was able to much better suppress the ompC SM mRNA levels, consistent with the idea that base-pairing was (largely) restored. Next, we performed polysome profiling experiments to assess the level of ompC translation upon overexpression of MdoR. Although MdoR overexpression did not noticeably affect $70 \mathrm{~S}$ and polysome levels (Figure 8-figure supplement $2 \mathrm{~A}$ ), we observed a significant $(\sim 37 \%)$ reduction of ompC mRNA in the polysomal fractions, relative to the upper fractions (Figure 8-figure supplement 2B). We conclude that MdoR can regulate ompC 
A

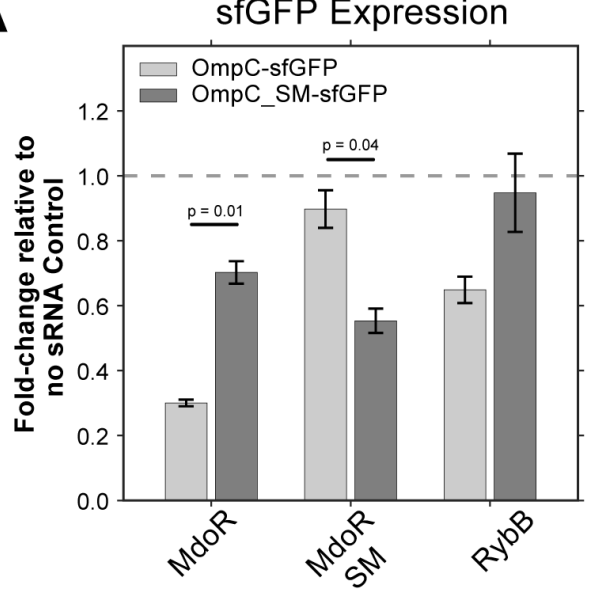

B

C
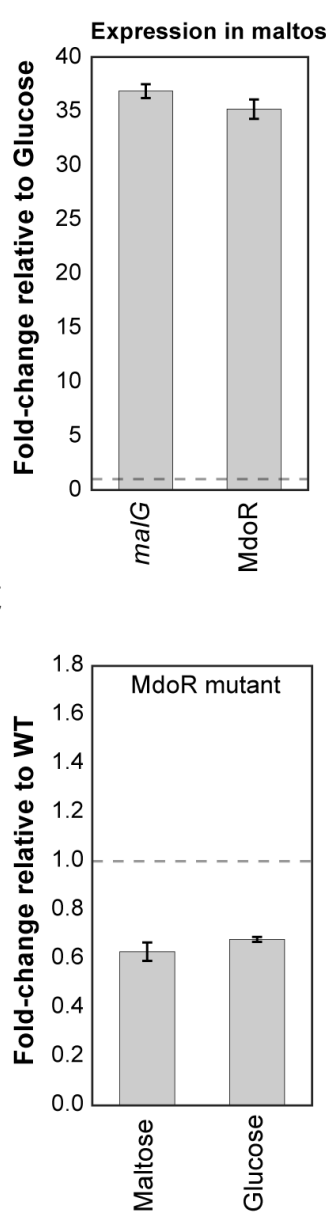

D

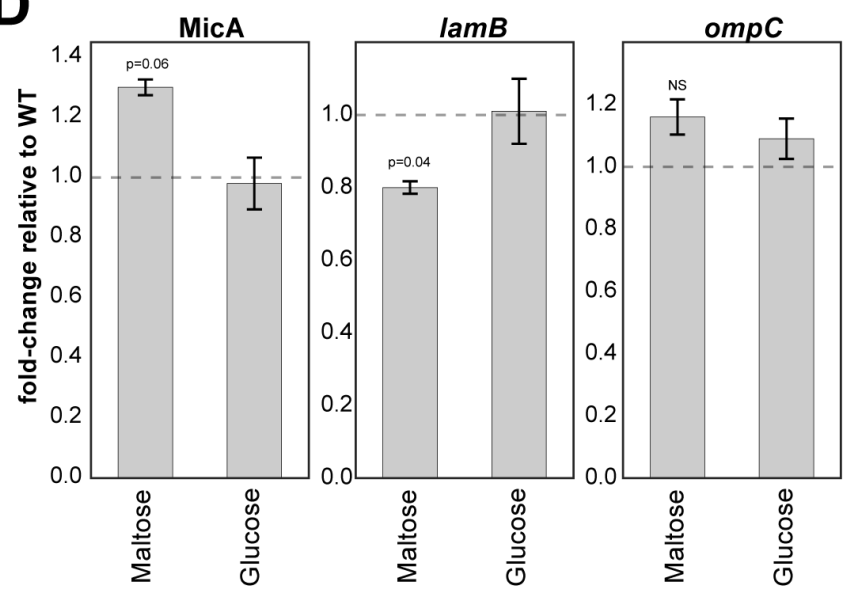

$\mathbf{E}$
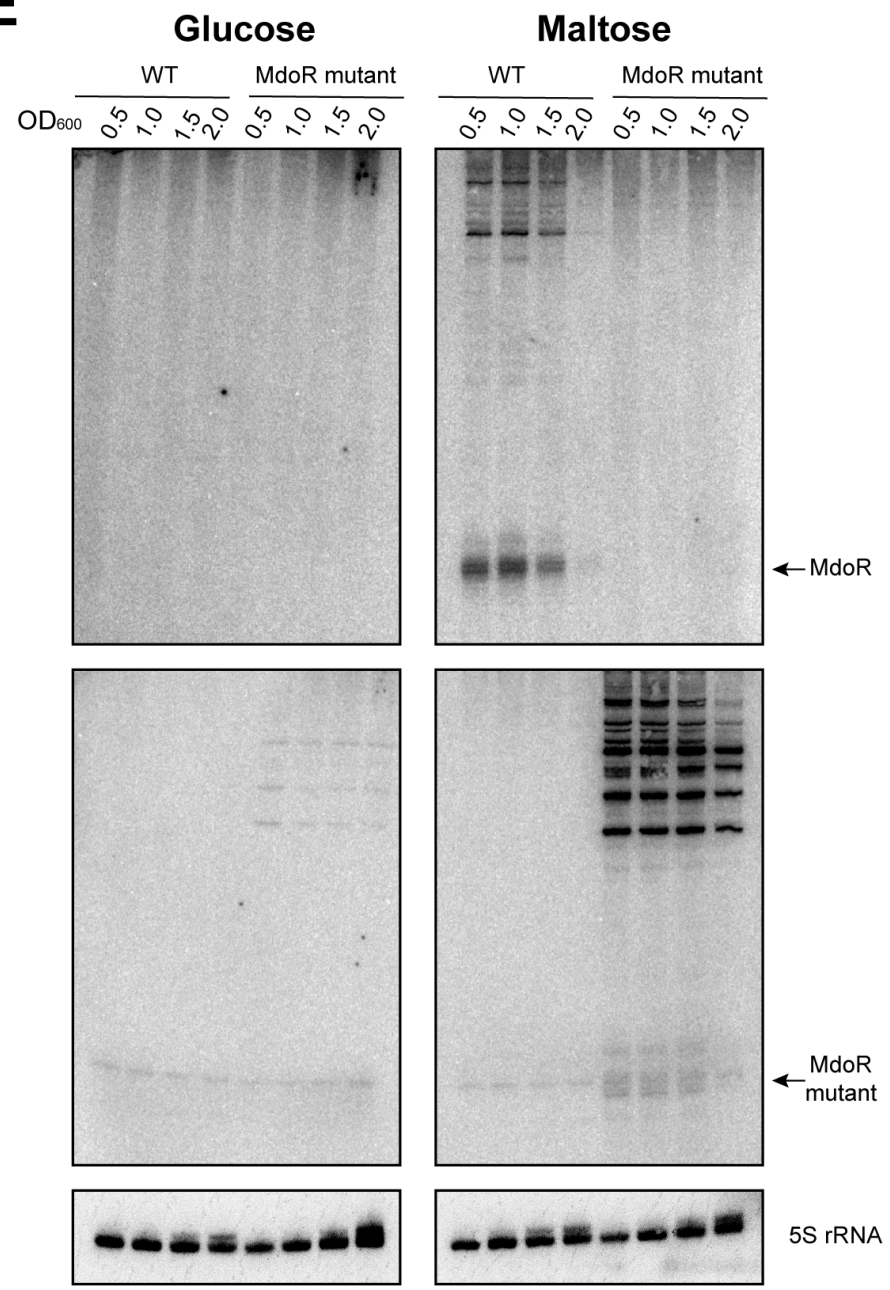

Figure 8. MdoR modulates expression of key factors involved in maltose intake.

560 (A) MdoR directly downregulates ompC mRNA through base-pairing interactions: RT-qPCR analyses 561 of the ompC-sfGFP and ompC SM-sfGFP fusions expression in the presence of MdoR. The bars 562 indicate the mean fold-change in expression relative to the no sRNA Control (horizontal dashed line). 
563 Error bars indicate the standard error of the mean from two biological replicates. The significance of the

564 differences between the WT and MdoR SM was assessed with a two-tailed Student's t-test. (B)

565 Endogenous MdoR and malG expression is induced during growth on maltose, and ompC levels are 566 significantly lower in maltose compared to glucose. Total RNA extracted from exponentially growing 567 cells $\left(\mathrm{OD}_{600} 0.5\right)$ and MdoR, malG (Left), and MdoR direct (ompC) and indirect (MicA) targets was 568 quantified by RT-qPCR. The data were normalized to $5 \mathrm{~S}$ rRNA levels. The bars indicate the mean fold569 change in expression relative to expression in cells growing in glucose (indicated on the plot with a 570 horizontal dashed line). Error bars indicate the standard error of the mean from two biological replicates.

571 (C) The mutant MdoR is $\sim 50 \%$ less abundant than the wild-type. Cell growth and RT-qPCR analysis 572 of MdoR and malG expression was performed as in (b). The bars indicate the mean fold-change in 573 expression relative to the wild-type. Error bars indicate the standard error of the mean from two 574 biological replicates. (D) Increased MicA and decreased lamB levels in the MdoR seed mutant. P-values 575 were calculated using with a one-sample t-test. (E) MdoR seed sequences are important for RNase E 576 recruitment and MdoR biogenesis. Northern blot that compares MdoR and longer malG-3'UTR 577 containing fragments expression in wild-type E. coli and the MdoR seed mutant strain.

578

\section{MdoR enhances maltoporin expression during maltose fermentation}

580

581

To further substantiate these results, we next switched to a more controlled system to investigate the effect of endogenous MdoR on its targets. To determine whether MdoR has a role in adaptation to maltose-metabolising conditions, single overnight cultures grown in glucose were split and (re)inoculated in fresh medium containing either glucose or maltose as the sole carbon sources, and expression of several mal regulon genes and MdoR targets were quantified. We show that MdoR and its parental transcript ma/G are almost undetectable during growth in glucose, and highly expressed during growth in maltose ( 35 -fold increase, Figure $8 \mathrm{~B}$ ). This is consistent with catabolite repression of the mal regulon by glucose, and its induction my maltose (Boos and Shuman, 1998). Intriguingly, we observed that ompC mRNA levels are overall significantly lower during growth in maltose, compared to glucose (Figure $8 \mathrm{~B})$. This suggests that porin expression is also regulated by nutrient source in $E$. coli. Similarly, MicA, a repressor of LamB synthesis, has reduced levels in maltose compared to glucose (Figure 8B). We next mutated the entire seed sequence of the chromosomal copy of MdoR (both stem 1 and 2; Figure 8-figure supplement $1 \mathrm{~A}$ ) in the chromosomal context, to completely disrupt base-pairing with ompC mRNA. Notably, the fully-processed mutant MdoR sRNA is less abundant than the wild-type (Figure 9C) and longer (unprocessed) fragments that contain upstream malG regions could be readily detected (Figure $9 \mathrm{E}$ ). We speculate that the mutation in the upstream MdoR sequence might have affected RNase E recruitment or cleavage, impairing MdoR processing. The MdoR mutant strain also accumulated significantly higher levels of MicA (Figure 8D) and less lamB. Levels of ompC mRNA levels were also 
600

601

602

603

604

605

606

607

608

609

610

611

612

613

614

615

616

617

618

619

620

621

622

623

624

625

626

627

628

629

630

631

632

633

634

635

slightly elevated in the mutant in maltose relative to the parental strain, although in this case a similar increase was also observed in glucose.

Collectively, the data suggest a role for MdoR in enhancing the uptake of maltose when more favourable carbon sources become limiting.

\section{Discussion}

Microorganisms need to constantly adapt their transcriptional program to counteract changes in their environment, such as changes in temperature, cell density and nutrient availability. In bacteria, small RNAs (sRNAs) and their associated RNA-binding proteins are believed to play a key role in this process. By controlling translation and degradation rates of mRNAs upon stress imposition (Holmqvist and Wagner, 2017; Nitzan et al., 2017; Shimoni et al., 2007), they can regulate the kinetics of gene expression as well as suppress noisy signals (Beisel and Storz, 2011), enabling organisms to more efficiently adapt to environmental changes. A major challenge for bacteria is the transition from exponential growth to stationary phase, when the most favourable nutrients become limiting. To counteract this challenge, cells need to rapidly remodel their transcriptome to be able to efficiently metabolize alternative carbon sources. This transition is very dynamic and involves activation and repression of diverse metabolic pathways. However, it is unclear to what degree sRNAs contribute to this transition. The most useful piece of information would be to know what sRNAs are upregulated during this transition phase and to identify their RNA targets. This would help to uncover the regulatory networks that govern this adaptation, as well as provide a starting point for more detailed functional analyses on sRNAs predicted to play a key role in this process. For this purpose, we performed UV cross-linking, ligation and sequencing of hybrids (CLASH (Kudla et al., 2011)) to unravel the sRNA-target interactions during this transition. Using $\mathrm{Hfq}$ as a bait we uncovered thousands of unique sRNA-target interactions. Our data are consistent with previously published work (Melamed et al., 2016; Waters et al., 2016) but we also identified almost 1700 novel sRNA-mRNA interactions and over 100 novel sRNA-sRNA interactions. We experimentally validated several of the interactions found in our CLASH findings. We identified functional sRNA-sRNA interactions and describe a novel 3'UTR derived SRNA that plays a role in enhancing uptake of an alternative carbon source during the transition to stationary phase. 
A
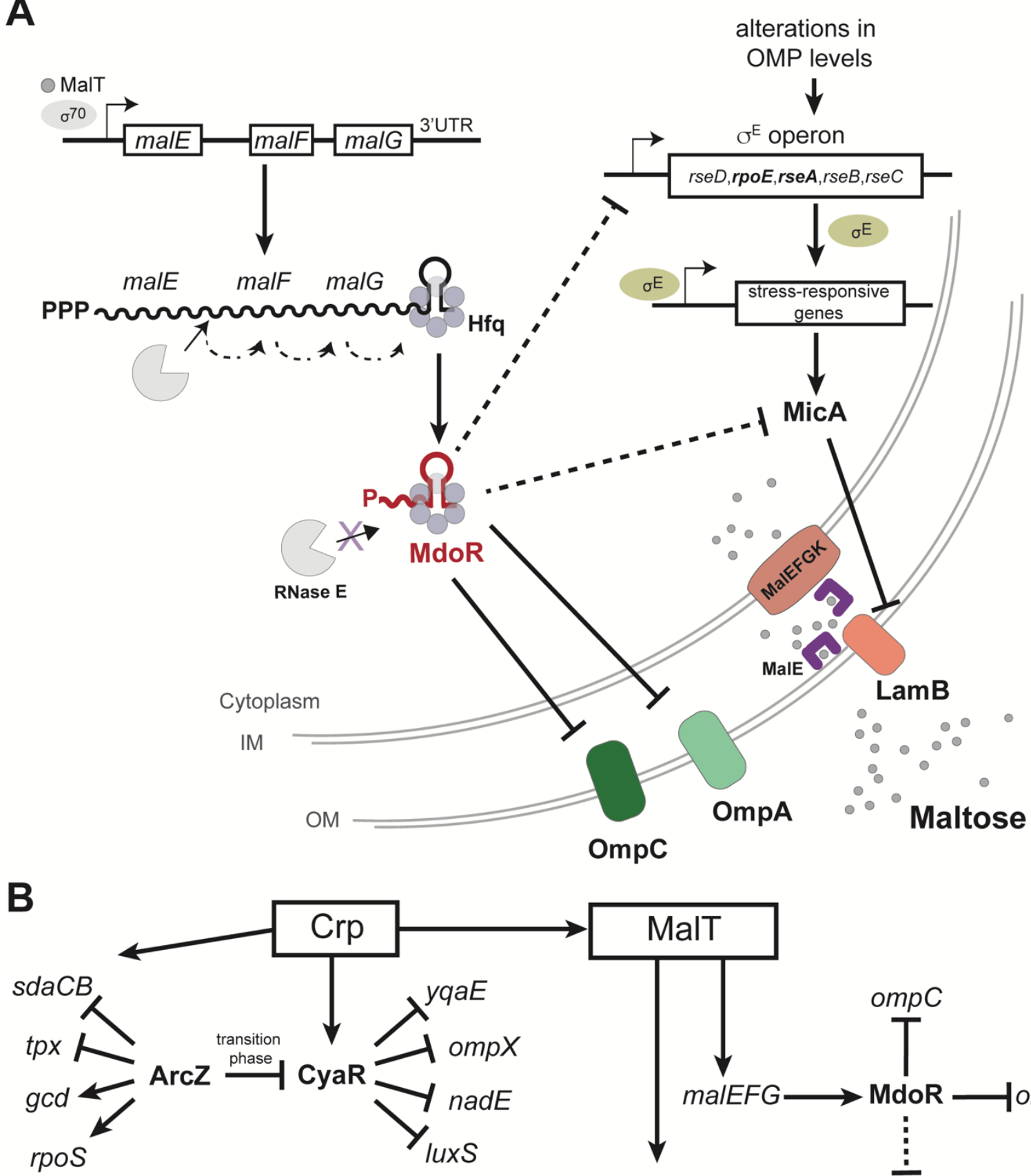

Figure 9. CLASH uncovers sRNA-target interaction networks that regulate adaptation to changes in nutrient availability.

639 (A) Model for MdoR biogenesis and its role in post-transcriptional regulation. During maltose (grey circles) utilization, MalT transcribes the malEFG operon that encodes components for maltose uptake. A fraction of the malEFG transcripts is degraded by RNase E, generating the 3'UTR-derived MdoR sRNA. MdoR base-pairs with ompC mRNA, leading to its decay. Additionally, MdoR downregulates expression of ompA and MicA that represses LamB synthesis. Therefore, as part of a feed-forward loop 
644 (FFL), MdoR promotes accumulation of maltose specific porins, which facilitates maltodextrin uptake.

645 Increased production of LamB exerts additional pressure on the OM assembly machinery. This

646 extracytoplasmic stress, is suppressed by $\sigma^{\mathrm{E}}$ activation, that controls the expression of stress-

647 responsive genes and most of the assembly and insertion factors. But $\sigma^{E}$ activation is not desirable in

648 these conditions for two reasons: its unbalanced activation has precarious effects on the cell, and would

649 produce high amounts of MicA, that would ultimately repress LamB production. For these reasons,

650 MdoR regulation includes a regulatory arm that mitigates excessive $\sigma^{\mathrm{E}}$ response through MdoR

651 repression of $r p o E$ expression and prevention of $\sigma^{\mathrm{E}}$ activation. The latter is achieved by downregulation

652 of omp mRNAs, which indirectly relieves envelope stress. (B) sRNA-target networks regulating 653 peptide/amino acids and maltose/maltodextrin uptake. Boxed are the key transcription factors, sRNAs 654 are in bold and italicized names are mRNA targets. CRP-cAMP induces expression of CyaR, which is

655 repressed by $A r c Z$, specifically during the transition phase, a regulatory circuit that connects multiple 656 pathways related to the onset of stationary phase and biofilm formation to quorum sensing, cellular 657 adherence and the nutritional state of the cell. MalT transcribes lamB and, via malEFG transcription, 658 promotes MdoR accumulation. MdoR indirectly promotes LamB synthesis by repressing the opposing $659 \sigma^{\mathrm{E}}$ pathway and its sRNA, MicA. Thus, MalT and MdoR jointly promote lamB expression, forming a 660 mixed coherent FFL.

661

662

\section{Hfq CLASH}

Our S. cerevisiae Cross-linking and cDNA analysis data (CRAC; (Granneman et al., 2009)) showed that a percentage of the cDNAs were formed by intermolecular ligations of two RNA fragments (chimeras) known to base pair in vivo (Kudla et al., 2011). These findings prompted us to develop a refined protocol to enrich for sRNA-target chimeric reads using $\mathrm{Hfq}$ as an obvious bait. The initial Hfq UV cross-linking data (CRAC; (Tree et al., 2014)) did not yield sufficiently high numbers of chimeric reads to extract new biological insights. In line with observations from other groups (Bandyra et al., 2012; Bruce et al., 2018; Morita et al., 2005), it was proposed that duplexes formed by $\mathrm{Hfq}$ are rapidly transferred to the RNA degradosome

671 (Bandyra et al., 2012; Bruce et al., 2018; Morita et al., 2005). This can cause an extensive reduction in the likelihood of capturing sRNA-target interactions with $\mathrm{Hfq}$ using CLASH (Waters et al., 2016). However, a recent study demonstrated that Hfq can be used effectively as a bait to enrich for sRNA-target duplexes under lower-stringency purification conditions suggesting that sRNA-mRNA duplexes are sufficiently stable on $\mathrm{Hfq}$ during purification (Melamed et al., 2016). This encouraged us to further optimize the CLASH method. We made a number changes to the protocol that, when combined, enabled us to recover a large number of sRNA-target chimeric reads (detailed in Materials and Methods). We shortened various incubation steps to minimize RNA degradation and performed very long and stringent washes after bead incubation steps to remove background binding of non-specific proteins and RNAs. 
linked RNAs prior to making cDNA libraries, ensuring the recovery of longer chimeric RNA fragments. The resulting cDNA libraries were paired-end sequenced to increase the recovery of chimeric reads with high mapping scores from the raw sequencing data. These modifications led to a substantial improvement in the recovery of chimeric reads $(9.5 \%$ compared to $0.001 \% .0 .71 \%$ were intermolecular chimeras).

Both RIL-seq and Hfq CLASH have advantages and disadvantages, but they are highly complementary approaches. A major strength of $\mathrm{CLASH}$, however, is that the purification steps are performed under highly stringent and denaturing conditions. During the first FLAG affinity purification steps the beads are extensively washed with high salt buffers and the second Nickel affinity purification step is done under completely denaturing conditions (6M guanidium hydrochloride). These stringent purification steps enable can significantly reduce noise by strongly enriching for RNAs covalently cross-linked to the bait protein (Granneman et al., 2009). Indeed, we show that Hfq CLASH can generate high quality RNA-RNA interaction data with very low background: Only a few hundred chimeric reads were found in control datasets, compared to the over 70.000 that co-purified with Hfq. The RIL-seq library preparation protocol uses an rRNA depletion step to remove contaminating ribosomal RNA, whereas for Hfq CLASH this is not necessary. Our library preparation protocol also includes the use of random nucleotides in adapter sequences to remove potential PCR duplicates (“collapsing") from the data.

The very stringent purification conditions used in CLASH could, in some cases also be a disadvantage as it completely relies on UV cross-linking to isolate directly bound RNAs. In cases where protein-RNA cross-linking efficiencies are low (for example proteins that only recognize double-stranded RNA), RIL-seq may be a better approach as it does not completely rely on UV cross-linking (Melamed et al., 2016).

A large number of interactions were unique to both RIL-seq and Hfq CLASH datasets, which we believe can be explained by a number of technical and experimental factors. The denaturing purification conditions used with CLASH completely disrupts the Hfq hexamer ((Tree et al., 2014) and this work) and therefore during the adapter ligation reactions the RNA ends are likely more accessible for ligation. In support of this, in the RIL-Seq data, the sRNAs are mostly found in the second half of the chimeras (Melamed et al., 2016), in the Hfq CLASH data we see sRNAs fragments roughly equally distributed in both sides (45\% in left fragment and $55 \%$ in right fragment). Indeed, it was proposed that in RIL-seq the 3 ' end of the SRNA is buried in the hexamer and therefore not always accessible for ligation (Melamed et al., 2016).

For the RIL-seq experiments, the authors harvested the cells at $4^{\circ} \mathrm{C}$ and they resuspend the cells in ice-cold PBS prior to UV irradiation (Melamed et al., 2018, 2016), which results in a cold-shock that can affect the sRNA-interactome as well as sRNA stability. We cross-link actively growing cells in their growth medium and we UV irradiate our cells only for 
several seconds using the Vari-X-linker we recently developed (van Nues et al., 2017). We use filtration devices to rapidly harvest our cells (less than 30 seconds) and the filtered cells are subsequently stored at $-80^{\circ} \mathrm{C}$. We previously showed that filtration combined with short UV cross-linking times dramatically reduces noise introduced by the activation of the DNA damage response and significantly increased the recovery of short-lived RNA species (van Nues et al., 2017). We speculate that many of the interactions that are unique to our Hfq CLASH data represent short-lived RNA duplexes that are preferentially captured with our UV cross-linking and rapid cell filtration setup.

\section{The 3'UTR derived sRNA MdoR functions in a mixed feed forward loop by suppressing opposing pathways}

We found a large number of chimeras that represent over a hundred distinct intermolecular interactions between 3'UTRs and other mRNA regions, which implicate direct mRNA-mRNA communication. These interactions could have been formed by 3'UTR fragments that have been processed by RNase $E$ (or other RNases) or by an sRNA located within the 3'UTR that transcribed from an internal promoter. These 3'UTR fragments are primarily described as decoys or sponges for other sRNAs (Miyakoshi et al., 2015b), but could act as trans-acting sRNAs as well (Chao and Vogel, 2016). Among the interactions in our data we found 79 interactions between 3'UTRs and 5'UTRs (Supplementary Table 2.6). We speculate that many of these represent novel 3'UTR-derived sRNAs that target 5'UTRs of mRNAs. Reanalysis of RNA-seq data from a study that globally mapped $E$. coli transcription start sites (TSS) (Thomason et al., 2015) identified 47 TSS in these 3'UTR, strongly suggesting that these 3'UTRs host sRNAs. The majority of these 3'UTR fragments appear to have 5' monophosphates. We also verified some of our findings by Northern blot analyses. Thus, we have potentially uncovered many novel 3'UTR-derived sRNAs.

Some of these predictions were validated by our follow up work (discussed below) and, while this work was in progress, also by others (Melamed et al., 2016). One of the 3'UTRderived SRNAs we uncovered (MdoR) was of particular interest as it is only detected during the transition from late exponential to early stationary phase. A model of how MdoR contributes to maltose uptake is shown in Figure 9A.

The genetic structure and transcriptional regulation of the mal regulon are well understood. However, its post-transcriptional regulation has remained largely unexplored. Our work uncovered new links between the maltose uptake ( $m a l)$ regulon, envelope stressresponses and membrane composition/assembly pathways. While initially cells metabolize more favourable carbon sources such as glucose, these are generally rapidly depleted and bacteria need to quickly switch to alternative carbon sources, such as maltose and maltodextrins. During maltose utilisation, the malEFG operon is transcribed by the MalT 
transcription factor and the transcript is processed by RNase $E$ as well as other degradosome components (Khemici and Carpousis, 2004). Here we show that the MdoR sRNA is a product of malEFG processing, which is protected from further degradation by $\mathrm{Hfq}$.

MdoR is unique in a sense that it not only a 3'UTR derived SRNA that targets multiple pathways, but also because it is part of a mixed coherent feed forward loop (FFL) that we predict promotes maltose uptake via LamB. Efficient uptake of maltose/maltodextrin not only requires the inner membrane transporters encoded by the malEFG operon, but also the highaffinity maltose transporter LamB (Figure 9A), which cooperates with the inner membrane proteins to import these carbon sources. $\operatorname{lamB}$ is significantly upregulated when cells start to utilize alternative carbon sources. A major advantage of a coherent FFL is that it can accelerate or delay responses to stimuli, such as changes in carbon source availability. In this FFL, the key activator is the MalT transcription factor, which induces the expression of both malEFG and lamB when cells start to consume maltose (Figure 9B). Expression of new OMPs, however, needs to be carefully coordinated as any changes in the protein composition of the $\mathrm{OM}$, such as increased levels of LamB (or accumulation of misfolded LamB in the periplasm), can lead to induction of the $\sigma^{E}$ envelope stress response caused by the reorganisation of the membrane when maltodextrins are utilised (Figure 9A)(Kenyon et al., 2005). We propose that malEFG indirectly promotes LamB accumulation via the MdoR sRNA that (a) reduces the levels of general porins (OmpC and $\mathrm{OmpA})$ and (b), likely indirectly, dampens the $\sigma^{\mathrm{E}}$ stress response as a result of OMP level reduction. Dampening $\sigma^{\mathrm{E}}$ expression important during maltose uptake as it induces the expression of MicA, which directly downregulates lamB translation (Bossi and Figueroa-Bossi, 2007). When ectopically expressed at high levels, MdoR reduces mRNA levels of ompC and $o m p A$, which may free up the resources enabling efficient accumulation of $\mathrm{LamB}$ in the outer membrane. At physiological levels MdoR significantly reduces MicA expression and we observed a $\sim 20 \%$ increase in lamB mRNA levels. OmpC mRNA levels were not substantially affected. While these changes appear modest, it is important to take into consideration the very high abundance and intrinsic stability of lamB and ompC mRNAs (minute-long half-lives). It is therefore expected that even a mild reduction in their mRNA levels can profoundly relieve the pressure on the OMP translation and assembly pathways (Guo et al., 2014). The net outcome of mal regulon transcription, MdoR biogenesis and regulatory activity, is increased expression of high-affinity components of maltose-specific transport (MalE and LamB).

MdoR is reminiscent of the Vibrio cholerae MicX sRNA that also mapped to the 3' region of malG and regulates levels of outer membrane proteins (Davis and Waldor, 2007).

790 However, the MicX targets are different from MdoR. Furthermore, unlike MdoR, MicX is 
792

793

794

795

796

797

798

799

800

801

802

803

804

805

806

807

808

809

810

811

812

813

814

815

816

817

818

819

820

821

822

823

824

825

826

827

upstream Mal operon in V. cholerae do not affect accumulation of MicX (Davis and Waldor, 2007), whereas the levels of MdoR strongly correlate with malEFG mRNA levels. Nevertheless, both studies demonstrate that an sRNA resides in the 3'UTR of the malG transcript and pinpoint a mechanism where the expression of multiple porins is regulated by sRNAs encoded within transporter mRNAs.

\section{CLASH reveals large sRNA-sRNA interaction networks.}

Our analyses unearthed an unexpectedly large number $(>100)$ of sRNA-sRNA interactions. The majority of these interactions involved known sRNA seed sequences, suggesting that these could represent bona fide interactions that prevent sRNAs from basepairing with their targets or result in the degradation of the sRNAs. About 40 sRNA-sRNA interactions involving sRNAs from the core genome were detected in the RNase E CLASH dataset, about a quarter of which were also detected in our Hfq data. The relatively low overlap between the datasets is not surprising, given the differences in the growth conditions (virulence inducing conditions for enterohaemorrhagic $E$. coli vs growth transitions for commensal E. coli MG1655). Moreover, many of the sRNA-sRNA interactions recovered in association with RNase $E$ are likely duplexes in the process of being degraded. Many of the sRNA-sRNA interactions unique to our Hfq data may represent interactions between antisRNAs and seed sequences of sRNAs which may not necessarily involve recruitment/activity of RNase $E$.

The chimeras containing CyaR fragments were of particular interest as CyaR is preferentially expressed during the transition from late exponential to stationary phase (De Lay and Gottesman, 2009; Wassarman et al., 2001) and may therefore play an important role in adaptation to nutrient availability. We could show that overexpression of ArcZ, which basepairs with CyaR in our CLASH data, significantly reduced CyaR levels (Figure 9B). Using seed mutants and compensatory mutations, we confirmed direct interactions between ArcZ and CyaR and our data suggests that this interaction specifically takes place during the transition phase. Interestingly in Salmonella, overexpression of ArcZ showed a dramatic reduction in CyaR bound to Hfq and upregulation of CyaR targets, such as nadE (Papenfort et al., 2009), suggesting that this activity is conserved between these two Gram-negative bacteria. A similar type of asymmetric regulation has also been elegantly demonstrated for the Qrr3 sRNA of Vibrio cholera (Feng et al., 2015). The fate of these sRNA-sRNA duplexes may depend on the position of the interaction; It was shown that if sRNA-target RNA base-pair within a stabilizing 5' stem, the sRNA will be preferentially degraded (Feng et al., 2015). Consistent with this, folding of the chimeric reads suggests that ArcZ preferentially base-pairs with CyaR at the 5' end (Figure 4), which may alter secondary structures that normally help to stabilize the sRNA. 
828 The biological role of ArcZ targeting CyaR is unclear, however, a possible function could be

829 to reduce noise in CyaR expression by preventing CyaR levels from overshooting during the 830 transition phase.

831 ArcZ and CyaR target mRNAs are associated with many different processes. Thus, 832 these interactions are expected to connect multiple pathways. For example, ArcZ regulation 833 of CyaR may connect adaptation to stationary phase/biofilm development (De Lay and 834 Gottesman, 2009; Monteiro et al., 2012) to quorum sensing and cellular adherence (De Lay 835 and Gottesman, 2009). CyaR expression is controlled by the global regulator Crp. Most of the 836 genes controlled by Crp are involved in transport and/or catabolism of amino acids or sugar. 837 Interestingly, ArcZ downregulates the sdaCB dicistron which encodes for proteins involved in 838 serine uptake and metabolism (Papenfort et al., 2009). This operon has been shown to be 839 regulated by Crp as well, suggesting that ArcZ can counteract the activity of Crp.

\section{Materials and Methods}

842

\section{Bacterial strains and culture conditions}

An overview of the bacterial strains used in this study is provided in Supplementary Table 5. The E. coli MG1655, TOP10 or TOP10F' strains served as parental strains. The $E$. coli K12 strain used for CLASH experiments, MG1655 hfq::HTF was previously reported (Tree under aerobic conditions with shaking at $200 \mathrm{rpm}$. The media were supplemented with antibiotics where required at the following concentrations: ampicillin (Sigma, UK, A9518) - 100 $\mu \mathrm{g} / \mathrm{ml}$, chloramphenicol (Corning, US, C239RI) - $25 \mu \mathrm{g} / \mathrm{ml}$, kanamycin (Gibco, US, 11815-024) - $50 \mu \mathrm{g} / \mathrm{ml}$. Where indicated, $0.2 \%$ glucose or maltose were used. For induction of sRNA expression from plasmids, $1 \mathrm{mM}$ IPTG, $200 \mathrm{nM}$ anhydrotetracycline hydrochloride (Sigma, 1035708-25MG) or 0.2\% L-arabinose (Sigma, A3256) were used.

\section{Construction of sRNA expression plasmids}

For the pulse-overexpression constructs, the sRNA gene of interest was cloned the transcriptional +1 site under Para control by amplifying the pBAD+1 plasmid (Supplementary

859 Table 5) by inverse PCR using Q5 DNA Polymerase (NEB). The pBAD+1 template is derived 860 from pBADmycHisA (Tree et al., 2014). The sRNA genes and seed mutants (SM) were 861 synthesized as ultramers (IDT; Supplementary Table 5), which served as the forward primers, 862 as described (Tree et al., 2014). The reverse primer (oligo pBAD+1_5P_rev) bears a 863 monophosphorylated 5'-end to allow blunt-end self-ligation. The PCR reaction was digested 
linear PCR product was circularized by self-ligation, performed as above. Ligations were transformed in DH5a competent cells. Positive transformants were screened by sequencing. The control plasmid $p B A D+1$ was constructed similarly by self-ligation of the PCR product generated from oligonucleotides pBAD+1_Xbal_fwd and pBAD+1_5P_rev. Small RNA overexpression constructs derived from the pZA21MCS and pZE12/uc (Expressys) were generated identically, using the indicated ultramers in Supplementary Table 5 as forward primers, and oligos pZA21MCS_5P_rev and pZE12_5P_rev as reverse primers, respectively, and transformed in E. coli TOP10F'.

\section{Construction of mRNA-superfolder GFP fusions}

Supplementary Table 5 lists all the plasmids, gene fragments and primers used for cloning procedures in this work. To construct constitutively expressed, in-frame mRNA-sfGFP fusions for the fluorescence reporter studies, the 5'UTR, start codon and first $\sim 5$ codons of target genes were cloned under the control of PLtetO-1 promoter in a PXG10-SF backbone as previously described (Corcoran et al., 2012; Urban and Vogel, 2007). Derivatives of the target-GFP fusion plasmids harbouring seed mutations (SM) were generated using synthetic mutated gene-fragments (IDT, Belgium, Supplementary Table 5). To prepare the inserts, the target region of mRNA of interest was either amplified by PCR from E. coli genomic DNA or synthesized as g-blocks (IDT, Belgium) and cloned using Nhel and Nsil restriction sites. Transformants were screened by restriction digest analysis and verified by Sanger sequencing (Edinburgh Genomics, Edinburgh, UK).

\section{Hfq UV Cross-linking, Ligation and Analysis of Hybrids (Hfq-CLASH)}

CLASH was performed essentially as described(Waters et al., 2016), with a number of modifications including changes in incubation steps, cDNA library preparation, reaction volumes and UV cross-linking. E. coli expressing the chromosomal Hfq-HTF were grown overnight in $\mathrm{LB}$ at $37^{\circ} \mathrm{C}$ with shaking (200 rpm), diluted to starter $\mathrm{OD}_{600} 0.05$ in fresh $\mathrm{LB}$, and re-grown with shaking at $37^{\circ} \mathrm{C}$ in $750 \mathrm{ml} \mathrm{LB}$. A volume of culture equivalent to $80 \mathrm{OD}_{600}$ per $\mathrm{ml}$ was removed at the following cell-densities $\left(\mathrm{OD}_{600}\right): 0.4,0.8,1.2,1.8,2.4,3.0$ and 4.0 , and immediately subjected to UV $(254 \mathrm{~nm})$ irradiation for 22 seconds $(\sim 500 \mathrm{~mJ} / \mathrm{cm} 2)$ in the VariX-linker (van Nues et al., 2017) (https://www.vari-x-link.com). Cells were harvested using a rapid filtration device (van Nues et al., 2017) (https://www.vari-x-link.com) onto $0.45 \mu \mathrm{M}$ nitrocellulose filters (Sigma, UK, HAWP14250) and flash-frozen on the membrane in liquid nitrogen. Membranes were washed with $\sim 15 \mathrm{ml}$ ice-cold phosphate-buffered saline (PBS), and cells were harvested by centrifugation. Cell pellets were lysed by bead-beating in 1 volume per weight TN150 buffer (50mM Tris pH 8.0, $150 \mathrm{mM} \mathrm{NaCl}, 0.1 \% \mathrm{NP}-40,5 \mathrm{mM} \beta$ mercaptoethanol) in the presence of protease inhibitors (Roche, A32965), and 3 volumes 0.1 
902

903

904

905

906

907

908

909

910

911

912

913

914

915

916

917

918

919

920

921

922

923

924

925

926

927

928

929

930

931

932

933

934

935

936

937

$\mathrm{mm}$ Zirconia beads (Thistle Scientific, 11079101z), by performing 5 cycles of 1 minute vortexing followed by 1-minute incubation on ice. One additional volume of TN150 buffer was added. To reduce the viscosity of the lysate and remove contaminating DNA the lysate was incubated with RQ1 DNase I (10U/ml Promega, M6101) for 30 minutes on ice. Two-additional volumes of TN150 were added and mixed with the lysates by vortexing. The lysates were centrifuged for 20 minutes at $4000 \mathrm{rpm}$ at $4^{\circ} \mathrm{C}$ and subsequently clarified by a second centrifugation step at $13.4 \mathrm{krpm}$, for $20 \mathrm{~min}$ at $4^{\circ} \mathrm{C}$. Purification of the UV cross-linked HfqHTF-RNA complexes and cDNA library preparation was performed as described (Granneman et al., 2009). Cell lysates were incubated with $50 \mu \mathrm{l}$ of pre-equilibrated M2 anti-FLAG beads (Sigma, M8823-5ML) for $1-2$ hours at $4^{\circ} \mathrm{C}$. The anti-FLAG beads were washed three times 10 minutes with $2 \mathrm{ml}$ TN1000 (50 mM Tris pH 7.5, 0.1\% NP-40, $1 \mathrm{M} \mathrm{NaCl}$ ) and three times 10 minutes with TN150 without protease inhibitors (50 mM Tris pH 7.5, 0.1\% NP-40, 150mM $\mathrm{NaCl}$ ). For TEV cleavage, the beads were resuspended in $250 \mu \mathrm{l}$ of TN150 buffer (without protease inhibitors) and incubated with home-made GST-TEV protease at room temperature for 1.5 hours. The TEV eluates were then incubated with a fresh 1:100 dilution preparation of RNacelt (RNase A and T1 mixture; Agilent, 400720) for exactly 5 minutes at $37^{\circ} \mathrm{C}$, after which they were mixed with $0.4 \mathrm{~g} \mathrm{GuHCl}(6 \mathrm{M}$, Sigma, G3272-100G), $\mathrm{NaCl}(300 \mathrm{mM})$, and Imidazole (10mM, I202-25G). Note this needs to be carefully optimized to obtain high-quality cDNA libraries. The samples were then transferred to $50 \mu \mathrm{l}$ Nickel-NTA agarose beads (Qiagen, 30210), equilibrated with wash buffer 1 (6 M GuHCl, 0.1\% NP-40, $300 \mathrm{mM} \mathrm{NaCl}, 50 \mathrm{mM}$ Tris $\mathrm{pH} 7.8,10 \mathrm{mM}$ Imidazole, $5 \mathrm{mM}$ beta-mercaptoethanol). Binding was performed at $4^{\circ} \mathrm{C}$ overnight with rotation. The following day, the beads were transferred to Pierce SnapCap spin columns (Thermo Fisher, 69725), washed 3 times with wash buffer 1 and 3 times with 1xPNK buffer (10 mM MgCl $2,50 \mathrm{mM}$ Tris $\mathrm{pH} 7.8,0.1 \% \mathrm{NP}-40,5 \mathrm{mM}$ beta-mercaptoethanol). The washes were followed by on-column TSAP incubation (Thermosensitive alkaline phosphatase, Promega, M9910) treatment for $1 \mathrm{~h}$ at $37^{\circ} \mathrm{C}$ with $8 \mathrm{U}$ of phosphatase in $60 \mu \mathrm{l}$ of $1 \mathrm{xPNK}$, in the presence of $80 \mathrm{U}$ RNasin (Promega, N2115). The beads were washed once with $500 \mu \mathrm{l}$ wash buffer 1 and three times with $500 \mu \mathrm{l}$ 1xPNK buffer. To add 3'-linkers (AppPE - Supplementary Table 5), the Nickel-NTA beads were incubated in $80 \mu \mathrm{l}$ 3'-linker ligation mix with (1 X PNK buffer, 1 MM 3'-adapter, 10\% PEG8000, 30U Truncated T4 RNA ligase 2 K227Q (NEB, M0351L), 60U RNasin). The samples were incubated for 4 hours at $25^{\circ} \mathrm{C}$. The 5 '-ends of bound RNAs were radiolabelled with $30 \mathrm{U}$ T4 PNK (NEB, M0201L) and $3 \mu \mathrm{l}^{32} \mathrm{P}$ YATP $\left(1.1 \mu \mathrm{Ci}\right.$; Perkin Elmer, NEG502Z-500) in 1 XPNK buffer for 40 min at $37^{\circ} \mathrm{C}$, after which ATP (Roche, 11140965001) was added to a final concentration of $1 \mathrm{mM}$, and the incubation prolonged for another 20 min to complete 5'-end phosphorylation. The resin was washed three times with $500 \mu \mathrm{l}$ wash buffer 1 and three times with equal volume of 1 XPNK buffer. For on- 
bead $5^{\prime}$-linker ligation, the beads were incubated $16 \mathrm{~h}$ at $16^{\circ} \mathrm{C}$ in $1 \times P N K$ buffer with $40 \mathrm{U}$ T4 RNA ligase I (NEB, M0204L), and $1 \mu 100 \mu \mathrm{M}$ L5 adapter (Supplementary Table 5), in the presence of $1 \mathrm{mM}$ ATP and 60U RNasin. The Nickel-NTA beads were washed three times with wash buffer 1 and three times with buffer 2 (50 mM Tris- $\mathrm{HCl} \mathrm{pH} \mathrm{7.8,50} \mathrm{mM} \mathrm{NaCl,} 10 \mathrm{mM}$ imidazole, $0.1 \%$ NP-40, $5 \mathrm{mM} \beta$-mercaptoethanol). The protein-RNA complexes were eluted in two steps in new tubes with $200 \mu \mathrm{l}$ of elution buffer (wash buffer 2 with $250 \mathrm{mM}$ imidazole). The protein-RNA complexes were precipitated on ice by adding TCA (T0699-100ML) to a final washed with $800 \mu \mathrm{l}$ acetone, and air dried for a few minutes in the hood. The protein pellet was resuspended and incubated at $65^{\circ} \mathrm{C}$ in $20 \mu \mathrm{l} 1 \mathrm{x}$ NuPage loading buffer (Thermo Scientific, NP0007), resolved on 4-12\% NuPAGE gels (Thermo Scientific, NP0323PK2) and visualised by autoradiography. The cross-linked proteins-RNA were cut directly from the gel and incubated with $160 \mu \mathrm{g}$ of Proteinase $\mathrm{K}$ (Roche, 3115801001) in $600 \mu \mathrm{l}$ wash buffer 2 subsequently extracted by phenol-chloroform extraction and ethanol precipitated. The RNA pellet was directly resuspended in RT buffer and was transcribed in a single reaction with the SuperScript IV system (Invitrogen, 18090010) according to manufacturer's instructions using the PE_reverse oligo as primer. The cDNA was purified with the DNA Clean and Concentrator 5 kit (Zymo Research) and eluted in $11 \mu$ DEPC water. Half of the cDNA (5 $\mu$ l) was amplified by PCR using Pfu Polymerase (Promega, M7745) with the cycling conditions $\left(95^{\circ} \mathrm{C}\right.$ for 2 min; 20-24 cycles: $95^{\circ} \mathrm{C}$ for $20 \mathrm{~s}, 52^{\circ} \mathrm{C}$ for $30 \mathrm{~s}$ and $72^{\circ} \mathrm{C}$ for $1 \mathrm{~min}$; final extension of $72^{\circ} \mathrm{C}$ for $5 \mathrm{~min}$ ). Libraries were resolved on a $2 \%$ MetaPhor agarose (Lonza, LZ50181) gel and 175-300bp fragments were gel-extracted with the MinElute kit (Qiagen, 28004) according to manufacturer's instructions. All libraries were quantified on a 2100 Bionalyzer using the HighSensitivity DNA assay and a Qubit 4 (Thermo Scientific, Q33226). Individual libraries were pooled based on concentration and barcode sequence identity. Paired-end sequencing (75 bp) was performed by Edinburgh Genomics on an Illumina HiSeq 4000 platform.

\section{RNA-seq}

E. coli MG1655 was cultured, UV-irradiated and harvested as described for the CLASH procedure. Total RNA was extracted using the Guanidium thiocyanate phenol method. RNA

972 integrity was assessed with the Prokaryote Total RNA Nano assay on a 2100 Bioanalyzer 973 (Agilent, G2939BA). Sequencing libraries from two biological replicates were prepared by 
974 NovoGene using the TruSeq library preparation protocol and 150bp paired-end sequencing

975 was performed on an Illumina NovaSeq 6000 system. This yielded $\sim 7-8$ million paired-end

976 reads per sample. For the overexpression analysis of MdoR, we generated RNA-seq libraries

977 using an in-house protocol. Genomic DNA was removed by incubating $10 \mu \mathrm{g}$ of total RNA with

$9782 \mathrm{U}$ Turbo DNase (Thermo Scientific, AM2238) in a $50 \mu \mathrm{l}$ final volume for 30 minutes at $37^{\circ} \mathrm{C}$

979 in the presence of $10 \mathrm{U}$ Superaseln RNase Inhibitor (Thermo Scientific, AM2694). RNA was

980 subsequently phenol-chloroform extracted and purified by ethanol-precipitation. Ribosomal

981 RNA was removed with the Ribo-Zero rRNA Removal Kit (Gram-Negative Bacteria; Illumina,

982 MRZGN126) according to the manufacturer's instructions. Successful rRNA depletion was

983 verified on the Agilent 2100 Bioanalyzer. The RNA was fragmented for 5 min at $95^{\circ} \mathrm{C}$ in the

984 presence of Superscript III buffer (Invitrogen) followed by a five-minute incubation on ice.

985 Reverse-transcription (RT) was performed with Superscript III (Thermo Scientific, 18080044)

986 in $20 \mu \mathrm{l}$ reactions according to manufacturer's procedures using $250 \mathrm{ng}$ of ribosomal RNA

987 depleted RNA and $2.5 \mu \mathrm{M}$ random hexamers (PE_solexa_hexamer, oligo 73, Supplementary

988 Table 5). The RNA and free primers were degraded using $20 \mathrm{U}$ of Exonuclease I (NEB,

$989 \mathrm{M} 0293 \mathrm{~L}$ ) and 50U RNaself (NEB, M0243S) and the cDNA was purified with the DNA Clean \&

990 Concentrator 5 kit (Zymo Research). Ligation of the 5' adapter (P5_phospho_adapter, oligo

99139 ) to the cDNA was performed using CircLigase II (Lucigen, CL9021K) for 6 hours at $60^{\circ} \mathrm{C}$,

992 followed by a 10 -minute inactivation at $80^{\circ} \mathrm{C}$. The cDNA was purified with the DNA Clean \&

993 Concentrator 5 kit. Half of the cDNA library was PCR amplified using Pfu polymerase

994 (Promega, M7745) using the P5 forward PCR oligonucleotide and barcoded BC reverse

995 oligonucleotides (200 nM; Supplementary Table $5 ; 95^{\circ} \mathrm{C}$ for $2 \mathrm{~min}, 95^{\circ} \mathrm{C}$ for $20 \mathrm{~s}, 52^{\circ} \mathrm{C}$ for $30 \mathrm{~s}$

996 and $72^{\circ} \mathrm{C}$ for $1 \mathrm{~min}$, and a final extension of $72^{\circ} \mathrm{C}$ for $5 \mathrm{~min}$. 20 cycles of amplification). The

997 PCR products were treated with Exonuclease 1 (NEB, M0293L) for $1 \mathrm{~h}$ at $37^{\circ} \mathrm{C}$ and purified

998 by ethanol precipitation. Libraries were resolved on a 2\% MetaPhor agarose gel 200-500 bp

999 fragments were gel-extracted using the MinElute kit. All libraries were quantified on a 2100

1000 Bionalyzer using the High-Sensitivity DNA assay (Agilent, 5067-4627). Individual libraries

1001 were pooled in equimolar amounts. Paired-end sequencing (75 bp) was performed by

1002 Edinburgh Genomics on the Illumina HiSeq 4000 platform.

1003

\section{Small RNA overexpression studies}

1005 Individual TOP10F' clones carrying pZA21 and pZE12-derived sRNA constructs and 1006 control plasmids combinations (Supplementary Table 5) were cultured to $O_{600} 0.1$ and 1007 expression of sRNAs was induced with IPTG and anhydrotetracycline hydrochloride (Sigma, 1008 I6758-1G and 1035708-25MG) for one hour. Cells were collected by centrifugation for 30 1009 seconds at $14000 \mathrm{rpm}$, flash-frozen in liquid nitrogen and total RNA was isolated as above. 
1010 Genomic DNA was digested with Turbo DNase (Thermo Scientific, AM2238), then the RNA was purified with RNAClean XP beads (Beckman Coulter, A63987). Gene expression was quantified by RT-qPCR (see below) using $10 \mathrm{ng}$ total RNA as template, and expressed as fold change relative to the reference sample containing pJV300 (Sittka et al., 2007) or pZA21.

For pulse-overexpression studies overnight MG1655 cultures containing pBAD::sRNA and empty $\mathrm{pBAD}+1$ control plasmids were inoculated in fresh LB-ampicillin medium at a starting $\mathrm{OD}_{600}$ of 0.05 and grown aerobically at $37^{\circ} \mathrm{C}$ to $\mathrm{OD}_{600} 0.4$. Pre-induction $(0 \mathrm{~min})$ and post-induction samples were harvested. For induction, cultures were supplemented with Larabinose (Sigma, A3256) and rapidly collected by filtration and flash-frozen in liquid nitrogen at the indicated time-points. RNA was extracted from three biological replicate time-series, followed by RNA-seq library preparation, next generation sequencing and DESeq2 analysis of differentially expressed genes.

\section{GFP reporter system to quantify sRNA effect on target expression}

A two-plasmid system was used to express each sRNA, and mRNA-sfGFP fusions (Corcoran et al., 2012; Urban and Vogel, 2007) with modifications. The sRNA and sfGFPfusion plasmids were co-transformed in E. coli TOP10 cells by electroporation and cells were maintained on dual selection with ampicillin and chloramphenicol. In TOP10 cells, the mRNAsfGFP constructs are constitutively expressed, whereas sRNA expression requires Larabinose induction. The expression of sfGFP-fused targets in the presence or absence of sRNAs was quantified at the protein level, by plate reader experiments and at the RNA level, by RT-qPCR.

For the plate reader experiments, a single colony of bacterial strain harbouring a sRNA-target-sfGFP combination was inoculated in a 96-well Flat Bottom Transparent Polystyrene plate with lid (Thermo Scientific, 152038) and cultured overnight at $37^{\circ} \mathrm{C}$ in 100 $\mu$ LB supplemented with antibiotics and L-arabinose (Sigma, A3256) to induce expression of sRNAs. Next day, each overnight inoculum was diluted 1:100 by serial dilution, in triplicate, in LB with freshly prepared L-arabinose to a final volume of $100 \mu \mathrm{l}$. Cultures were grown in a 96well plate in an Infinite 200 Pro plate reader (Tecan) controlled by i-control software (Tecan) for 192 cycles at $37^{\circ} \mathrm{C}$ with $1 \mathrm{~min}$ orbital shaking ( $4 \mathrm{~mm}$ amplitude) every 5 th minute. To monitor optical density over time, the following parameters were used: wavelength $600 \mathrm{~nm}$, bandwidth $9 \mathrm{~nm}$. Fluorescence was monitored with excitation wavelength $480 \mathrm{~nm}$, bandwidth

$10429 \mathrm{~nm}$ and emission wavelength $520 \mathrm{~nm}$, bandwidth $20 \mathrm{~nm}$. Measurements were recorded at 1043 5-minute intervals, by top reading. Raw data was processed following guidance from previous 1044 reports (Urban and Vogel, 2007). First, the range of linearity of increase of fluorescence with $1045 \mathrm{OD}_{600}$ was identified for all individual triplicates. Only the linearity range common to all 
triplicates was considered for further analysis. For each set of triplicates, the mean fluorescence was calculated at each $\mathrm{OD}_{600}$. To correct for background and cell autofluorescence, the mean fluorescence of a strain with plasmid pXG-0 was subtracted from all strains with GFP plasmids at the equivalent $\mathrm{OD}_{600}$. Ultimately, a curve was generated for each sample, plotting the background-corrected fluorescence (GFP) versus $\mathrm{OD}_{600}$. The experiments were performed for three biological replicates, and mean values and standard error of the means calculated for each strain.

1053

\section{RT-qPCR}

Total RNA $(12.5 \mu \mathrm{g})$ was treated with $2 \mathrm{U}$ of Turbo DNase (Thermo Scientific, AM2238) for 1 hour at $37^{\circ} \mathrm{C}$ in a $10 \mu$ reaction in the presence of $2 \mathrm{U}$ Superaseln RNase inhibitor (Thermo Scientific, AM2694). The DNase was inactivated by 10 minutes incubation at $75^{\circ} \mathrm{C}$. Reverse transcription (RT) was performed in a single reaction for all target genes of interest using a mix of gene-specific RT primers at $3.5 \mu \mathrm{M}$ concentration each. After addition of $2.5 \mu \mathrm{l}$ RT primer mix, the RNA and primers were denatured at $70^{\circ} \mathrm{C}$ for $3 \mathrm{~min}$, then snap chilled and incubated on ice for $5 \mathrm{~min}$. RT was performed for 1 hour at $55^{\circ} \mathrm{C}$ with SuperScript III (Thermo Scientific, 18080051) using $5 \mu \mathrm{l}$ of RNA-RT primers mix in $10 \mu \mathrm{l}$ final volume (100 U Superscript III, $2.5 \mathrm{mM}$ DTT, 1xFS Buffer, $0.75 \mathrm{mM}$ dNTPs) in the presence of $1 \mathrm{U}$ RNasin (Promega, N2115). RT was followed by treatment with $5 \mathrm{U}$ RNase $\mathrm{H}$ for 30 min at $37^{\circ} \mathrm{C}$ to remove the RNA from the RNA-cDNA duplexes. The cDNA was diluted 10-fold with DEPC water. Quantitative PCR was performed on 50ng of DNAse I-treated total RNA using the Brilliant III UltraFast SYBR Green QPCR Master Mix (Agilent, \#600883) and the Luna Universal One-Step RT-qPCR Kit (NEB, E3005E) according to manufacturer's instructions. The qPCRs were run on a LightCycler 480 (Roche), and the specificity of the product was assessed by generating melting curves, as follows: $65^{\circ} \mathrm{C}-60 \mathrm{~s}, 95^{\circ} \mathrm{C}(0.11$ ramp rate with 5 acquisitions per ${ }^{\circ} \mathrm{C}$, continuous). The data analyses were performed with the IDEAS2.0 software, at default settings: Absolute Quantification/Fit Points for Cp determination and Melt Curve Genotyping.

1074 The qPCR efficiency of primer pairs was assessed by performing standard curves by serial dilution of template RNA or genomic DNA. Negative controls such as -RT or no template control were used throughout, and the qPCR for all samples was performed in technical triplicate. Outliers from the samples with technical triplicate standard deviations of $\mathrm{Cp}>0.3$ were discarded from the analyses. To calculate the fold-change relative to the control, the $2^{-}$ ddCp method was employed, using $\operatorname{recA}$ or $5 \mathrm{~S}$ rRNA ( $r r f D)$ as the reference genes where indicated. Experiments were performed for minimum two biological replicates, and the mean 
significance of the fold-change difference compared to the reference sample control (for which fold-change $=1$ ) was tested with a one-sample t-test.

\section{Northern Blot analysis}

1086

Total RNA was extracted from cell lysates by GTC-Phenol extraction. For large RNA fragments, $10 \mu \mathrm{g}$ of total RNA was resolved on a $1.25 \%$ BPTE- gel $(\mathrm{pH} 7)$ and transferred to a nylon membrane (HyBond N+, GEHealthcare, RPN1210B) by capillarity. For short RNA fragments, $10 \mu \mathrm{g}$ total RNA was separated on an $8 \%$ polyacrylamide TBE-Urea gel and transferred to a nitrocellulose membrane by electroblotting for four hours at $50 \mathrm{~V}$. Membranes were pre-hybridised in $10 \mathrm{ml}$ of UltraHyb Oligo Hyb (Thermo Scientific, AM8663) for one hour and probed with ${ }^{32} \mathrm{P}$-labeled DNA oligo at $42^{\circ} \mathrm{C}$ for $12-18$ hours in a hybridization oven. The sequences of the probes used for Northern blot detection are detailed in Supplementary Table 5. Membranes were washed twice with $2 \times S S C+0.5 \%$ SDS solution for 10 minutes and visualized using a Phosphor imaging screen and FujiFilm FLA-5100 Scanner (IP-S mode). For detection of highly abundant species (5S rRNA) autoradiography was used for exposure.

\section{Western blot analyses}

A fraction of the E. coli MG1655 Hfq::htf lysates used for the RNA-seq experiments using strains cultured, cross-linked, harvested and lysed in identical conditions as the CLASH experiments containing $40 \mu \mathrm{g}$ protein was run on PAGE gels and transferred to a nitrocellulose membrane. The membranes were blocked for one hour in blocking solution ( $5 \%$ non- fat milk in PBST (1X phosphate saline buffer, 0.1\% Tween-20). To detect Hfq-HTF protein, the membrane was probed overnight at $4^{\circ} \mathrm{C}$ with the Rabbit anti-TAP polyclonal primary antibody (Thermo Fisher, 1:5000 dilution in blocking solution), which recognizes an epitope at the region between the TEV-cleavage site and His6. For the loading control we used a rabbit polyclonal to GroEL primary antibody (Abcam, 1:150000 dilution, ab82592), for 2 hours at room temperature. After 3×10 min PBST washes, the membranes were blotted for one hour with a Goat anti-rabbit IgG H\&L (IRDye 800) secondary antibody (Abcam, ab216773, 1:10000 in blocking solution) at room temperature. Finally, after three 10-minute PBST washes, the blot was rinsed in PBS, and the proteins were visualised with a LI-COR (Odyssey CLX) using the $800 \mathrm{~nm}$ channel and scan intensity 4. Image acquisition and quantifications were performed with the Image Studio Software.

\section{Primer extension analysis}

1116 One microgram total RNA was reverse-transcribed using SuperScript III reverse 1117 transcriptase (Thermo Scientific, 18080051) using ${ }^{32} \mathrm{P}$-radiolabelled oligonucleotides as 
1118

1119

1120

1121

1122

1123

1124

1125

1126

1127

1128

1129

1130

1131

1132

1133

1134

1135

1136

1137

1138

1139

1140

1141

1142

1143

1144

1145

1146

1147

1148

1149

1150

1151

1152

1153

primers (Supplementary Table 5). Primers were added to the RNA and annealing was performed by heating the samples at $85^{\circ} \mathrm{C}$ for three minutes and then snap chilling them on ice. The RT was performed for one hour at $45^{\circ} \mathrm{C}$, followed by Exonuclease I and RNaself (NEB M0293L and M0243S) $\left(0.5 \mu \mathrm{l}\right.$ each) treatment for 30 minutes at $37^{\circ} \mathrm{C}$. Reactions were stopped by mixing with an equal volume of 2XRNA loading dye (NEB, B0363S), 2 minutes incubation at $95^{\circ} \mathrm{C}$ and snap chilled. The sequencing ladders were prepared with Sequenase v2.0 (Thermo Scientific, 70775Y200UN) according to specified instructions. Samples were resolved on 6\% PAA/8M TBE-urea gels and visualized using the FujiFilm FLA5100 scanner.

\section{Construction of the MdoR seed-mutant strain}

To mutate the chromosomal copy of MdoR, we used the $\lambda$ Red system (Datsenko and Wanner, 2000). We amplified the integration cassette from plasmid pKD4 with ultramers 895 and 896, containing homology regions to the coding sequence of malG, the desired MdoR sequence and to the region immediately downstream of the Rho-independent terminator, respectively. With this design, the scar after removal of the Kan cassette was expected at a site outside the MdoR/malG sequence. The PCR product was electroporated in $E$. coli MG1566 strains carrying the pKD46 plasmid from which $\lambda$ Red recombinase was induced with $10 \mathrm{mM}$ L-arabinose. Correct replacement of the MdoR seed sequence was screened by colony PCR using primer pairs: $725 \& 909$ and $726 \& 910$ (Supplementary Table 5). The antibiotic resistance cassette was removed from substitution mutants by FLP-recombinase expressed constitutively from pE-FLP (St-Pierre et al., 2013). Successful allele replacement was confirmed by Sanger sequencing.

\section{Polysome profiling analyses}

Wild-type E. coli MG1655 containing empty PBAD plasmid and an isogenic strain containing pBAD::MdoR were grown in $L B$ until $O_{600} 0.4$, then treated for 15 minutes with $L-$ arabinose (Sigma, A3256) to induce overexpression of MdoR, and cycloheximide (Sigma, C4859-1ML) at a final concentration of $100 \mu \mathrm{g} / \mathrm{ml}$ for 3 minutes. $200 \mathrm{ml}$ of cells were harvested by rapid filtration and flash frozen. The cells were washed in ice-cold PBS supplemented with $100 \mu \mathrm{g} / \mathrm{ml}$ cycloheximide.

Polysomal profiling was performed according to previously described protocols (Bernabò et al., 2017; Lunelli et al., 2016) with minor changes in the lysis buffer $(10 \mathrm{mM} \mathrm{NaCl}$, $10 \mathrm{mM} \mathrm{MgCl}_{2}, 10 \mathrm{mM}$ Tris-HCl (pH 7.5), $100 \mu \mathrm{g} / \mathrm{ml}$ cycloheximide, 1\% (w/v) Na-Deoxycholate, 1U RQ DNAse I (Promega, M6101), 0.6U/mL RiboLock (Thermo Scientific, EO0381) in DEPC water). Lysates were kept on ice for $30 \mathrm{~min}$, centrifuged $3 \mathrm{X}$ at $15000 \mathrm{~g}$ for $10 \mathrm{~min}$. The supernatants were loaded on a linear $10 \%-30 \%[\mathrm{w} / \mathrm{v}]$ sucrose gradient and centrifuged for 4 
hours using a SW41 rotor at $40000 \mathrm{rpm}$ in a Beckman Optima XPN-100 Ultracentrifuge.

1155 Fractions of $1 \mathrm{~mL}$ in volume were collected monitoring the absorbance at $254 \mathrm{~nm}$ with the UA-

11566 UV/VIS detector (Teledyne Isco). Fractions from the entire gradient (total RNA) and from the

1157 fractions corresponding to ribosomes (70S) and polysomes (polysomal RNA) were pooled

1158 and RNA was purified by acid phenol-chloroform extraction according to (Tebaldi et al., 2012).

\section{Terminator $^{\mathrm{TM}}$ 5'-PhosphateDependent Exonuclease treatment}

Ten micrograms of total RNA extracted from cell-samples at $\mathrm{OD}_{600} 1.2$ and 1.8 were treated with 5'-Terminator Dependent Exonuclease (Lucigen, TER51020) as per manufacturer instructions using Buffer $A$. The reaction was terminated by phenol extraction and ethanol precipitation, and the RNA was loaded on $8 \%$ polyacrylamide-urea gels and transferred to nylon membranes that were probed for MdoR, CpxQ, RybB and 5S rRNA (Supplementary Table 5).

\section{Seed mutant studies}

Wild-type MG1655 and seed mutant strains were grown overnight in minimal medium with glucose. Next day, each starter culture was split and inoculated at $\mathrm{OD}_{600} 0.05$ in fresh $M 9$ medium with glucose or maltose as the sole carbon source. Growth was monitored and cells were harvested at $\mathrm{OD}_{600} 0.5$. Total RNA was extracted, and gene expression was quantified by RT-qPCR or Northern Blot.

\section{Computational analysis}

1176 Pre-processing of the raw sequencing data.

1177 Raw sequencing reads in fastq files were processed using a pipeline developed by

1178 Sander Granneman, which uses tools from the pyCRAC package (Webb et al., 2014). The entire pipeline is available at https://bitbucket.org/sgrann/). The CRAC_pipeline_PE.py pipeline first demultiplexes the data using pyBarcodeFilter.py and the in-read barcode sequences found in the L5 5' adapters. Flexbar then trims the reads to remove 3'-adapter sequences and poor-quality nucleotides (Phred score <23). Using the random nucleotide information present in the L5 5'-adaptor sequences, the reads are then collapsed to remove potential PCR duplicates. The reads were then mapped to the E. coli MG1655 genome using Novoalign (www.novocraft.com). To determine to which genes the reads mapped to, we generated an annotation file in the Gene Transfer Format (GTF). This file contains the start and end positions of each gene on the chromosome as well as what genomic features (i.e. sRNA, protein- coding, tRNA) it belongs to. To generate this file, we used the Rockhopper software (Tjaden, 2015) on E. coli rRNA-depleted total RNA-seq data (generated by Christel 
1191

1192

1193

1194

1195

1196

1197

1198

1199

1200

1201

1202

1203

1204

1205

1206

1207

1208

1209

1210

1211

1212

1213

1214

1215

1216

1217

1218

1219

1220

1221

1222

1223

1224

1225

1226

1227

resulting GTF file contained information not only on the coding sequences, but also complete 5' and 3' UTR coordinates. We then used pyReadCounters.py with Novoalign output files as input and the GTF annotation file to count the total number of unique cDNAs that mapped to each gene.

\section{Normalization steps.}

To normalise the read count data generated with pyReadCounters.py and to correct for differences in library depth between time-points, we calculated Transcripts Per Million reads (TPM) for each gene. Briefly, for each time-point the raw counts for each gene was first divided by the gene length and then divided by the sum of all the values for the genes in that time-point to normalize for differences in library depth. The TPM values for each $\mathrm{OD}_{600}$ studied were divided by the TPM values of the first sample $\left(\mathrm{OD}_{600} 0.4\right)$ and were then $\log _{2}$-normalized. The $\log _{2}$-normalized fold-changes were used to compare RNA-seq and Hfq-cross-linking profiles among samples, and to perform k-means clustering with the python sklearn. cluster.KMeans class.

\section{Hfq-binding coverage plots.}

For the analysis of the Hfq binding sites the pyCRAC package (Webb et al., 2014) was used (versions. 1.3.2-1.4.4). The pyBinCollector tool was used to generate Hfq cross-linking distribution plots over genomic features. First, PyCalculateFDRs.py was used to identify the significantly enriched Hfq-binding peaks (minimum 10 reads, minimum 20 nucleotide intervals). Next, pyBinCollector was used to normalize gene lengths by dividing their sequences into 100 bins and calculate nucleotide densities for each bin. To generate the distribution profile for all genes individually, we normalized the total number of read clusters (assemblies of overlapping cDNA sequences) covering each nucleotide position by the total number of clusters that cover the gene. Motif searches were performed with pyMotif.py using the significantly enriched Hfq-binding peaks (FDR intervals). The 4-8 nucleotide k-mers with Z-scores above the indicated threshold were used for making the motif logo with the k-mer probability logo tool (Wu and Bartel, 2017) with the -ranked option (http://kplogo.wi.mit.edu/).

\section{Analysis of chimeric reads.}

Chimeric reads were identified using the hyb package using default settings (Travis et al., 2013) and further analysed using the pyCRAC package (Webb et al., 2014). To apply this single-end specific pipeline to our paired-end sequencing data, we joined forward and reverse reads using FLASH (Magoč and Salzberg, 2011), which merges overlapping paired reads into a single read. These, as well as any remaining single reads, were then analysed using hyb. The -anti option for the hyb pipeline was used to be able to use a genomic $E$. coli hyb database, 
rather than a transcript database. Uniquely annotated hybrids (.ua.hyb) were used in subsequent analyses. To visualise the hybrids in the genome browser, the .ua.hyb output files were converted to the GTF format. To generate distribution plots for the genes to which the chimeric reads mapped, the parts of the chimeras were clustered with pyClusterReads.py and BEDtools (Quinlan and Hall, 2010) (intersectBed) was used to remove clusters that map to multiple regions. To produce the coverage plots with pyBinCollector, each cluster was counted only once, and the number of reads belonging to each cluster was ignored.

sRNA density plots.

To visualize the nucleotide read density of sRNA-target pairs for a given SRNA, we first merged the hyb datasets for all $\mathrm{OD}_{600}$ and biological replicates and filtered the interactions that were found statistically significant in the unified dataset. For each sRNA-target pair in the filtered dataset, the hit counts at each nucleotide position for all chimeras were summed. The count data was $\log _{2}$-normalised (actually $\log _{2}($ Chimera count +1 ) to avoid $\mathrm{NaN}$ for nucleotide positions with 0 hits when log-transforming the data).

\section{sRNA-sRNA network visualization.}

Only the sRNA-sRNA chimeric reads representing statistically significant interactions in the merged CLASH dataset were considered. For each such interaction, chimera counts corresponding in either orientation were summed, $\log _{2}$-transformed and visualized with the igraph Python package.

\section{Differential expression analyses.}

For the differential expression analyses DESeq2 was used (Love et al., 2014). Three MdoR pulse-overexpression datasets were compared to three pBAD Control overexpression datasets. Only differentially expressed genes that had an adjusted p-value of 0.05 or lower were considered significant.

\section{Multiple sequence alignments and conservation analyses.}

The homologous sequences of MdoR in other enterobacteria were retrieved by BLAST. JalView was used for the multiple sequence alignments, using the MAFFT algorithm (Waterhouse et al., 2009).

\section{Data and Code availability.}

The next generation sequencing data have been deposited on the NCBI Gene Expression Omnibus (GEO) with accession number GSE123050. The python pyCRAC(Webb et al., 2014), kinetic-CRAC and GenomeBrowser software packages used for analysing the 
data are available from https://bitbucket.org/sgrann (pyCRAC up to version 1.3.3), https://git.ecdf.ed.ac.uk/sgrannem/ and pypi (https://pypi.org/user/g ronimo/). The hyb pipeline for identifying chimeric reads is available from https://github.com/gkudla/hyb. The

1268 FLASH algorithm for merging paired reads is available from 1269 https://github.com/dstreett/FLASH2. Bedgraph and Gene Transfer Format (GTF) generated 1270 from the analysis of the Hfq CLASH, RNA-seq and TEX RNA-seq data(Thomason et al., 2015) 1271 are available from the Granneman lab DataShare repository

1272 (https://datashare.is.ed.ac.uk/handle/10283/2915).

1273

Acknowledgement

We are grateful to Lionello Bossi and Meriem El Karoui for their valuable feedback on the project and fruitful discussions. We thank Christel Sirocchi for help with preparing E. coli RNA-seq libraries, Erica de Leau for expert technical assistance and the members of the Granneman lab for critically reading the manuscript. We thank Jean-Marie Pages and Julia Vergalli for help the analyses of LamB levels and for fruitful discussions. This work was supported by grants from the Wellcome Trust (091549 to S.G and 102334 to I.A.I.), the Wellcome Trust Centre for Cell Biology core grant (092076), a Medical Research Council non Clinical Senior Research Fellowship (MR/R008205/1 to S.G.), the Australian National Health and Medical Research Council Project grants (GNT1067241 and GNT1139313 to J.J.T) and the Autonomous Province of Trento (Axonomix to G.V and M.M.). Sequencing of Hfq CLASH data was carried out by Edinburgh Genomics that is partly supported through core grants from NERC (R8/H10/56), MRC (MR/K001744/1) and BBSRC (BB/J004243/1).

\section{Competing interest.}

1289 The authors declare no competing financial interest.

\section{Materials \& Correspondence}

All requests for code, materials and reagents should be sent to Sander Granneman

(sgrannem@ed.ac.uk)

\section{References}

Alba BM, Gross CA. 2004. Regulation of the Escherichia coli ??E-dependent envelope stress response. Mol Microbiol 52:613-619. doi:10.1111/j.1365-2958.2003.03982.x 
MG1655 on LB medium: Monitoring utilization of amino acids, peptides, and nucleotides with transcriptional microarrays. Appl Microbiol Biotechnol 71:317-322. doi:10.1007/s00253-005-0310-5

Baev M V., Baev D, Radek AJ, Campbell JW. 2006b. Growth of Escherichia coli MG1655 on LB medium: Determining metabolic strategy with transcriptional microarrays. Appl Microbiol Biotechnol 71:323-328. doi:10.1007/s00253-006-0392-8

Balleza E, López-Bojorquez LN, Martínez-Antonio A, Resendis-Antonio O, Lozada-Chávez I, Balderas-Martínez YI, Encarnación S, Collado-Vides J. 2009. Regulation by transcription factors in bacteria: Beyond description. FEMS Microbiol Rev 33:133-151. doi:10.1111/j.1574-6976.2008.00145.x

Bandyra KJ, Said N, Pfeiffer V, Górna MW, Vogel J, Luisi BF. 2012. The Seed Region of a Small RNA Drives the Controlled Destruction of the Target mRNA by the Endoribonuclease RNase E. Mol Cell 47:943-953. doi:10.1016/j.molcel.2012.07.015

Beisel CL, Storz G. 2011. The Base-Pairing RNA Spot 42 Participates in a Multioutput Feedforward Loop to Help Enact Catabolite Repression in Escherichia coli. Mol Cell 41:286-297. doi:10.1016/j.molcel.2010.12.027

Bernabò P, Tebaldi T, Groen EJN, Lane FM, Perenthaler E, Mattedi F, Newbery HJ, Zhou H, Zuccotti P, Potrich V, Shorrock HK, Muntoni F, Quattrone A, Gillingwater TH, Viero G. 2017. In Vivo Translatome Profiling in Spinal Muscular Atrophy Reveals a Role for SMN Protein in Ribosome Biology. Cell Rep. doi:10.1016/j.celrep.2017.10.010

Boos W, Shuman H. 1998. Maltose / Maltodextrin System of Escherichia coli : Transport, Metabolism, and Regulation Maltose / Maltodextrin System of Escherichia coli : Transport, Metabolism , and Regulation. Microbiol Mol Biol Rev 62:204-229.

Bossi L, Figueroa-Bossi N. 2007. A small RNA downregulates LamB maltoporin in Salmonella. Mol Microbiol 65:799-810. doi:10.1111/j.1365-2958.2007.05829.x

Bossi L, Maloriol D, Figueroa-Bossi N. 2008. Porin biogenesis activates the $\sigma E$ response in Salmonella hfq mutants. Biochimie 90:1539-1544. doi:10.1016/j.biochi.2008.06.001

Bouvier M, Sharma CM, Mika F, Nierhaus KH, Vogel J. 2008. Small RNA binding to 5' mRNA coding region inhibits translational initiation. Mol Cell 32:827-837.

Bruce HA, Du D, Matak-Vinkovic D, Bandyra KJ, Broadhurst RW, Martin E, Sobott F, Shkumatov A V., Luisi BF. 2018. Analysis of the natively unstructured RNA/proteinrecognition core in the Escherichia coli RNA degradosome and its interactions with regulatory RNA/Hfq complexes. Nucleic Acids Res. doi:10.1093/nar/gkx1083

Chao Y, Li L, Girodat D, Forstner KU, Said N, Corcoran C, Smiga M, Papenfort K, Reinhardt $\mathrm{R}$, Wieden HJ, Luisi BF, Vogel J. 2017. in vivo cleavage map illuminates the central cole of RNase $\mathrm{E}$ in coding and non-coding RNA pathways. Mol Cell 65:39-51. doi:10.1016/j.molcel.2016.11.002

Chao Y, Papenfort K, Reinhardt R, Sharma CM, Vogel J. 2012. An atlas of Hfq-bound transcripts reveals 3' UTRs as a genomic reservoir of regulatory small RNAs. EMBO J 31:4005-4019.

Chao Y, Vogel J. 2016. A 3' UTR-Derived Small RNA Provides the Regulatory Noncoding Arm of the Inner Membrane Stress Response. Mol Cell 61:352-363. doi:10.1016/j.molcel.2015.12.023

Chen S, Zhang A, Blyn LB, Storz G. 2004. MicC, a second small-RNA regulator of omp protein expression in Escherichia coli. J Bacteriol. doi:10.1128/JB.186.20.66896697.2004

Corcoran CP, Podkaminski D, Papenfort K, Urban JH, Hinton JCD, Vogel J. 2012. Superfolder GFP reporters validate diverse new mRNA targets of the classic porin regulator, MicF RNA. Mol Microbiol 84:428-445.

Datsenko KA, Wanner BL. 2000. One-step inactivation of chromosomal genes in Escherichia coli K-12 using PCR products. Proc Natl Acad Sci U S A 97:6640-5. doi:10.1073/pnas.120163297

Davis BM, Waldor MK. 2007. RNase E-dependent processing stabilizes MicX, a Vibrio cholerae sRNA. Mol Microbiol. doi:10.1111/j.1365-2958.2007.05796.x

De Las Peñas A, Connolly L, Gross CA. 1997. The sigmaE-mediated response to 
extracytoplasmic stress in Escherichia coli is transduced by RseA and RseB, two negative regulators of sigmaE. Mol Microbiol 24:373-385. doi:9159523

De Lay N, Gottesman S. 2009. The crp-activated small noncoding regulatory RNA CyaR (RyeE) links nutritional status to group behavior. J Bacteriol. doi:10.1128/JB.01157-08

Feng L, Rutherford ST, Papenfort K, Bagert JD, Van Kessel JC, Tirrell DA, Wingreen NS, Bassler BL. 2015. A Qrr noncoding RNA deploys four different regulatory mechanisms to optimize quorum-sensing dynamics. Cell. doi:10.1016/j.cell.2014.11.051

Gogol EB, Rhodius VA, Papenfort K, Vogel J, Gross CA. 2011. Small RNAs endow a transcriptional activator with essential repressor functions for single-tier control of a global stress regulon. Proc Natl Acad Sci U S A 108:12875-12880.

Granneman S, Kudla G, Petfalski E, Tollervey D. 2009. Identification of protein binding sites on U3 snoRNA and pre-rRNA by UV cross-linking and high-throughput analysis of cDNAs. Proc Natl Acad Sci U S A 106:9613-9618. doi:10.1073/pnas.0901997106

Guo MS, Updegrove TB, Gogol EB, Shabalina SA, Gross CA, Storz G. 2014. MicL, a new

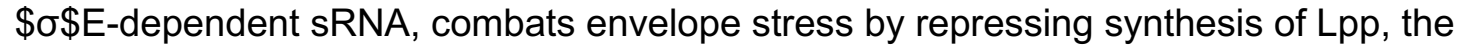
major outer membrane lipoprotein. Genes Dev 28:1620-1634. doi:10.1101/gad.243485.114

Han K, Tjaden B, Lory S. 2016. GRIL-seq provides a method for identifying direct targets of bacterial small regulatory RNA by in vivo proximity ligation. Nat Microbiol 2:16239.

Helwak A, Kudla G, Dudnakova T, Tollervey D. 2013. Mapping the human miRNA interactome by CLASH reveals frequent noncanonical binding. Cell 153:654-665. doi:10.1016/j.cell.2013.03.043

Holmqvist E, Vogel J. 2018. RNA-binding proteins in bacteria. Nat Rev Microbiol. doi:10.1038/s41579-018-0049-5

Holmqvist E, Wagner EGH. 2017. Impact of bacterial sRNAs in stress responses. Biochem Soc Trans. doi:10.1042/BST20160363

Holmqvist E, Wright PR, Li L, Bischler T, Barquist L, Reinhardt R, Backofen R, Vogel J. 2016. Global RNA recognition patterns of post-transcriptional regulators Hfq and CsrA revealed by UV crosslinking in vivo. EMBO J 35:e201593360--1011.

Hör J, Gorski SA, Vogel J. 2018. Bacterial RNA Biology on a Genome Scale. Mol Cell 785799. doi:10.1016/j.molcel.2017.12.023

Hör J, Vogel J. 2017. Global snapshots of bacterial RNA networks. EMBO J 36:245-247. doi:10.15252/embj.201696072

Johansen J, Rasmussen AA, Overgaard M, Valentin-Hansen P. 2006. Conserved small noncoding RNAs that belong to the sigmaE regulon: role in down-regulation of outer membrane proteins. J Mol Biol 364:1-8.

Kenyon WJ, Thomas SM, Johnson E, Pallen MJ, Spector MP. 2005. Shifts from glucose to certain secondary carbon-sources result in activation of the extracytoplasmic function sigma factor \$ $\sigma$ \$E in Salmonella enterica serovar Typhimurium. Microbiology 151:2373-2383. doi:10.1099/mic.0.27649-0

Khemici V, Carpousis AJ. 2004. The RNA degradosome and poly(A) polymerase of Escherichia coli are required in vivo for the degradation of small mRNA decay intermediates containing REP-stabilizers. Mol Microbiol. doi:10.1046/j.13652958.2003.03862.x

Kortmann J, Narberhaus F. 2012. Bacterial RNA thermometers: molecular zippers and switches. Nat Rev Microbiol 10:255-265.

Kudla G, Granneman S, Hahn D, Beggs JD, Tollervey D. 2011. Cross-linking, ligation, and sequencing of hybrids reveals RNA-RNA interactions in yeast. Proc Natl Acad Sci U S A 108:10010-10015. doi:10.1073/pnas.1017386108

Lalaouna D, Carrier M-C, Semsey S, Brouard J-S, Wang J, Wade JT, Massé E. 2015. A 3' External Transcribed Spacer in a tRNA Transcript Acts as a Sponge for Small RNAs to Prevent Transcriptional Noise. Mol Cell 58:393-405.

Lorenz R, Bernhart SH, Höner Zu Siederdissen C, Tafer H, Flamm C, Stadler PF, Hofacker IL. 2011. ViennaRNA Package 2.0. Algorithms Mol Biol 6:26. doi:10.1186/1748-7188-626 
Love MI, Huber W, Anders S. 2014. Moderated estimation of fold change and dispersion for RNA-seq data with DESeq2. Genome Biol 15:550.

Lunelli L, Bernabò P, Bolner A, Vaghi V, Marchioretto M, Viero G. 2016. Peering at Brain Polysomes with Atomic Force Microscopy. J Vis Exp. doi:10.3791/53851

Mäder U, Nicolas P, Depke M, Pané-Farré J, Debarbouille M, van der Kooi-Pol MM, Guérin C, Dérozier S, Hiron A, Jarmer H, Leduc A, Michalik S, Reilman E, Schaffer M, Schmidt F, Bessières P, Noirot P, Hecker M, Msadek T, Völker U, van Dijl JM. 2016. Staphylococcus aureus Transcriptome Architecture: From Laboratory to InfectionMimicking Conditions. PLoS Genet 12:e1005962.

Magoč T, Salzberg SL. 2011. FLASH: Fast length adjustment of short reads to improve genome assemblies. Bioinformatics. doi:10.1093/bioinformatics/btr507

Martínez-Antonio A, Janga SC, Thieffry D. 2008. Functional organisation of Escherichia coli transcriptional regulatory network. J Mol Biol 381:238-247. doi:10.1016/j.jmb.2008.05.054

Melamed S, Faigenbaum-Romm R, Peer A, Reiss N, Shechter O, Bar A, Altuvia Y, Argaman $\mathrm{L}$, Margalit H. 2018. Mapping the small RNA interactome in bacteria using RIL-seq. Nat Protoc 13:1-33.

Melamed S, Peer A, Faigenbaum-Romm R, Gatt YEE, Reiss N, Bar A, Altuvia YYY, Argaman L, Margalit H. 2016. Global Mapping of Small RNA-Target Interactions in Bacteria. Mol Cell 63:884-897. doi:10.1016/j.molcel.2016.07.026

Miyakoshi M, Chao Y, Vogel J. 2015a. Regulatory small RNAs from the 3' regions of bacterial mRNAs. Curr Opin Microbiol. doi:10.1016/j.mib.2015.01.013

Miyakoshi M, Chao Y, Vogel J. 2015b. Cross talk between ABC transporter mRNAs via a target mRNA-derived sponge of the GcvB small RNA. EMBO J 34:e201490546--1492. doi:10.15252/embj.201490546

Monteiro C, Papenfort K, Hentrich K, Ahmad I, Le Guyon S, Reimann R, Grantcharova N, Römling U. 2012. Hfq and Hfq-dependent small RNAs are major contributors to multicellular development in Salmonella enterica serovar typhimurium. RNA Biol. doi:10.4161/rna.19682

Moon K, Gottesman S. 2011. Competition among Hfq-binding small RNAs in Escherichia coli. Mol Microbiol 82:1545-1562. doi:10.1111/j.1365-2958.2011.07907.x

Morita T, Maki K, Aiba H. 2005. RNase E-based ribonucleoprotein complexes: Mechanical basis of mRNA destabilization mediated by bacterial noncoding RNAs. Genes Dev. doi:10.1101/gad.1330405

Mouali Y El, Gaviria-Cantin T, Sá Nchez-Romero MA, Gibert M, Westermann AJ, Rg Vogel J, Balsalobre C. 2018. CRP-cAMP mediates silencing of Salmonella virulence at the post-transcriptional level 1-26. doi:10.1371/journal.pgen.1007401

Navarro Llorens JM, Tormo A, Martínez-García E. 2010. Stationary phase in gram-negative bacteria. FEMS Microbiol Rev 34:476-495. doi:10.1111/j.1574-6976.2010.00213.x

Newbury SF, Smith NH, Higgins CF. 1987. Differential mRNA stability controls relative gene expression within a polycistronic operon. Cell 51:1131-1143. doi:10.1016/00928674(87)90599-X

Nitzan M, Rehani R, Margalit H. 2017. Integration of Bacterial Small RNAs in Regulatory Networks. Annu Rev Biophys 46:131-148. doi:10.1146/annurev-biophys-070816034058

Nues R van, Schweikert G, Leau E de, Selega A, Langford A, Franklin R, losub I, Wadsworth P, Sanguinetti G, Granneman S, Van Nues R, Schweikert G, De Leau E, Selega A, Langford A, Franklin R, losub I, Wadsworth P, Sanguinetti G, Granneman S, Nues R van, Schweikert G, Leau E de, Selega A, Langford A, Franklin R, losub I, Wadsworth P, Sanguinetti G, Granneman S, Van Nues R, Schweikert G, De Leau E, Selega A, Langford A, Franklin R, losub I, Wadsworth P, Sanguinetti G, Granneman S. 2017. Kinetic CRAC uncovers a role for Nab3 in determining gene expression profiles during stress. Nat Commun 8:12. doi:10.1038/s41467-017-00025-5

Papenfort K, Pfeiffer V, Lucchini S, Sonawane A, Hinton JCD, Vogel J. 2008. Systematic deletion of Salmonella small RNA genes identifies CyaR, a conserved CRP-dependent 
riboregulator of OmpX synthesis. Mol Microbiol. doi:10.1111/j.1365-2958.2008.06189.x Papenfort K, Pfeiffer V, Mika F, Lucchini S, Hinton JCD, Vogel J. 2006. oE-dependent small RNAs of Salmonella respond to membrane stress by accelerating global omp mRNA decay. Mol Microbiol 62:1674-1688.

Papenfort K, Said N, Welsink T, Lucchini S, Hinton JCDD, Vogel J. 2009. Specific and pleiotropic patterns of mRNA regulation by ArcZ, a conserved, Hfq-dependent small RNA. Mol Microbiol 74:139-158. doi:10.1111/j.1365-2958.2009.06857.x

Pletnev P, Osterman I, Sergiev P, Bogdanov A, Dontsova O. 2015. Survival guide: Escherichia coli in the stationary phase. Acta Naturae 7:22-33.

Quinlan AR, Hall IM. 2010. BEDTools: a flexible suite of utilities for comparing genomic features. Bioinformatics 26:841-842.

Rhodius VA, Suh WC, Nonaka G, West J, Gross CA. 2006. Conserved and variable functions of the $\sigma E$ stress response in related genomes. PLoS Biol 4:0043-0059. doi:10.1371/journal.pbio.0040002

Sætrom P, Sneve R, Kristiansen KI, Snøve O, Grünfeld T, Rognes T, Seeberg E. 2005. Predicting non-coding RNA genes in Escherichia coli with boosted genetic programming. Nucleic Acids Res. doi:10.1093/nar/gki644

Sedlyarova N, Shamovsky I, Bharati BK, Epshtein V, Chen J, Gottesman S, Schroeder R, Nudler E. 2016. sRNA-Mediated Control of Transcription Termination in E. coli. Cell 167:111--121.e13.

Sezonov G, Joseleau-Petit D, D’Ari R. 2007. Escherichia coli physiology in Luria-Bertani broth. J Bacteriol 189:8746-8749. doi:10.1128/JB.01368-07

Sharma E, Sterne-Weiler T, O'Hanlon D, Blencowe BJ. 2016. Global Mapping of Human RNA-RNA Interactions. Mol Cell 62:618-626. doi:10.1016/j.molcel.2016.04.030

Shimoni Y, Friedlander G, Hetzroni G, Niv G, Altuvia S, Biham O, Margalit H. 2007. Regulation of gene expression by small non-coding RNAs: a quantitative view. Mol Syst Biol 3:138.

Sittka A, Pfeiffer V, Tedin K, Vogel J. 2007. The RNA chaperone Hfq is essential for the virulence of Salmonella typhimurium. Mol Microbiol. doi:10.1111/j.13652958.2006.05489.x

Smirnov A, Förstner KU, Holmqvist E, Otto A, Günster R, Becher D, Reinhardt R, Vogel J. 2016. Grad-seq guides the discovery of ProQ as a major small RNA-binding protein. Proc Natl Acad Sci 113:11591-11596. doi:10.1073/pnas.1609981113

Smirnov A, Wang C, Drewry LL, Vogel J. 2017. Molecular mechanism of mRNA repression in trans by a ProQ-dependent small RNA. EMBO J 36:1029-1045. doi:10.15252/embj.201696127

St-Pierre F, Cui L, Priest DG, Endy D, Dodd IB, Shearwin KE. 2013. One-step cloning and chromosomal integration of DNA. ACS Synth Biol. doi:10.1021/sb400021j

Storz G, Vogel J, Wassarman KM. 2011. Regulation by Small RNAs in Bacteria: Expanding Frontiers. Mol Cell 43:880-891. doi:10.1016/j.molcel.2011.08.022

Tebaldi T, Re A, Viero G, Pegoretti I, Passerini A, Blanzieri E, Quattrone A. 2012. Widespread uncoupling between transcriptome and translatome variations after a stimulus in mammalian cells. BMC Genomics. doi:10.1186/1471-2164-13-220

Thomason MK, Bischler T, Eisenbart SK, Förstner KU, Zhang A, Herbig A, Nieselt K, Sharma CM, Storza G. 2015. Global transcriptional start site mapping using differential RNA sequencing reveals novel antisense RNAs in Escherichia coli. J Bacteriol. doi:10.1128/JB.02096-14

Thompson KM, Rhodius VA, Gottesman S. 2007. SigmaE regulates and is regulated by a small RNA in Escherichia coli. J Bacteriol 189:4243-4256.

Tjaden B. 2015. De novo assembly of bacterial transcriptomes from RNA-seq data. Genome Biol 16:1-10. doi:10.1186/s13059-014-0572-2

Travis AJ, Moody J, Helwak A, Tollervey D, Kudla G. 2013. Hyb: A bioinformatics pipeline for the analysis of CLASH (crosslinking, ligation and sequencing of hybrids) data. Methods.

Tree Jai J., Granneman S, McAteer SP, Tollervey D, Gally DL. 2014. Identification of 
bacteriophage-encoded anti-sRNAs in pathogenic Escherichia coli. Mol Cell 55:199_ 213. doi:10.1016/j.molcel.2014.05.006

Tree J J, Granneman S, McAteer SP, Tollervey D, Gally DL. 2014. Identification of bacteriophage-encoded anti-sRNAs in pathogenic Escherichia coli. doi:10.1016/j.molcel.2014.05.006

Udekwu KI, Wagner EGH. 2007. Sigma E controls biogenesis of the antisense RNA MicA. Nucleic Acids Res. doi:10.1093/nar/gkl1154

Updegrove TB, Zhang A, Storz G. 2016. Hfq: The flexible RNA matchmaker. Curr Opin Microbiol 30:133-138. doi:10.1016/j.mib.2016.02.003

Urban JH, Vogel J. 2007. Translational control and target recognition by Escherichia coli small RNAs in vivo. Nucleic Acids Res 35:1018-1037. doi:10.1093/nar/gkl1040

Vogel J, Luisi BF. 2011. Hfq and its constellation of RNA. Nat Rev Microbiol 9:578-589.

Wassarman KM, Repoila F, Rosenow C, Storz G, Gottesman S. 2001. Identification of novel small RNAs using comparative genomics and microarrays. Genes Dev. doi:10.1101/gad.901001

Waterhouse AM, Procter JB, Martin DMA, Clamp M, Barton GJ. 2009. Jalview Version 2-A multiple sequence alignment editor and analysis workbench. Bioinformatics 25:11891191. doi:10.1093/bioinformatics/btp033

Waters LS, Storz G. 2009. Regulatory RNAs in Bacteria. Cell 136:615-628. doi:10.1016/j.cell.2009.01.043

Waters SA, McAteer SP, Kudla G, Pang I, Deshpande NP, Amos TG, Leong KW, Wilkins MR, Strugnell R, Gally DL, Tollervey D, Tree JJ. 2016. Small RNA interactome of pathogenic E. coli revealed through crosslinking of RNase E. EMBO J 36:e201694639. doi:10.15252/embj.201694639

Webb S, Hector RD, Kudla G, Granneman S. 2014. PAR-CLIP data indicate that Nrd1Nab3-dependent transcription termination regulates expression of hundreds of protein coding genes in yeast. Genome Biol 15:R8. doi:10.1186/gb-2014-15-1-r8

Wilson KS, Hippel PH Von. 1995. Transcription termination at intrinsic terminators: The role of the RNA hairpin (Escherichia coli/RNA polymerase/rho-independent termination). Biochemistry 92:8793-8797. doi:10.1073/pnas.92.19.8793

Wright PR, Georg J, Mann M, Sorescu DA, Richter AS, Lott S, Kleinkauf R, Hess WR, Backofen R. 2014. CopraRNA and IntaRNA: Predicting small RNA targets, networks and interaction domains. Nucleic Acids Res 42:119-123. doi:10.1093/nar/gku359

Wright PR, Richter AS, Papenfort K, Mann M, Vogel J, Hess WR, Backofen R, Georg J. 2013. Comparative genomics boosts target prediction for bacterial small RNAs. Proc Natl Acad Sci 110:E3487-E3496. doi:10.1073/pnas.1303248110

Wu X, Bartel DP. 2017. KpLogo: Positional k -mer analysis reveals hidden specificity in biological sequences. Nucleic Acids Res. doi:10.1093/nar/gkx323 


\section{Supplementary Figures and legends}

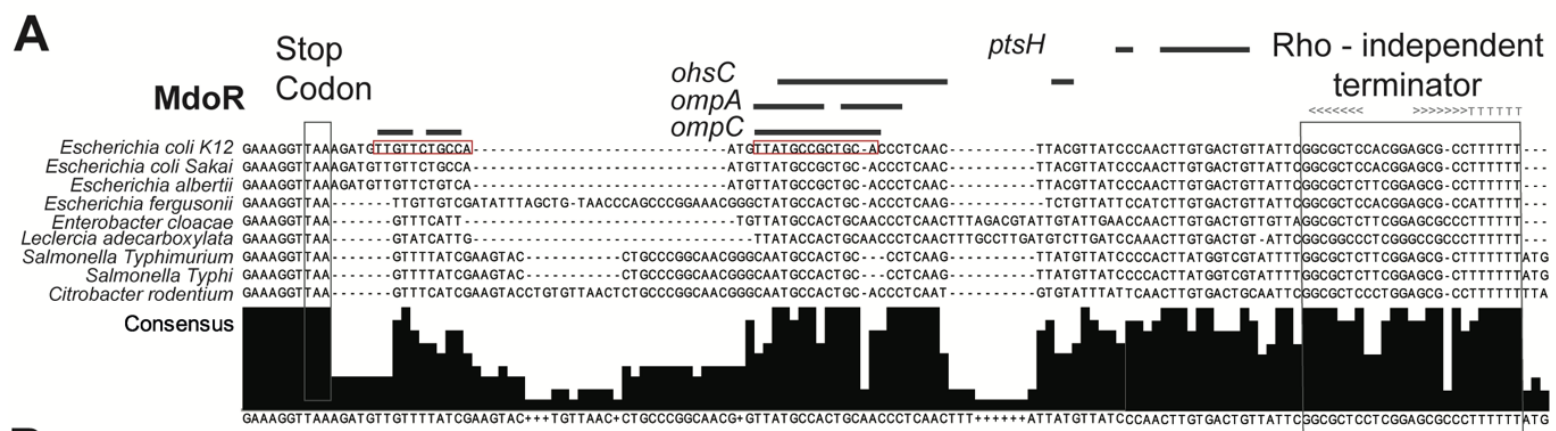

B
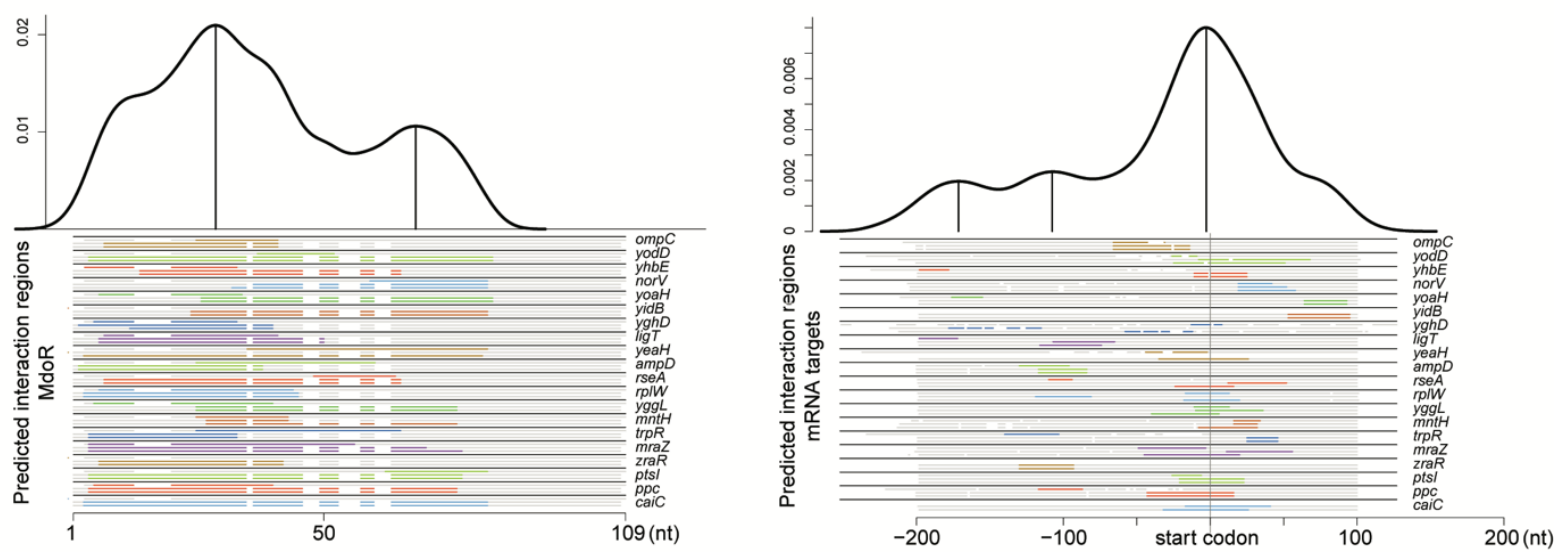

Figure 1-figure supplement 1. Hfq expression and Hfq binding to RNAs at different cell densities in UV-irradiated $E$. coli.

(A) Western blot analyses of Hfq levels during various growth stages. Hfq-HTF was detected using an anti-TAP primary antibody, and a fluorescent secondary antibody. GroEL was used as a loading control.

(B) Quantification of Hfq levels from the Western blot result. The fluorescent signal for HfqHTF and GroEL was measured with the LI-COR from biological replicate experiments. The levels of $\mathrm{Hfq}$ were normalised to GroEL and expressed as fold-change relative to $\mathrm{OD}_{600} 0.4$.

(C) Hfq crosslinking to RNA is similar at each optical density. Autoradiogram showing the purified radioactively labelled $\mathrm{Hfq}-\mathrm{RNA}$ complexes for each $\mathrm{OD}_{600}$ after elution from the nickel beads. Source data for (A-C) are provided as a Source Data file. 
bioRxiv preprint doi: https://doi.org/10.1101/481986; this version posted August 7, 2019. The copyright holder for this preprint (which was

not certified by peer review) is the author/funder, who has granted bioRxiv a license to display the preprint in perpetuity. It is made available under aCC-BY-NC-ND 4.0 International license.
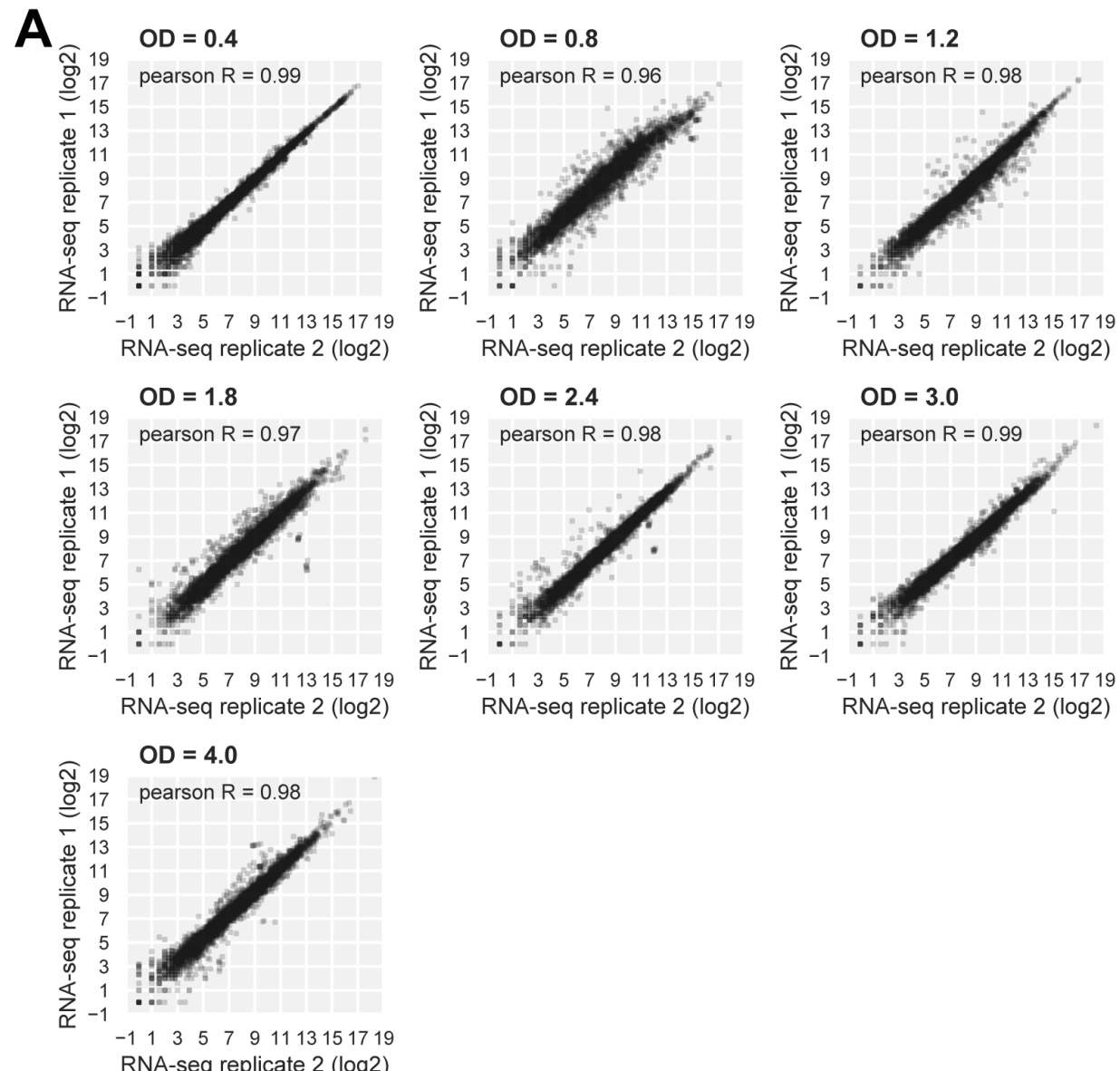

\section{B}
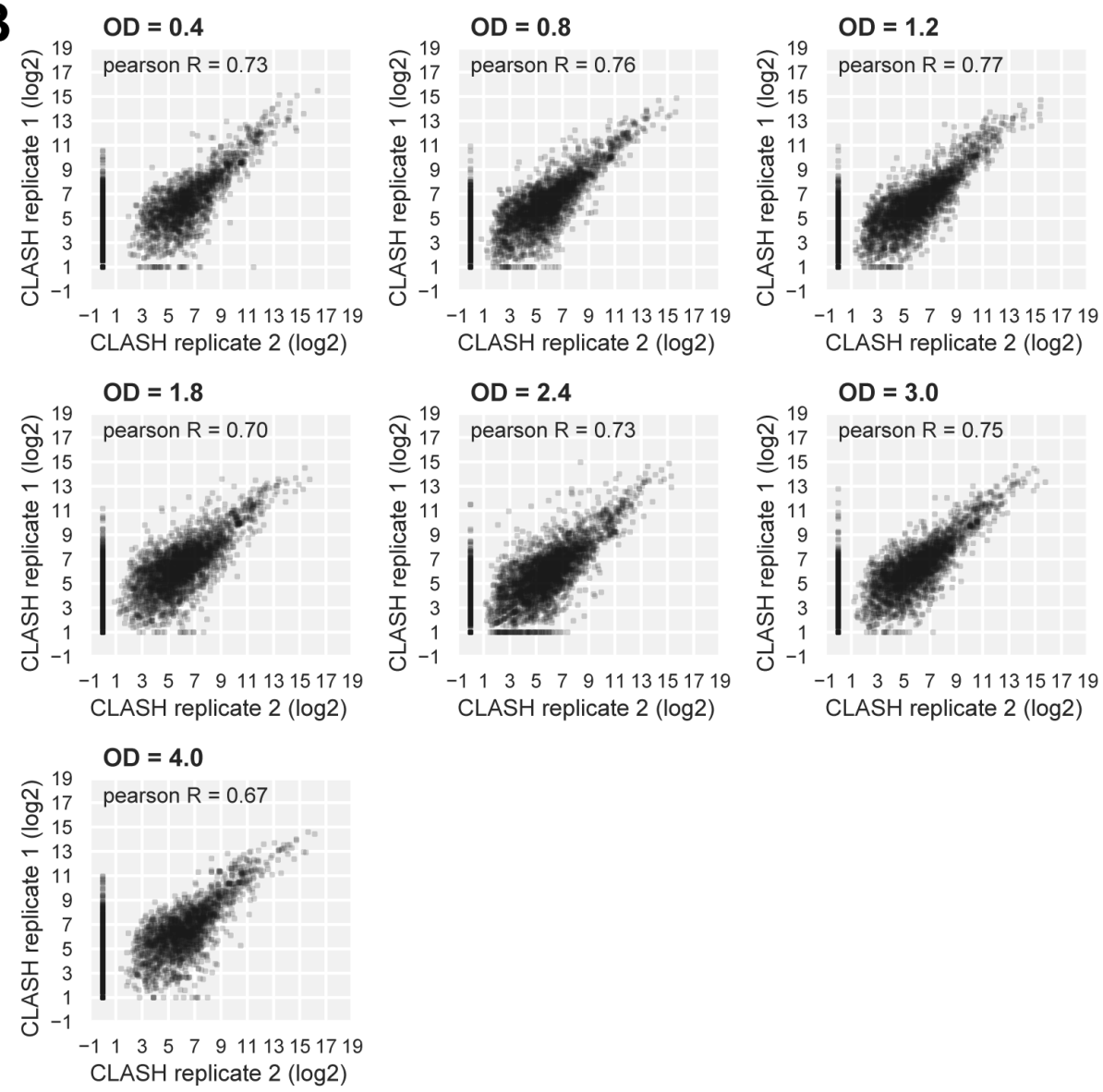
Figure 1-figure supplement 2. RNAseq and Hfq CLASH replicate datasets are highly correlated.

(A, B) Scatter plots showing the distribution of $\log _{2}$ Transcripts Per Million (TPM) normalised read counts for Hfq CLASH (A) and RNA-seq (B) biological replicates. Pearson R coefficients describing the correlation between the two independent experiments at each $\mathrm{OD}_{600}$ are included.

A

B
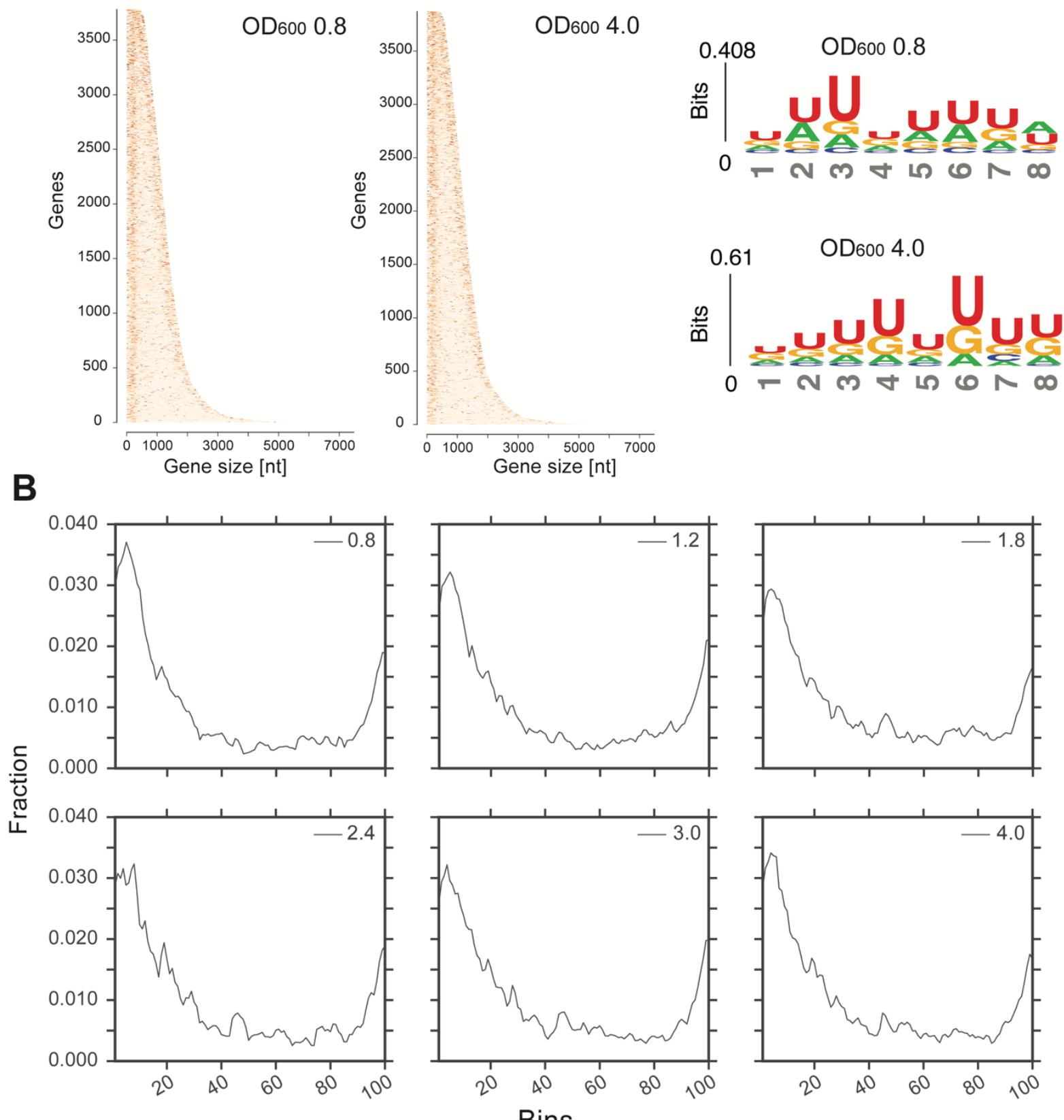

Figure 1-figure supplement 3 . Transcriptome-wide maps of $\mathrm{Hfq}$ binding to mRNA genes.

(A) Heatmaps showing the distribution of $\mathrm{Hfq}$ binding sites across all mRNA genes at $\mathrm{OD}_{600}$ 0.8 and 4.0 . The genes are sorted by their sequence length (x-axis); the darker a nucleotide is, the more $\mathrm{Hfq}$ is crosslinked to it. To generate the heatmap, Hfq binding clusters were generated. A 5'-and 3'UTR length of 200bp was used. 
(B) Hfq binds to poly-U tracks. Significant k-mers (4-8 nt in length) were identified using the pyMotif tool of the pyCRAC package(Webb et al., 2014) and the motif logo was generated using all k-mers with a Z-score > 3, with kpLogo(Wu and Bartel, 2017). (C) A more stringent selection of the genes used to generate the distribution of Hfq binding to the transcriptome: all genes with overlapping 5' or 3'UTRs were removed from the analysis to avoid 'duplicate' counting. For all remaining cDNAs, FDR intervals of minimum $20 \mathrm{nt}$ were considered for distribution plotting. The interval length (with UTR flanks as in the GTF annotation file) for each gene was normalized over 100 bins (x-axis), and the fraction of hits in each bin was calculated (y-axis).

A
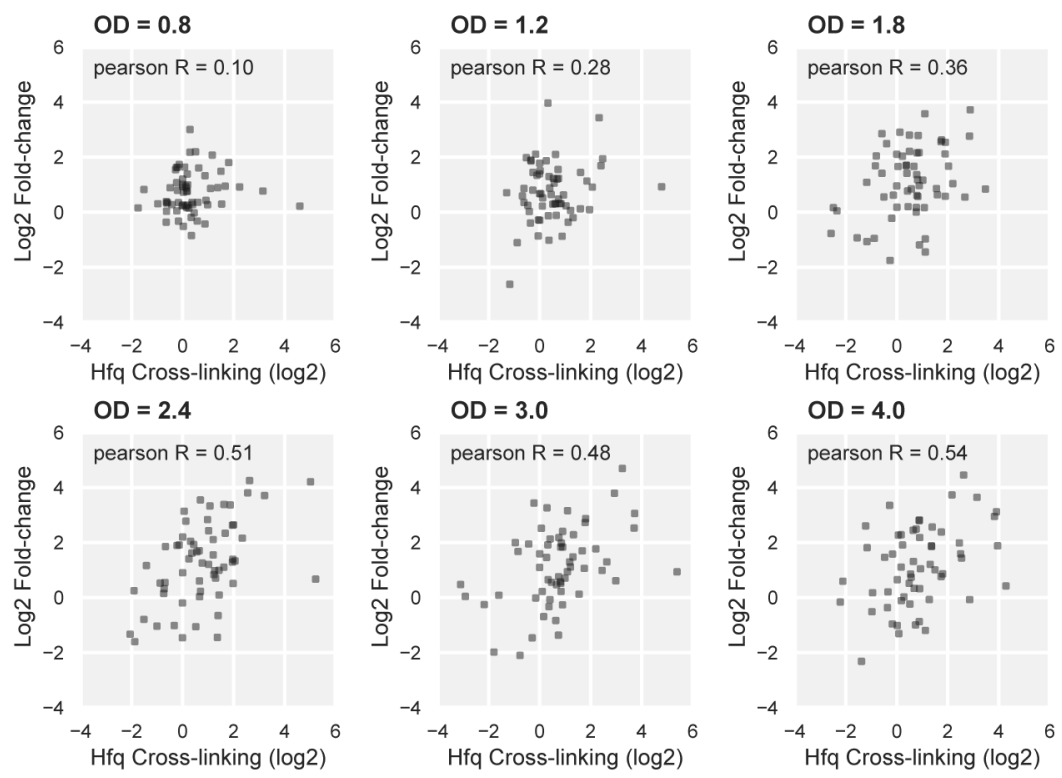

B
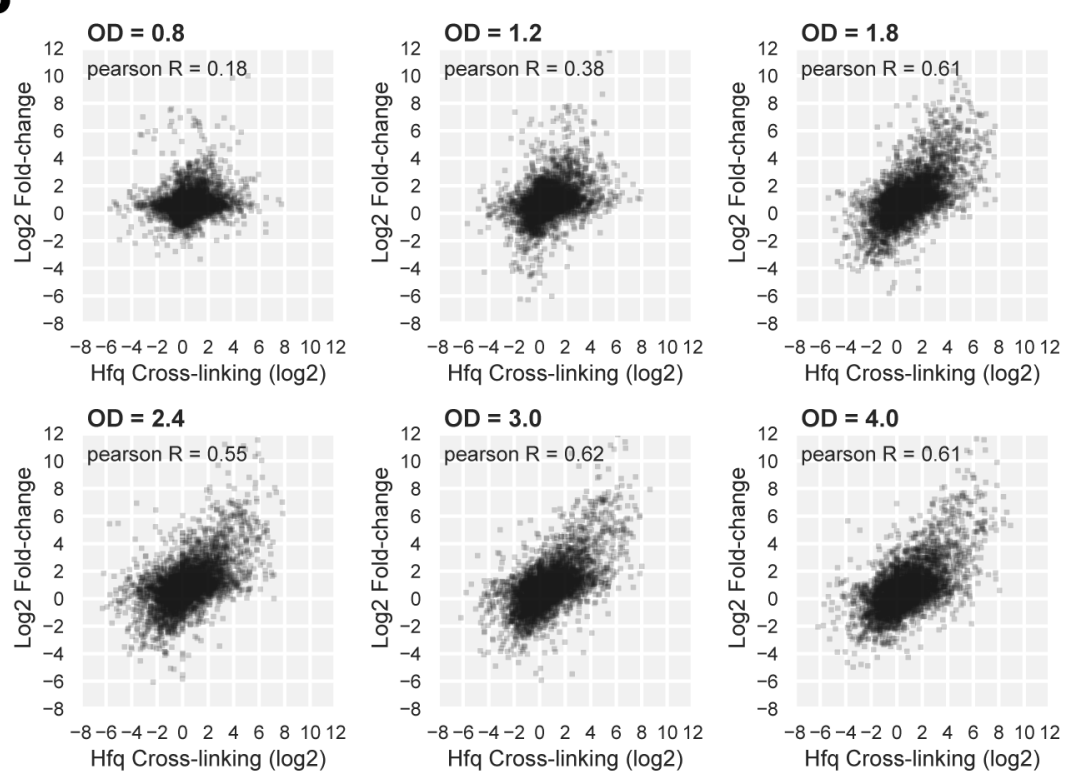

Figure 2-figure supplement 1 . The sRNA and mRNA levels are not always strongly correlated with $\mathrm{Hfq}$ binding during the various stages of growth.

Scatter plots comparing changes in Hfq binding (x-axis) to RNA levels ( $y$-axis) for the indicated $\mathrm{OD}_{600}$ for sRNAs (A) and all transcript classes (B) show that the correlation improves at higher $\mathrm{OD}_{600}$. For each $\mathrm{OD}_{600}$, the Transcripts Per Million (TPM) normalised read counts at each 
bioRxiv preprint doi: https://doi.org/10.1101/481986; this version posted August 7, 2019. The copyright holder for this preprint (which was not certified by peer review) is the author/funder, who has granted bioRxiv a license to display the preprint in perpetuity. It is made available under aCC-BY-NC-ND 4.0 International license.

density-point were divided by the $\mathrm{OD}_{600} 0.4$ data, and the resulting ratios were $\log _{2}$-normalized. Pearson $\mathrm{R}$ coefficients describing the correlation between the two independent experiments at each $\mathrm{OD}_{600}$ is also included for each plot.
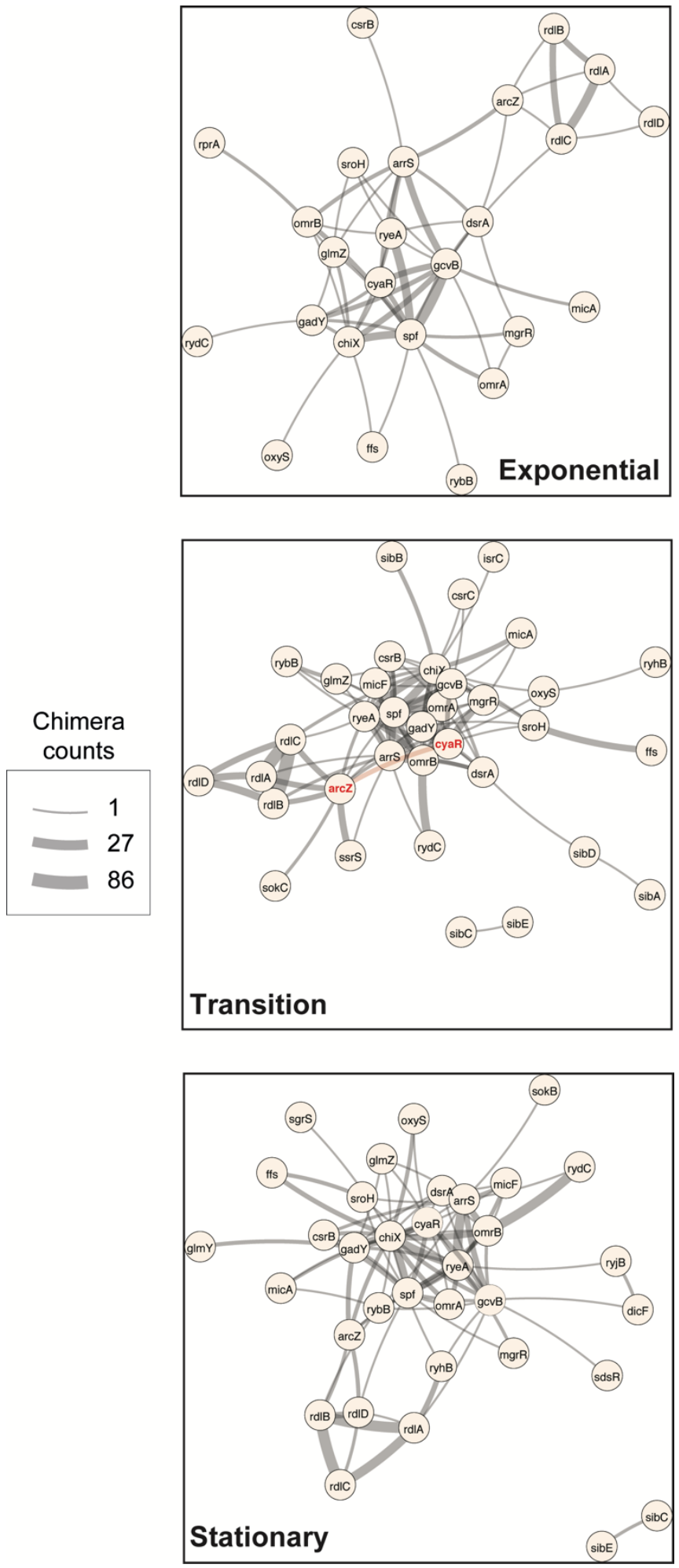

Figure 4-figure supplement 1. sRNA-RNA interactions identified by CLASH are growthstage specific. sRNA-SRNA network generated from the statistically significant CLASH 
bioRxiv preprint doi: https://doi.org/10.1101/481986; this version posted August 7, 2019. The copyright holder for this preprint (which was not certified by peer review) is the author/funder, who has granted bioRxiv a license to display the preprint in perpetuity. It is made available under aCC-BY-NC-ND 4.0 International license.

interactions from two biological replicates, recovered at three main growth stages: exponential $\left(\mathrm{OD}_{600} 0.4\right.$ and 0.8$)$, transition $\left(\mathrm{OD}_{600} 1.2,1.8,2.4\right)$ and early stationary $\left(\mathrm{OD}_{600} 3.0\right.$ and 4.0$)$. The thickness of the edges is proportional to the $\log _{2}$ (unique chimera count for each interaction). Only sRNAs transcribed from independent promoters were included in the analysis.
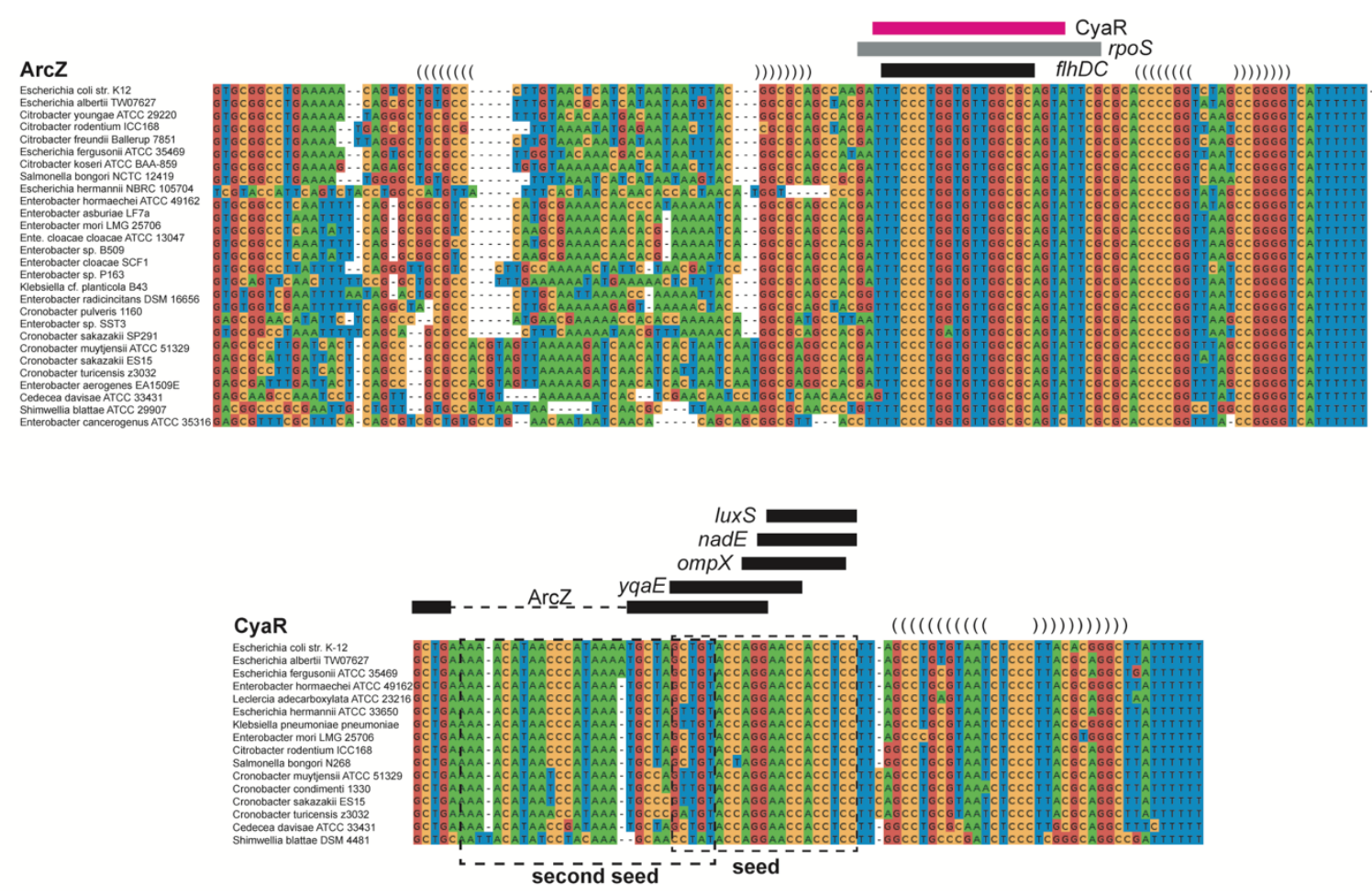

Figure 4-figure supplement 2. Interactions between ArcZ, CyaR and GcvB are conserved. Alignments of ArcZ, CyaR and GcvB were compiled as previously described(van Nues et al., 2016). Names of the enteric bacteria from which the sequence was retrieved are given on the left. Indicated are possible stem-loops (brackets), seed regions (boxed in dashed lines) and their interactions with various sections of ArcZ, CyaR or GcvB (blue and purple bars) or with other sRNAs and mRNAs (black bars). The CyaR sequence indicated with a blue bar is predicted to interact with two regions in GcvB (see blue bars in GcvB alignment), including the second seed sequence. A second interaction (pink bars) involves the seed sequence regions of CyaR and GcvB. 
A
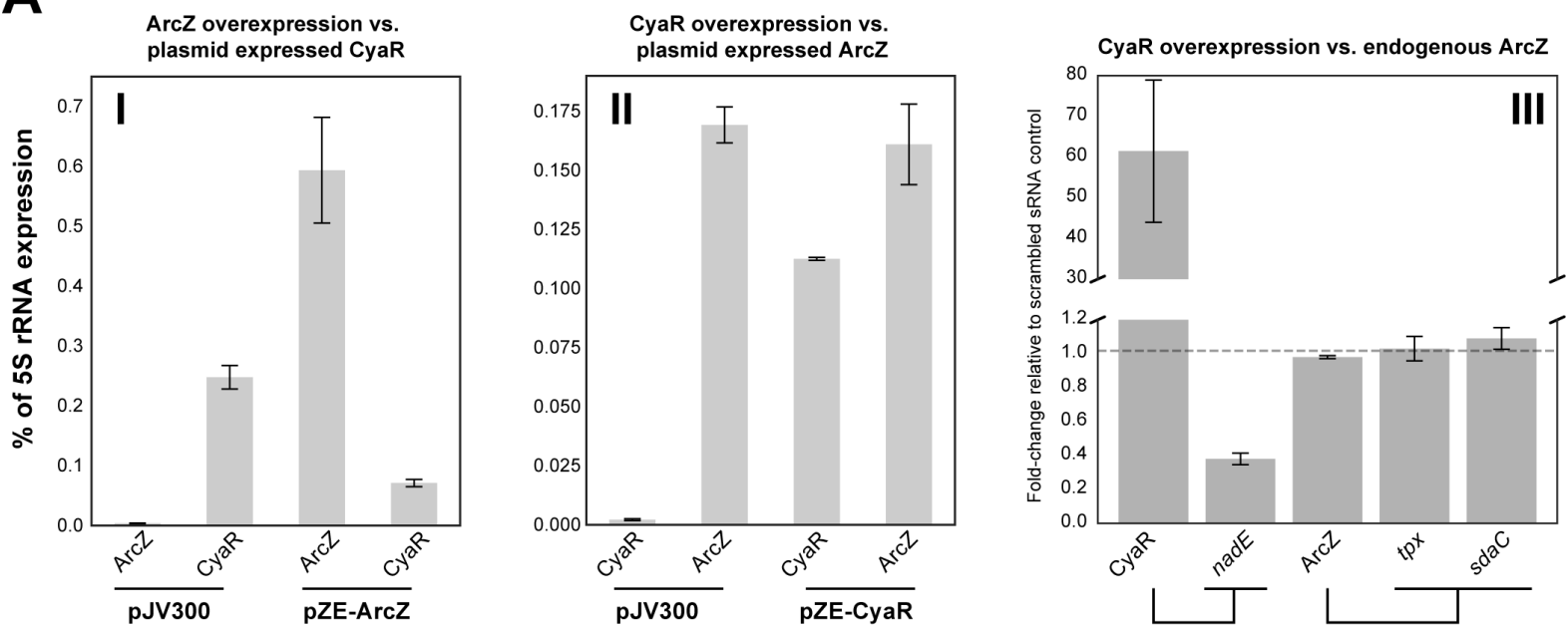

B

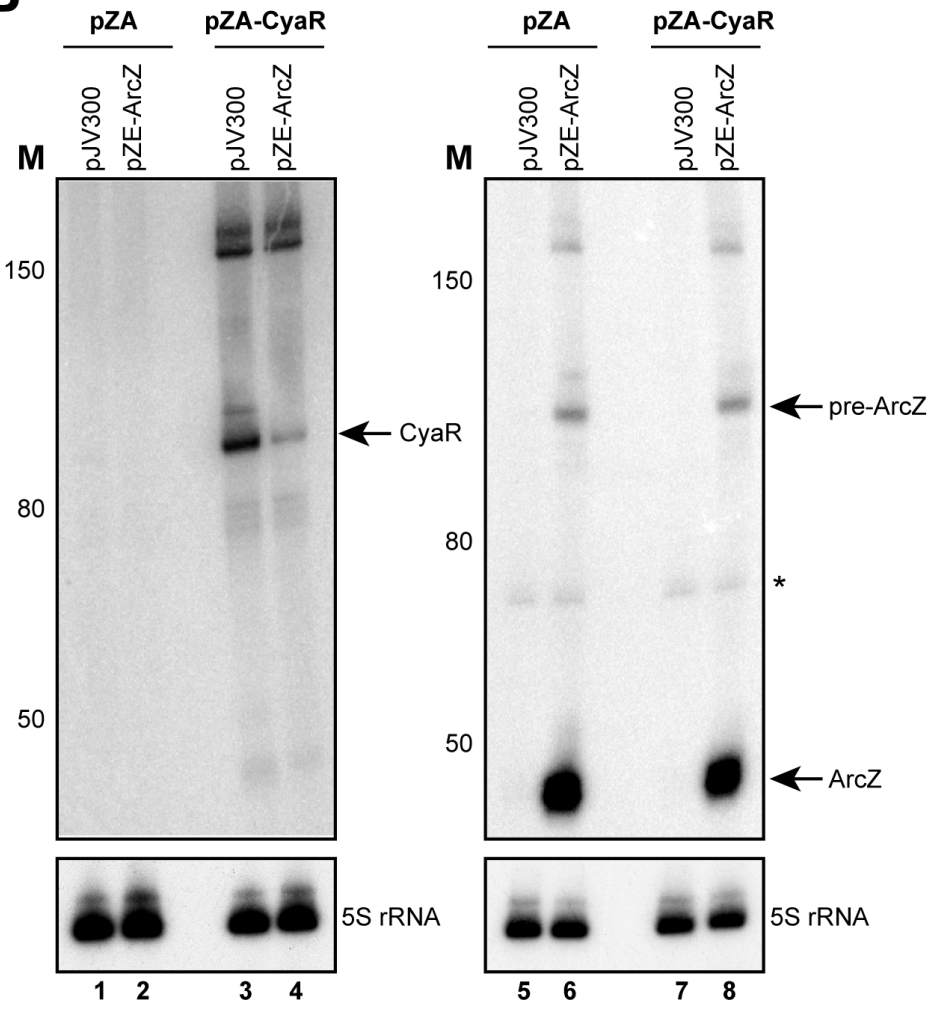

Figure 4-figure supplement 3. ArcZ downregulates CyaR expression.

(A) ArcZ and CyaR were overexpressed from a plasmid-borne IPTG inducible promoter (pZEArcZ and pZE-CyaR) and the data were compared to data from cells carrying a scrambled RNA plasmid (pJV300). The co-expressed candidate target sRNAs (expressed from pZAderived backbone) were induced with anhydrotetracycline hydrochloride (panels I and II). The bars indicate the mean fold-change in expression relative to the level of $5 \mathrm{~S}$ rRNA ( $r r f D)$ in cells with the indicated vector. In panel III endogenous ArcZ levels were measured upon overexpression of CyaR. Error bars indicate the standard error of the mean from three biological replicates and three technical replicates per experiment.

(B) Northern blot analysis of ArcZ and CyaR. The cells containing both the empty pZA and pJV300 plasmids (lanes 1, 5, 9) do not express ArcZ and CyaR at detectable levels. 
A

$$
\mathrm{OD}_{600}
$$

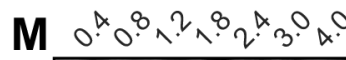

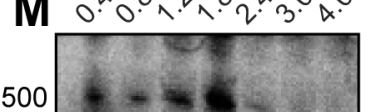

500

300

80

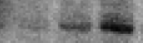

50

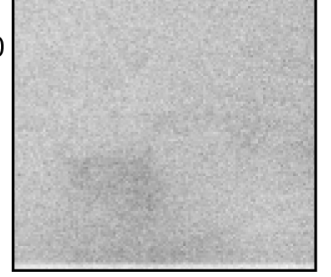

B

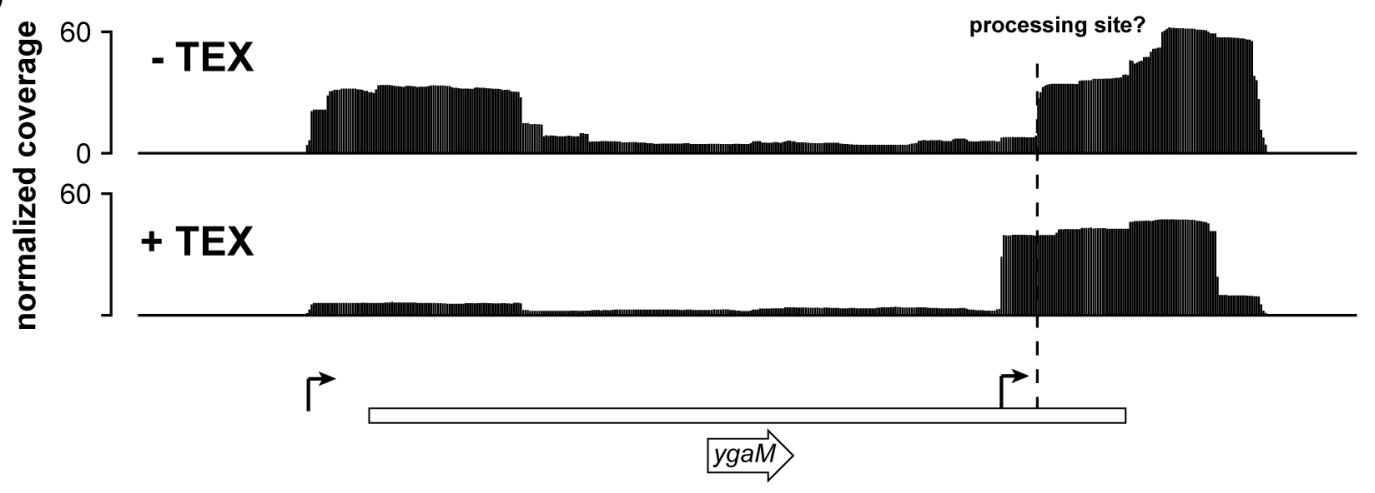

$O D_{600}$

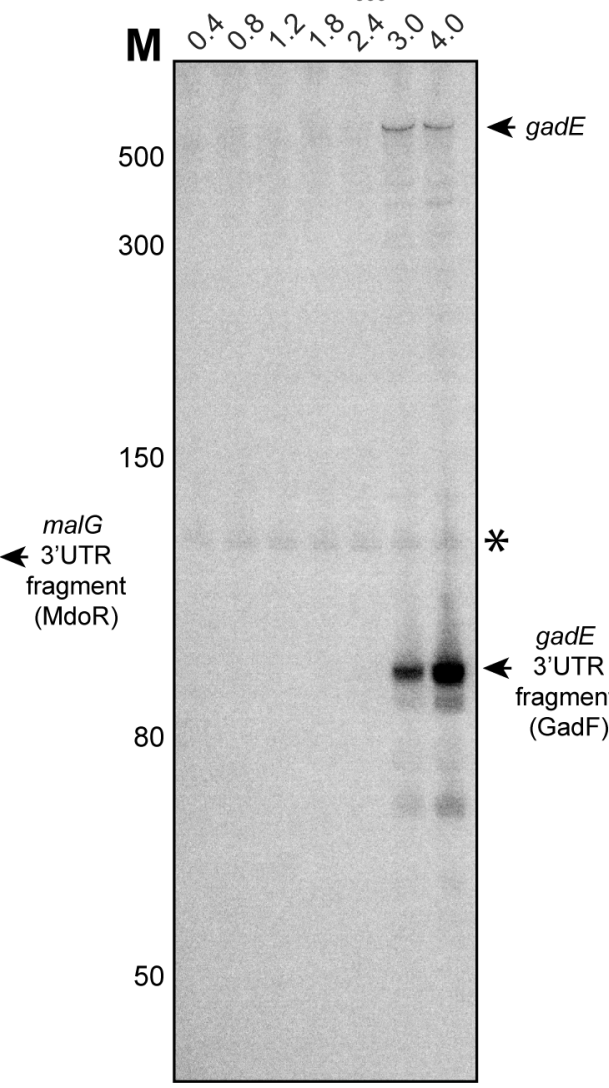

$O D_{600}$

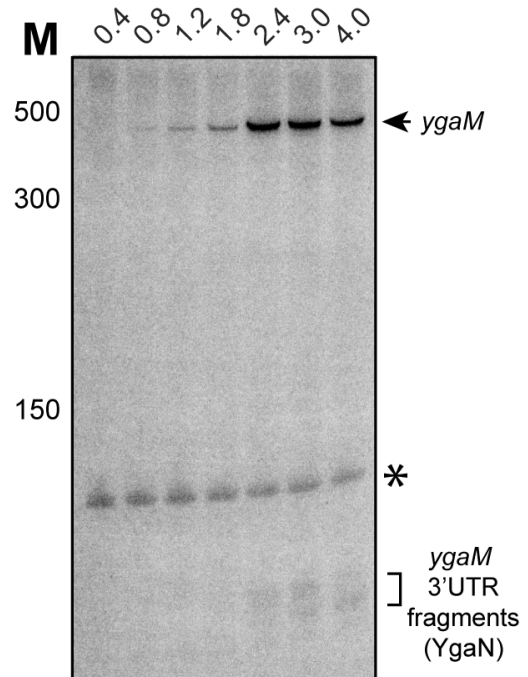

50

80

$(\mathrm{YgaN})$

C

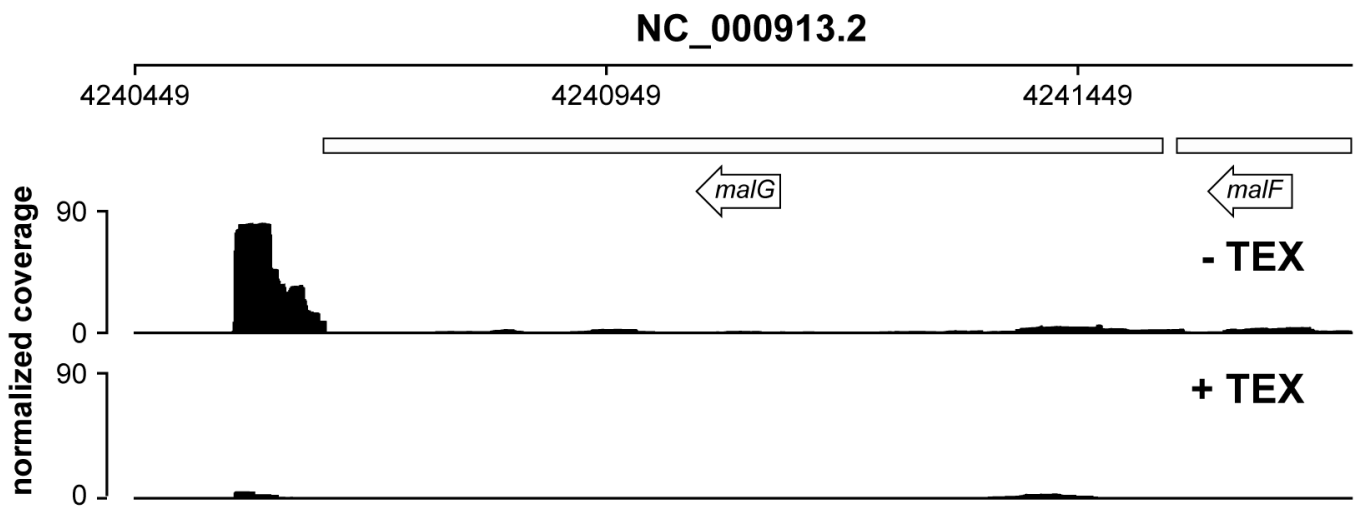


bioRxiv preprint doi: https://doi.org/10.1101/481986; this version posted August 7, 2019. The copyright holder for this preprint (which was not certified by peer review) is the author/funder, who has granted bioRxiv a license to display the preprint in perpetuity. It is made available under aCC-BY-NC-ND 4.0 International license.

Figure 5-figure supplement 1. YgaM, gadE and malG contain sRNAs in their 3'UTRs. (A) Validation of malG 3'UTR (MdoR), ygaM 3'UTR (YgaN) and gadE 3'UTR (GadF) sRNAs by Northern blot. Total RNA extracted from cells at the indicated optical densities $\left(\mathrm{OD}_{600}\right)$ was resolved on $8 \%$ PAA-UREA gels and subjected to Northern blotting using oligos that hybridize with the 3'UTR of the respective transcripts. The asterisk indicates cross-reactivity of the probe with the 5S rRNA. The locations of the 3'UTR-derived fragments are indicated. MdoR and YgaN are $\sim 110$ nt, whereas GadF fragment is $\sim 90$ nt.

(B, C) Analysis of (Terminator 5'-Phosphate Dependent Exonuclease (TEX) RNA-seq datasets(Thomason et al., 2015) indicates that $\mathrm{YgaN}$ has an independent promoter, while MdoR is a degradation product of the malEFG operon. Genome browser tracks showing the location and normalised reads of $y g a M$ and malG fragments in the absence of TEX (-TEX) and in the presence of TEX (+TEX). The ygaM and putative YgaN promoters are indicated. Independently transcribed YgaN could be further processed by RNases, at the site marked with a dashed vertical line.

A

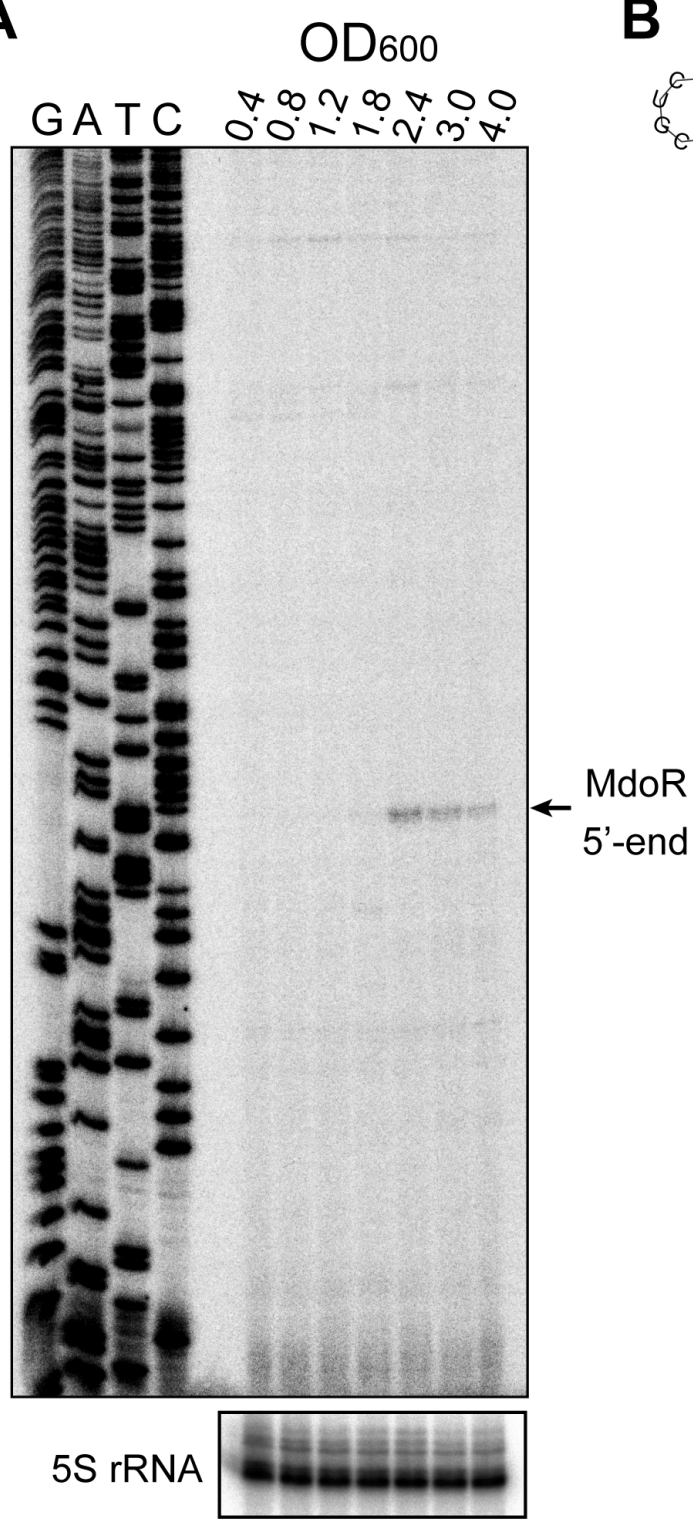

B

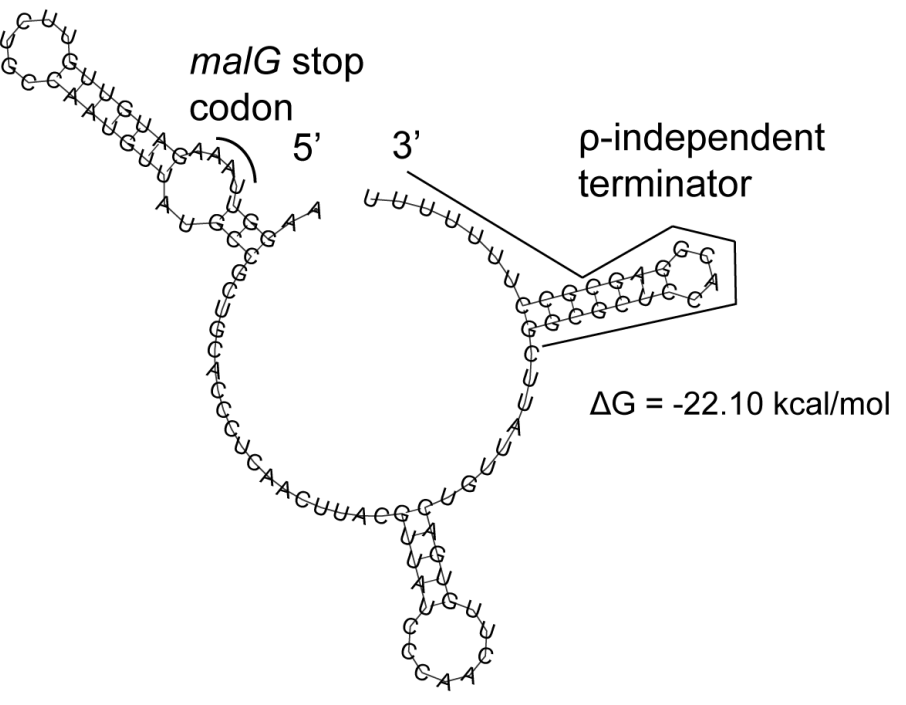


bioRxiv preprint doi: https://doi.org/10.1101/481986; this version posted August 7, 2019. The copyright holder for this preprint (which was not certified by peer review) is the author/funder, who has granted bioRxiv a license to display the preprint in perpetuity. It is made available under aCC-BY-NC-ND 4.0 International license.

Figure 6-figure supplement 1. Conservation and target prediction analyses of MdoR. (A) MdoR contains conserved and variable regions. Sequence conservation analysis of MdoR in several Gram-negative bacteria species (Mafft algorithm with defaults); the arrow indicates the 5'-end of MdoR; the malG stop codon and Rho-independent terminator sites are highlighted; the horizontal black lines indicate the base-pairing regions of MdoR with $p t s H$ (top), OhsC, ompA and ompC (bottom) in E. coli as predicted by CLASH combined with in silico folding (RNACofold).

(B) MdoR is predicted to interact with its targets using two seed regions. Interaction regions within MdoR and top target mRNAs predicted by CopraRNA(Wright et al., 2014, 2013). Density plots showing the relative frequency of a specific MdoR (Left) or mRNA (Right) nucleotide position in all predicted sRNA-mRNA interactions with a p-value $<0.01$ in all considered homologs. The vertical lines indicate local maxima; the aligned regions of the homologs are shown in grey, whereas the interacting regions are shown in arbitrary colors; only the top 20 representative clusters members are shown in the aligned regions, with the gene names indicated on the right. 
A

B
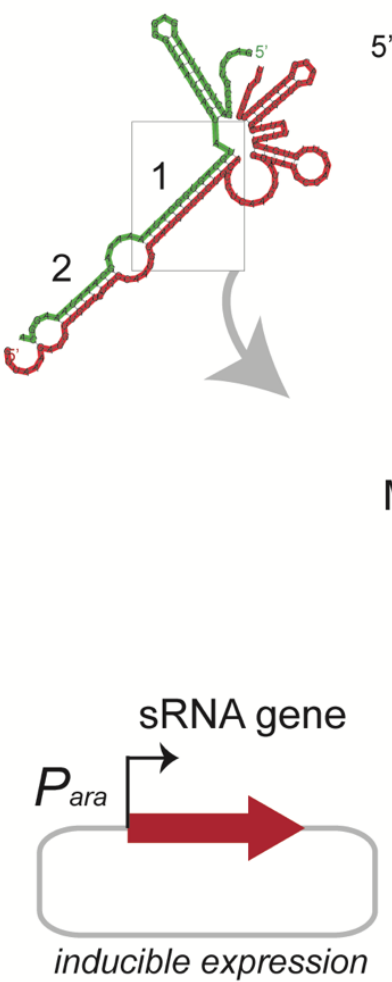

C
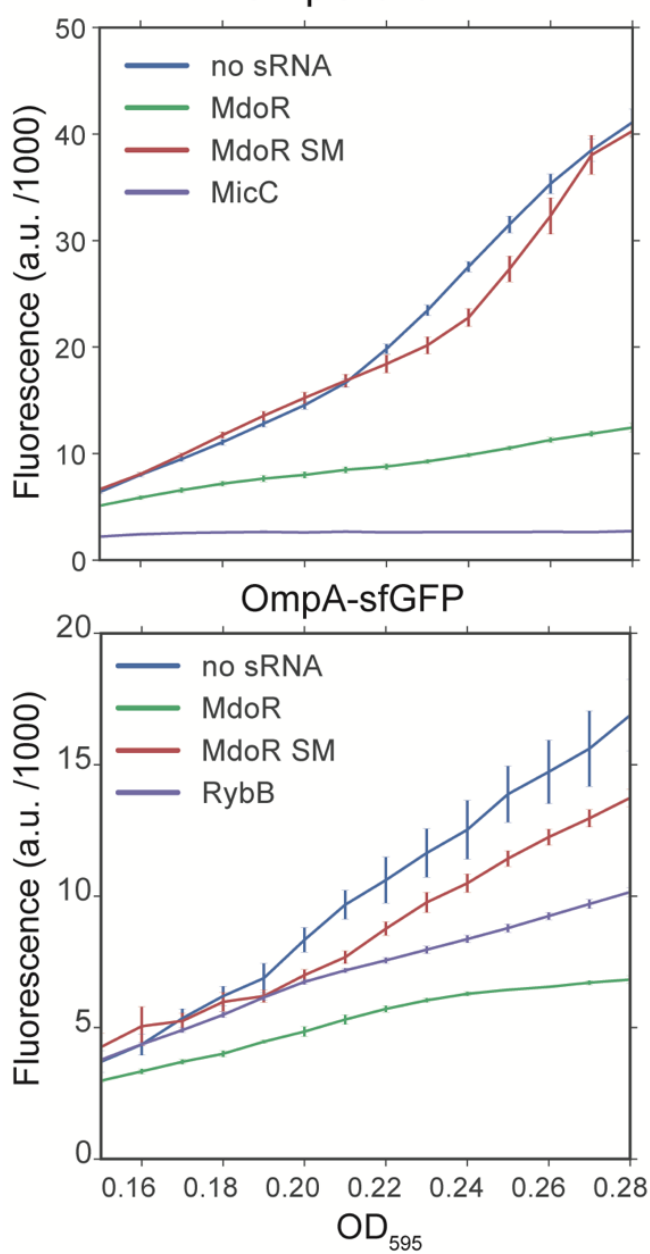

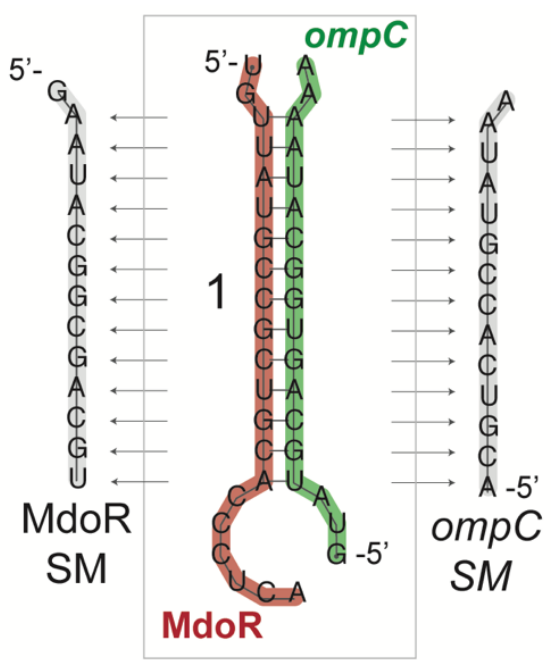

ompC/ompC SM 5'-UTR

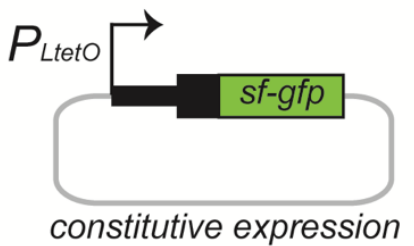

constitutive expression

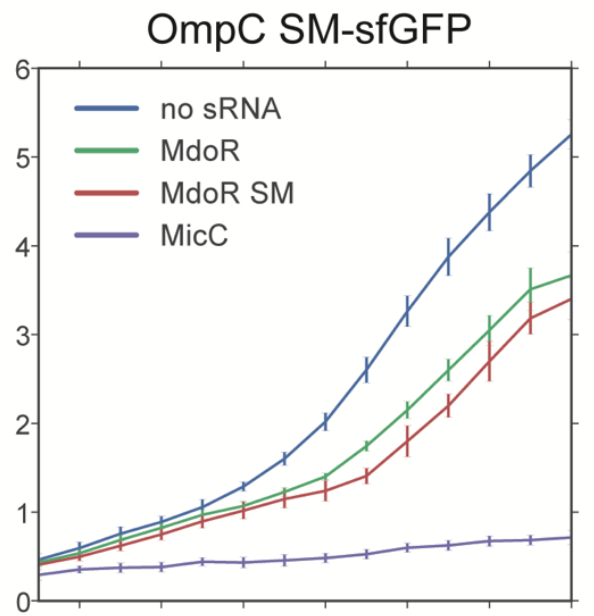

Full-length GFP

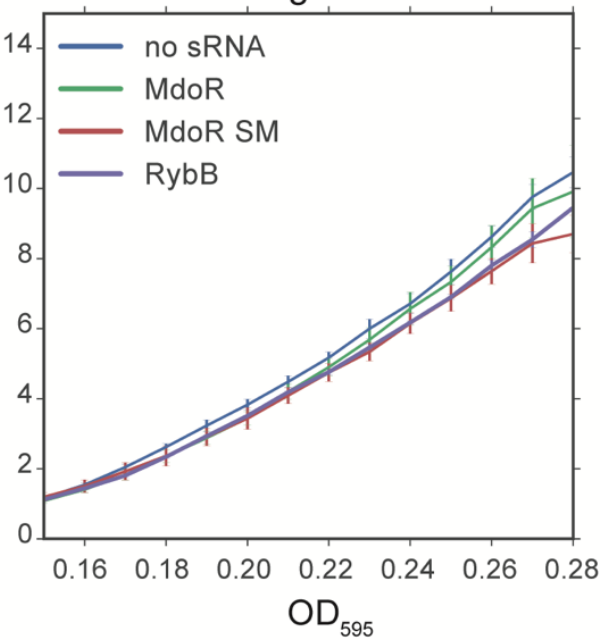


Figure 8-figure supplement 1. Validation of MdoR-ompC interaction using GFP reporters.

(A) Design of the wild-type and mutant $o m p C$ constructs. The panel indicates the base-pairing region within the MdoR-ompC duplex that was mutated. We created an MdoR seed mutant (SM) and an ompC mutant in which base pairing with MdoR SM was restored.

(B) Plasmid system used for the reporter assay: in E. coli TOP10 cells, low-copy plasmids constitutively overexpress target 5'UTRs fused to sfGFP and medium-copy plasmids overexpress the full-length $s R N A s$ upon induction with L-arabinose.

(C) MdoR downregulates expression of OmpC and OmpA sfGFP fusions. In vivo fluorescence measurements of OmpC, OmpC SM and OmpA sfGFP fusion proteins was measured using a Tecan plate reader system. As a negative control we included sfGFP alone in the presence or absence of sRNAs. The 'no sRNA' expressing strains contain the empty pBAD plasmid. The $y$-axis indicates fluorescence units (F.U.) reported by the plate reader. Experiments were performed in technical and biological triplicates; the fluorescence means and SEM of three biological replicates are reported. Source data for are provided as a Source Data file.

A

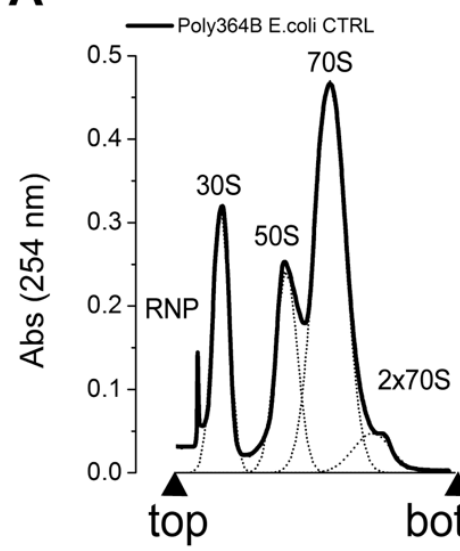

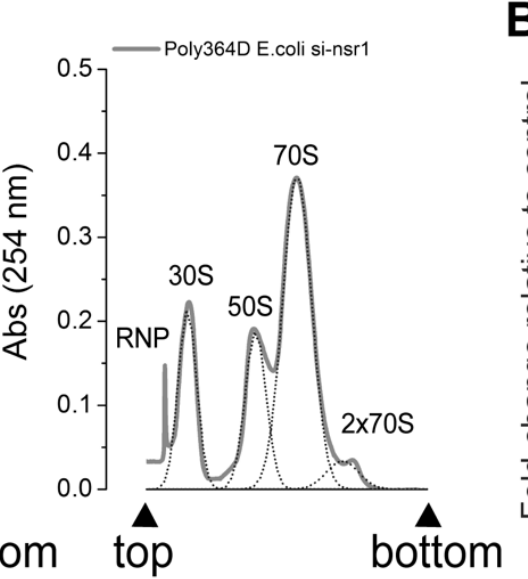

B

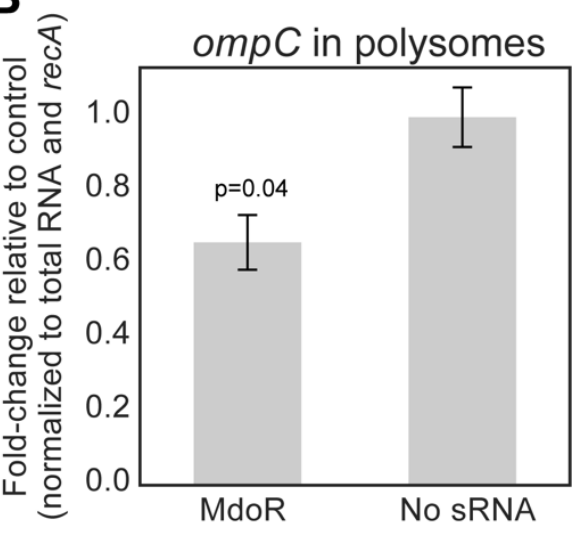

Figure 8-figure supplement 2. MdoR regulates ompC mRNA translation in E. coli.

(A) Cultures at $\mathrm{OD}_{600} 0.4$ overexpressing MdoR or no sRNA for 15 minutes were subjected to polysome profiling. Profiles of the polysomal (2x70S) and subpolysomal fractions obtained for the empty plasmid control and MdoR overexpression samples.

(B) RT-qPCR analysis of the polysomal fractions: A 'total' fraction was obtained by mixing equal amounts/volumes of the polysomal and subpolysomal fractions and is representative of the cytosol/cell lysate content. Total RNA was extracted from all fractions (polysomal, subpolysomal and total). Expression of $o m p C$ in the polysomal fractions was quantified relative to the amount in 'total' fraction, normalized to $\operatorname{rec} A$, and calculated as fold-change relative to the control sample (y-axis). The experiments were performed in technical triplicates; the standard error of the mean (SEM) of three biological replicates fold changes are reported as error bars. Significance of the difference in ompC mRNA level in polysomes was assessed with a two-tailed Student's t-test. Source data for are provided as a Source Data file. 


\section{Supplementary References}

Thomason MK, Bischler T, Eisenbart SK, Förstner KU, Zhang A, Herbig A, Nieselt K, Sharma CM, Storza G. 2015. Global transcriptional start site mapping using differential RNA sequencing reveals novel antisense RNAs in Escherichia coli. $J$ Bacteriol. doi:10.1128/JB.02096-14

van Nues RW, Castro-Roa D, Yuzenkova Y, Zenkin N. 2016. Ribonucleoprotein particles of bacterial small non-coding RNA IsrA (IS61 or McaS) and its interaction with RNA polymerase core may link transcription to mRNA fate. Nucleic Acids Res 44:2577-2592.

Webb S, Hector RD, Kudla G, Granneman S. 2014. PAR-CLIP data indicate that Nrd1-Nab3-dependent transcription termination regulates expression of hundreds of protein coding genes in yeast. Genome Biol 15:R8. doi:10.1186/gb2014-15-1-r8

Wright PR, Georg J, Mann M, Sorescu DA, Richter AS, Lott S, Kleinkauf R, Hess WR, Backofen R. 2014. CopraRNA and IntaRNA: Predicting small RNA targets, networks and interaction domains. Nucleic Acids Res 42:119-123. doi:10.1093/nar/gku359

Wright PR, Richter AS, Papenfort K, Mann M, Vogel J, Hess WR, Backofen R, Georg J. 2013. Comparative genomics boosts target prediction for bacterial small RNAs. Proc Natl Acad Sci 110:E3487-E3496. doi:10.1073/pnas.1303248110

Wu X, Bartel DP. 2017. KpLogo: Positional k -mer analysis reveals hidden specificity in biological sequences. Nucleic Acids Res. doi:10.1093/nar/gkx323 